\title{
ON THE RANK OF THE REES-SUSHKEVICH VARIETIES
}

\author{
S. I. KUBLANOVSKII
}

\begin{abstract}
A specific numerical characteristic of a variety of semigroups, the rank, is introduced. It is proved that the Rees-Sushkevich varieties with the same derivative, i.e., containing the same 0 -simple semigroups, are determined by their rank uniquely up to permutation identities. As a consequence, answers to several well-known questions are obtained. In particular, a description is given for the Rees-Sushkevich varieties satisfying finiteness conditions (finiteness of the base of identities or of the lattice of subvarieties, generation by a finite semigroup or by a completely 0-simple semigroup, the condition of maximality, minimality, finite width, etc.). Some applications of an algorithmic nature are presented. In particular, it is shown that a Rees-Sushkevich variety defined by a finite set of identities or by a finite semigroup has a decidable (polynomially decidable) equational theory if and only if its derivative has the same property. This holds true for combinatorial varieties.
\end{abstract}

\section{§1. INTRODUCTION}

The so-called simple objects play a special role in algebraic theories. In group theory, these are simple groups (i.e., the groups without nontrivial normal subgroups), in the theory of algebras such objects are simple algebras (i.e., the algebras without proper ideals), and in the theory of semigroups, the role of simple objects is played by the 0 -simple semigroups (the semigroups with nonzero square and without proper nonzero ideals). This is explained by the fact that arbitrary algebraic objects can be constructed by successive extensions of simple objects. In 1928, Sushkevich (see [7) obtained a description of finite simple semigroups in terms of some matrices over finite groups. In 1940, Rees [15] extended the Sushkevich result to the case of infinite semigroups, namely, to the 0 -simple semigroups containing a minimal nonzero idempotent. Such semigroups acquired the name of completely 0 -simple semigroups, and their matrix representations became known as Rees matrix semigroups with zero over groups [7]. The Rees theorem is an analog of the Wedderburn-Artin theorem for rings and algebras over fields. This result is important because it turned out that every periodic 0-simple semigroup is completely 0 -simple (the Rees theorem [15, 7]), i.e., such a semigroup admits a Rees matrix representation. This explains the interest in subvarieties of periodic varieties generated by 0 -simple semigroups. Such varieties are called the Rees-Sushkevich varieties. One of the problems concerning these varieties was to find a basis of identities for Rees matrix semigroups over groups of a given exponent $n$ (the variety generated by these semigroups is denoted by $R S_{n}$ ).

This problem was solved in 1997 (see [9]). It was established that the three identities

$\left(\mathrm{I}_{n}\right) x^{2}=x^{n+2}$,

$\left(\mathrm{II}_{n}\right) x y x=(x y)^{n+1} x$,

$\left(\mathrm{III}_{n}\right)(x h z)^{n} \cdot x y z=x y z \cdot(x h z)^{n}$

form a basis of identities in $R S_{n}$.

2010 Mathematics Subject Classification. Primary 20M07.

Key words and phrases. Semigroups, 0-prime, identity, rank, Rees-Sushkevich variety, Cross semigroup, finitely based semigroup, finite generation, smallness, polynomial algorithm. 
Not every subvariety of $R S_{n}$, i.e., not every Rees-Sushkevich variety is generated by completely 0 -simple semigroups. A variety is said to be exact if it is generated by completely 0 -simple semigroups. For every variety $V$ of semigroups, let $V^{\prime}$ denote the variety generated by all completely 0 -simple semigroups in $V$. The variety $V^{\prime}$ is called the derivative of $V$. Thus, a variety $V$ is exact if and only if $V=V^{\prime}$. In 1996, the author obtained necessary conditions for a variety of semigroups to be exact. It turned out that these conditions are not sufficient. Necessary and sufficient conditions for exactness were found by the author and, independently, by N. Reilly [16]. These conditions are similar but differ substantially. Reilly's conditions are stated in terms of identities and indicator systems. The conditions found independently by the author are given in terms of indicator systems only, which makes it possible to recognize algorithmically whether or not a variety given by a finite basis of identities is exact. In the present paper, the author's result is published for the first time with a complete proof. This result is obtained as a consequence (Corollary 1 ) of the main result of the paper. We construct a series of special finite combinatorial semigroups and prove (Theorem 1) that every ReesSushkevich variety is generated by a completely 0-simple semigroup and a collection of such special semigroups. Every collection of special semigroups is determined by a tuple (vector) of 12 nonnegative integer coordinates. This tuple will be called the rank. It turns out (Theorem 5) that a Rees-Sushkevich variety $V$ is determined by its rank and the derivative $V^{\prime}$ uniquely up to permutation identities. We recall that a permutation identity is an identity of the form $x_{1} x_{2} \ldots x_{k}=x_{\sigma 1} x_{\sigma 2} \ldots x_{\sigma k}$, where $\sigma$ is a nontrivial permutation. We also recall that the degree of a variety $V$ is the largest nilpotency degree of the nilpotent semigroups in $V$.

In the present paper, we introduce the notions of radical and semisimple varieties. A variety $V$ is said to be semisimple if none of the permutation identities with length $k$ less than the degree of $V$ are valid in $V$. A variety $V$ is radical if the identities $x^{2}=0$ and $x y x=0$ and a permutation identity of length $k$ less than the degree of $V$ are valid in $V$. One of the main results of the paper is Theorem 3, which says that an arbitrary Rees-Sushkevich variety can be represented as the sum of a radical variety and a semisimple variety. In Part 2 of the present paper, we give applications of these results. In particular, we obtain the following necessary and sufficient condition for a Rees-Sushkevich variety to be finitely based (Corollary 4): a Rees-Sushkevich variety $V$ is finitely based (hereditarily finitely based) if and only if the derivative variety $V^{\prime}$ has the same property. This immediately implies an affirmative answer to the question as to whether the well-known matrix semigroups $A_{2}$ and $B_{2}$ are hereditarily finitely based. This result was obtained recently by E. Lee [10, 11]. The above criterion for a variety to be finitely based was obtained by the author in 2008. Here we give a short proof of this result. The proof is based on the description of the identities of the special combinatorial semigroups (see Theorem 4). Another application of the main results is the description of Rees-Sushkevich varieties satisfying various finiteness conditions. In numerous papers on this topic, the following finiteness conditions appear.

1) Small varieties. A variety $V$ is small if the lattice of the subvarieties of $V$ is finite. The problem of describing the small varieties was first posed by Evans in 1971 and is open up to now (see [1]).

2) Finitely generated varieties. A variety $V$ is finitely generated if $V$ is generated by a finite semigroup. The main open problem here is to find an algorithm that decides whether $V$ is finitely generated if $V$ is given by a finite basis of identities.

3) Cross varieties. A variety $V$ is said to be Cross if $V$ is small, finitely generated, and has a finite basis of identities. The Cross property was first introduced in 1960 by 
Higman for varieties of groups. By a theorem of Oates and Powell [8], a variety generated by a finite group is Cross.

4) Minimality and maximality conditions. We say that a variety $V$ satisfies the minimality (maximality) condition if the lattice of subvarieties of $V$ has no infinite descending (ascending) chains. The problem of describing such varieties is also open in the general case (see [1]).

5) Varieties of finite width. We say that a variety $V$ has finite width if, in the lattice of its subvarieties, the lengths of the antichains are uniformly bounded. In [2, Sapir and Sukhanov prove that if a variety $V$ of semigroups has finite width, then $V$ is a periodic and permutation variety, or a variety of finite degree. The converse is not true in general (see the survey [1]).

In the present paper, we describe the Rees-Sushkevich varieties in the case of each of conditions 1-5 (Corollaries 9 16). In particular, this implies a recent result by Lee [12 concerning the description of small, finitely generated, and Cross combinatorial (i.e., included in $R S_{1}$ ) Rees-Sushkevich varieties. The concluding part of the paper is devoted to algorithmic questions. We prove (Theorem 6 ) that, for an arbitrary variety $V$ defined by a finite number of identities, there is an algorithm of linear complexity (in the sum of lengths of the identities) allowing one to calculate the rank of $V$. Theorem 7 says that a Rees-Sushkevich variety $V$ defined by a finite number of identities or by a finite semigroup has decidable (polynomially decidable) equational theory if and only if the derivative $V^{\prime}$ of $V$ has the same property. This implies that every finite combinatorial Rees-Sushkevich semigroup has a polynomially decidable equational theory. In other words, the verification of an identity in such a semigroup can be performed in a polynomial time (relative to the length of the identity). The results of the papers 18, 3. show that this statement cannot be improved in the sense that, lifting the requirement that subgroups be trivial or that the Rees-Sushkevich identities hold, we can construct counterexamples. We prove (see Theorem 8) that there exists a polynomial complexity algorithm for deciding whether a periodic semigroup variety defined by a finite set of identities or by a finite semigroup is generated by completely 0 -simple semigroups.

The author is deeply grateful to A. V. Yakovlev for suggestions and remarks, which considerably improved the paper and simplified proofs, and to M. V. Volkov for numerous helpful discussions.

\section{§2. Definitions and notation}

1. A letter $x$ of a word $u$ is said to be cyclic if $x$ belongs to a cycle, i.e., to a subword $u^{\prime}$ of length at least 2 beginning and ending with the same letter. We denote by $c(u)$ the set of letters of $u$ and by $h(u)$ and $t(u)$, respectively, the first and the last letter of $u$. The length of $u$ (i.e., the number of letters in it) is denoted by $|u|$.

We recall that a word is said to be covered by cycles if each letter of this word is cyclic. If all letters of a word are distinct, we say that this word is singular. A word containing more than one letter is regular if it cannot be represented as a product of words with disjoint alphabets; such words are also said to be connected.

2. Let $u$ be a nonsingular word. It can uniquely be represented in the form

$$
u=w_{1} u_{1} w_{2} u_{2} \ldots w_{m} u_{m} w_{m+1},
$$

where $u_{1}, u_{2}, \ldots, u_{m}$ are regular words, $w_{1}, w_{2}, \ldots, w_{m+1}$ are singular words (possibly, empty), and distinct factors in this decomposition have no common letters. The number $m$ is called the cyclic characteristic of $u$ and the decomposition itself is called the canonical decomposition of the first kind. 
Similarly, every nonsingular word $u$ can uniquely be represented in the form

$$
u=w_{1} u_{1} w_{2} u_{2} \ldots w_{m} u_{m} w_{m+1},
$$

where $u_{1}, u_{2}, \ldots, u_{m}$ are words covered by cycles, $w_{1}, w_{2}, \ldots, w_{m+1}$ are singular words, the words $w_{2}, \ldots, w_{m}$ are nonempty, and distinct factors have no common letters. The number $m$ is called the quasicyclic characteristic of $u$ and the decomposition itself is called the canonical decomposition of the second kind.

The decompositions of the 1st and 2nd kind of a singular word $u$ coincide with $u$; by definition, the cyclic and the quasicyclic characteristics of a singular word are zero.

3. Two words $u$ and $v$ are similar (respectively, congruent) if their cyclic (respectively, quasicyclic) characteristics are equal and their canonical decompositions of the 1st kind (respectively, of the 2nd kind)

$$
u=w_{1} u_{1} w_{2} u_{2} \ldots w_{m} u_{m} w_{m+1}, \quad v=w_{1} v_{1} w_{2} v_{2} \ldots w_{m} v_{m} w_{m+1}
$$

satisfy $c\left(u_{i}\right)=c\left(v_{i}\right)$ for all $i, 1 \leq i \leq m$.

4. An identity $u=v$ is called an $A_{0}$-identity if the words $u$ and $v$ are similar. An identity $u=v$ is called a $B_{0}$-identity if the words $u$ and $v$ are congruent. A variety of semigroups is called an $A 0-(B 0)$-variety if its identities are $A_{0^{-}}\left(B_{0}\right)$-identities. This is equivalent to the existence of a basis consisting of $A_{0^{-}}\left(B_{0}\right)$-identities.

5. An $A_{0}$-identity

$$
w_{1} u_{1} w_{2} u_{2} \ldots w_{m} u_{m} w_{m+1}=w_{1} v_{1} w_{2} v_{2} \ldots w_{m} v_{m} w_{m+1}
$$

both sides of which are represented by decompositions of the 1st kind is called a $\bar{\lambda}$-identity if $h\left(u_{i}\right) \neq h\left(v_{i}\right)$ for some $i$, and a $\bar{\rho}$-identity if $t\left(u_{i}\right) \neq t\left(v_{i}\right)$ for some $i$.

A $B_{0}$-identity

$$
w_{1} u_{1} w_{2} u_{2} \ldots w_{m} u_{m} w_{m+1}=w_{1} v_{1} w_{2} v_{2} \ldots w_{m} v_{m} w_{m+1}
$$

both sides of which are represented by decompositions of the 2 nd kind is called a $\lambda$-identity if $h\left(u_{i}\right) \neq h\left(v_{i}\right)$ for some $i$, and a $\lambda^{*}$-identity if $h\left(u_{1}\right) \neq h\left(v_{1}\right)$. The definitions of $\rho$ - and $\rho^{*}$-identities are obtained by dualization.

6. A variety $V$ of semigroups is called a $\lambda$-variety if all identities of $V$ are $B_{0}$-identities and one of these identities is a $\lambda$-identity. It can be proved that a variety defined by a set of identities is a $\lambda$-variety if and only if all identities of this set are $B_{0}$-identities and one of them is a $\lambda$-identity.

The same property is valid for the $\rho^{-}, \lambda^{*}-, \rho^{*}-, \bar{\lambda}-$, and $\bar{\rho}$-varieties, which are defined similarly.

7. A nonpermutation identity of the form $x_{1} x_{2} \ldots x_{n}=u$ is called a diverse identity. A variety $V$ of semigroups in which a diverse identity is valid is said to be diverse. Such varieties are also called varieties of finite degree (see [1]).

8. We fix the notation for some finite semigroups that are used repeatedly in what follows. As usual, braces and angle braces are used for semigroups defined by multiplication tables and defining relations, respectively. In the sequel, $p$ is a prime, and, for a semigroup 
defined by a multiplication table (curve braces), the skipped products are zero.

$$
\begin{aligned}
& L_{2}=\left\{e, f \mid e^{2}=e, f^{2}=f, \text { ef }=e, f e=f\right\} ; \\
& R_{2}=\left\{e, f \mid e^{2}=e, f^{2}=f, \text { ef }=f, f e=e\right\} ; \\
& N_{1}=\left\{x, 0 \mid x^{2}=0\right\} ; \\
& Y_{2}=\left\{e, 0 \mid e^{2}=e\right\} ; \\
& A_{0}=\left\{e, f, z, 0 \mid e^{2}=e, f^{2}=f, e f=e z=z f=z\right\} ; \\
& B_{0}=\left\{e, f, z, 0 \mid e^{2}=e, f^{2}=f, e z=z f=z\right\} ; \\
& M_{p}=\left\langle x, y \mid x^{2}=x, y^{2}=y, x=(x y)^{p+1} x, y=(y x)^{p+1} y\right\rangle ; \\
& A_{2}=\left\langle a, b \mid a^{2}=a b a=a, b^{2}=0, b a b=b\right\rangle ; \\
& B_{2}=\left\langle a, b \mid a^{2}=b^{2}=0, a b a=a, b a b=b\right\rangle ; \\
& B_{l}=\langle e, f, g, a, 0| g^{2}=g, e^{2}=e, f^{2}=f, e f=e, f e=f, g a=a, \\
&\left.\quad a^{2}=a g=a f=e a=f a=g e=e g=f g=g f=0\right\rangle ; \\
&\left.A_{l}^{*}=\langle e, f, g, 0| g^{2}=g, e^{2}=e, f^{2}=f, \text { ef }=e, f e=f, e g=f g=0, g f=0\right\rangle ;
\end{aligned}
$$

$A_{r}^{*}, B_{r}$ are the right analogs of the semigroups $A_{l}^{*}, B_{l}$.

9. We recall that an element $b$ of a semigroup $S$ is said to be cyclic if $b$ can be represented in the form $b=a x a$ for $a \in S$ and an element $x \in S$ (or the empty symbol). A semigroup $S$ is cyclically regular if every regular element in $S$ is cyclic. A semigroup $S$ is orthodox if the set of idempotents in $S$ is a subsemigroup of $S$. A semigroup $S$ is called a Rees-Sushkevich semigroup if $S \in R S_{n}$ for some $n$.

10. We introduce some series of finite semigroups depending on a positive integer parameter $k$ :

$$
\begin{array}{r}
S L_{k}=\left\langle e, f, z_{1}, z_{2}, \ldots, z_{k}, 0\right| e^{2}=e, f^{2}=f, \text { ef }=e, f e=f, z_{i} z_{j}=0(j-i \neq 1), \\
\left.e z_{i}=f z_{i}=0(i=1,2, \ldots, k), z_{j} z_{j+1} e=z_{j} e=z_{j} f(j \neq k), z_{k} f=0\right\rangle ;
\end{array}
$$

$S L_{k}^{*}$ is the subsemigroup of $S L_{k}$ generated by the elements $e, z_{1}, z_{2}, \ldots, z_{k}$;

$S R_{k}$ and $S R_{k}^{*}$ are the right analogs of the semigroups $S L_{k}$ and $S L_{k}^{*}$;

$N_{k}$ is the free semigroup with $k$ generators in the variety

$$
\left[x^{2}=0, x y x=0\right]
$$

$C N_{k}$ is the free semigroup with $k$ generators in the variety

$$
\left[x^{2}=0, x y x=0, x y=y x\right] .
$$

11. Let $V$ be a variety of semigroups. We denote by $n_{C N}(V)$ the largest $k$ such that the semigroup $C N_{k}$ belongs to $V$. If all semigroups $C N_{k}$ belong to $V$, we put $n_{C N}(V)=\infty$, and if there are no such semigroups in $V$, then we put $n_{C N}(V)=0$. The numbers $n_{N}(V)$, $n_{S L}(V), n_{S L}^{*}(V), n_{S R}(V)$, and $n_{S R}^{*}(V)$ are defined similarly.

12. Let $V$ be a variety of semigroups. We denote by $n_{D}(V)$ the sum $n_{C N}(V)+1$ (if $n_{C N}(V)=\infty$, then we assume that $\left.n_{D}(V)=\infty\right)$. The number $n_{D}(V)$ is called the diversity degree of $V$. If $n_{D}(V) \neq \infty$, then $n_{D}(V)$ is the smallest positive integer $k$ for which an identity of the form $x_{1} x_{2} \ldots x_{k}=w$ (the so-called diverse identity) is valid in $V$. Without loss of generality, we may assume that $|w|>k$. The number $n_{D}(V)$ is also called the degree of $V$, because $n_{D}(V)$ is equal to the maximal nilpotency degree of the nilpotent semigroups in $V$. 
13. Let $V$ be a variety of semigroups, and let $S$ be a semigroup. We put $\epsilon_{S}(V)=1$ if $S \in V$ and $\epsilon_{S}(V)=0$ if $S \notin V$.

14 (The rank). Let $V$ be a variety of semigroups. The Rees-Sushkevich rank $\operatorname{Rank}(V)$ of $V$ is the following ordered set of 12 nonnegative integers (or symbols $\infty$ ):

$$
\left(\vec{V}, n_{C N}(V), n_{N}(V), n_{S L}(V), n_{S R}(V), n_{S L}^{*}(V), n_{S R}^{*}(V)\right)
$$

where $\vec{V}=\left(\epsilon_{B_{0}}(V), \epsilon_{A_{0}}(V), \epsilon_{B_{l}}(V), \epsilon_{B_{r}}(V), \epsilon_{A_{l}^{*}}(V), \epsilon_{A_{r}^{*}}(V)\right)$. We say that $\operatorname{Rank}\left(V_{1}\right) \leq$ $\operatorname{Rank}\left(V_{2}\right)$ if the inequality holds true componentwise.

15 (Rees-Sushkevich radical and semisimple varieties). Let $V$ be a variety of semigroups. The radical $\mathfrak{R}(V)$ of $V$ is the variety defined by the identities $x^{2}=0$, $x y x=0, x_{1} \ldots x_{n_{D}(V)}=0$ (if $n_{D}(V) \neq \infty$ ), and all permutation identities in $V$ of length less than the degree $n_{D}(V)$ of $V$. If there are no such permutation identities in $V$, we say that the radical of $V$ is zero, write $\mathfrak{R}(V)=0$, and assume that the variety $\mathfrak{R}(V)$ is trivial. A variety with zero radical is said to be semisimple (in other words, a variety $V$ is semisimple if the length of each permutation identity in $V$ is at least the degree of $V$ ). A variety $V$ is said to be radical if $V$ coincides with its radical. It is easy to prove that the radical of a variety $V$ of semigroups is a radical subvariety of $V$, i.e., $\mathfrak{R}(\mathfrak{R}(V))=\mathfrak{R}(V)$.

16. Let $V$ be a variety of semigroups. We denote by $\mathfrak{N}_{V}$ the set of finite semigroups in $\mathfrak{R}(V)$, by $\mathfrak{A}$ be the set formed by the semigroups $B_{0}, A_{0}, B_{l}, B_{r}, A_{l}^{*}, A_{r}^{*}, N_{k}, C N_{k}$, $S L_{k}, S R_{k}, S L_{k}^{*}$, and $S R_{k}^{*}(k=1,2, \ldots)$, and by $\mathfrak{A}_{V}$ the intersection of $\mathfrak{A}$ and $V$.

17 (Left (right) decompositions of an identity). Every word $u$ can uniquely be represented in the form $u \equiv w_{u} u^{\prime}$, where either $w_{u}$ is the empty word, or each letter in $w_{u}$ occurs in $u$ only once, and either $u^{\prime}$ is the empty word, or the first letter of $u^{\prime}$ occurs in $u^{\prime}$ more than once. This representation of $u$ will be called the left decomposition of $u$. If $u \equiv w_{u} u^{\prime}$ and $v \equiv w_{v} v^{\prime}$ are the left decompositions of $u$ and $v$, respectively, then the identity $w_{u} u^{\prime}=w_{v} v^{\prime}$ is called the left decomposition of the identity $u=v$. The right decompositions are defined by dualization.

Example. The left decomposition of the identity $x y z^{2} t y t^{3}=t z x y x$ looks like this: $x\left(y z^{2} t y t^{3}\right)=t z(x y x)$.

18. We introduce the following notation for identities used repeatedly in the sequel:

$$
\begin{aligned}
e q_{1} & : x^{n} y^{m}=u y x v ; \\
e q_{2} & : x^{n} z y^{m}=w z w^{*}, c(w) \cap c\left(w^{*}\right) \neq \varnothing ; \\
e q_{1, n} & :\left(x^{2 n} y^{2 n}\right)^{n+1}=x^{2 n} y^{2 n} ; \\
e q_{2, n} & : x^{2 n} y z^{2 n}=x^{2 n} y^{n+1} z^{2 n} ; \\
e q B_{l, n} & : z^{2 n} t x^{2 n} y^{2 n} x^{2 n} y^{2 n}=z^{2 n} t y^{2 n} x^{2 n} y^{2 n} ; \\
e q B_{r, n} & : x^{2 n} y^{2 n} x^{2 n} y^{2 n} t z^{2 n}=x^{2 n} y^{2 n} x^{2 n} t z^{2 n} ; \\
e q A_{l, n}^{*} & : z^{2 n} x^{2 n} y^{2 n} x^{2 n} y^{2 n}=z^{2 n} y^{2 n} x^{2 n} y^{2 n} ; \\
e q A_{r, n}^{*} & : x^{2 n} y^{2 n} x^{2 n} y^{2 n} z^{2 n}=x^{2 n} y^{2 n} x^{2 n} z^{2 n}
\end{aligned}
$$


We also introduce the following notation for sets of identities:

$$
\begin{aligned}
E q N^{(S L, n)} & =\left\{x_{1} x_{2} \ldots x_{m} x^{2 n} y^{2 n} x^{2 n} y^{2 n}=x_{1} x_{2} \ldots x_{m} y^{2 n} x^{2 n} y^{2 n} \mid m \in \mathbb{N}\right\} \\
E q N^{(S R, n)} & =\left\{x^{2 n} y^{2 n} x^{2 n} y^{2 n} x_{1} x_{2} \ldots x_{m}=x^{2 n} y^{2 n} x^{2 n} x_{1} x_{2} \ldots x_{m}, \mid m \in \mathbb{N}\right\} \\
E q N^{(l, n)} & =\left\{x_{1} x_{2} \ldots x_{m} y^{2}=x_{1} x_{2} \ldots x_{m}^{n+1} y^{2} \mid m \in \mathbb{N}\right\} \\
E q N^{(r, n)} & =\left\{y^{2} x_{1} x_{2} \ldots x_{m}=y^{2} x_{1}^{n+1} x_{2} \ldots x_{m} \mid m \in \mathbb{N}\right\} ; \\
E q D^{(n)} & \text { is the set of all identities of the form } \\
& x_{1} x_{2} \ldots x_{k_{1}} z_{1} z_{2} \ldots z_{k_{2}} y_{1} y_{2} \ldots y_{k_{3}}=x_{1} x_{2} \ldots x_{k_{1}}\left(z_{1} z_{2} \ldots z_{k_{2}}\right)^{n+1} y_{1} y_{2} \ldots y_{k_{3}},
\end{aligned}
$$

where $k_{1}$ and $k_{3}$ are nonnegative integers and $k_{2}$ is a positive integer.

We denote by $E q R S_{n}$ the set consisting of the identities $e q_{1, n}, e q_{2, n}, e q B_{l, n}, e q B_{r, n}$, $e q A_{l, n}^{*}, e q A_{r, n}^{*}$, and all identities belonging to the sets $E q N^{(S L, n)}, E q N^{(S R, n)}, E q N^{(l, n)}$, $E q N^{(r, n)}$, and $E q D^{(n)}$. We denote by $E q R S[n]$ the union $E q R S_{n} \cup E q r e g \cup E q N$, where Eqreg is the set of all identities of the form $u=v$ with regular $u$ and $v$. Let $E q N$ be the set of all permutation identities. Also, we denote by $E q R S$ the set of all identities listed above for all $n$.

\section{$\S 3$. The MAIn RESUlts}

Here, we formulate the main results of the present paper (Theorems 1-5) and their applications (Corollaries 1-16) devoted to the description of the Rees-Sushkevich varieties satisfying finiteness conditions. The proofs of these results are given below in a separate section.

Theorem 1. Every Rees-Sushkevich variety $V$ is generated by the set $C S^{0} V$ of completely 0-simple semigroups in $V$ and the set $\mathfrak{A}_{V} \cup \mathfrak{N}_{V}$ of finite semigroups (the sets $\mathfrak{A}_{V}$ and $\mathfrak{N}_{V}$ are defined in Subsection 2.16).

Theorem 2. Let $V_{1}$ and $V_{2}$ be semisimple Rees-Sushkevich varieties. If $V_{1}^{\prime}=V_{2}^{\prime}$ and $\operatorname{Rank}\left(V_{1}\right)=\operatorname{Rank}\left(V_{2}\right)$, then $V_{1}=V_{2}$.

Theorem 3. Every Rees-Sushkevich variety $V$ is the sum of the radical of $V$ and a semisimple variety.

Theorem 4. Every Rees-Sushkevich variety of period $n$ can be defined by the following set of identities:

$\left(\mathrm{I}_{n}\right) x^{2}=x^{n+2}$

$\left(\mathrm{II}_{n}\right) x y x=(x y)^{n+1} x$

$\left(\mathrm{III}_{n}\right)(x h z)^{n} \cdot x y z=x y z \cdot(x h z)^{n}$

and a set of identities belonging to $\operatorname{EqRS}[n]$.

Theorem 5. Let $V_{1}$ and $V_{2}$ be arbitrary Rees-Sushkevich varieties. If $V_{1}^{\prime}=V_{2}^{\prime}$ and $\operatorname{Rank}\left(V_{1}\right)=\operatorname{Rank}\left(V_{2}\right)$, then in $V_{1}$ and $V_{2}$ there exist bases of identities that differ only by collections of permutation identities.

Corollary 1 (Exactness criterion). A Rees-Sushkevich variety $V$ is exact if and only if at least one of the following conditions is fulfilled:

(C1) $A_{2}$ is contained in $V$;

(C2) $N_{1}$ is not contained in $V$;

(C3) $B_{2}$ and $M_{p}$ are contained in $V$ for some prime $p$, but $A_{0}$ is not;

(C4) $B_{2}$ is contained in $V$, but $A_{0}, S L_{1}$, and $S R_{1}$ are not. 
Remark 1. A similar criterion was obtained by Reilly independently in [16]. Reilly's criterion differs from the above only in condition $(C 4)$, whose role in [16] was played by the following condition: an identity of the form $t x^{2 n} y^{2 n} s=t y^{2 n} x^{2 n} s$ is fulfilled in $V$. Since the inference problem for semigroup identities is algorithmically undecidable (see [4]), we see that, in contrast to the criterion given above, Reilly's criterion does not allow one to decide by a finite basis of identities in a variety $V$ whether or not $V$ is exact.

Corollary 2. Let $V$ be a periodic variety of semigroups. If $V$ is finitely based, then so is $V^{\prime}$.

Corollary 3 (Description of the exact combinatorial varieties). Let $V$ be an exact combinatorial variety (i.e., $V \subset R S_{1}$ and $V$ is generated by completely 0-simple semigroups). Then $V$ is one of the following varieties:

1) $V=[x=y]$;

2) $V=\operatorname{Var}\left(Y_{2}\right)=\left[x^{2}=x, x y=y x\right]$;

3) $V=\operatorname{Var}\left(L_{2}\right)=[x y=x]$;

4) $V=\operatorname{Var}\left(R_{2}\right)=[x y=y]$

5) $V=\operatorname{Var}\left(L_{2} \times R_{2}\right)=[x y x=x]$;

6) $V=\operatorname{Var}\left(L_{2} \times Y_{2}\right)=\left[x^{2}=x, a x y=a y x\right]$;

7) $V=\operatorname{Var}\left(R_{2} \times Y_{2}\right)=\left[x^{2}=x, x y a=y x a\right]$;

8) $V=\operatorname{Var}\left(L_{2} \times Y_{2} \times R_{2}\right)=\left[x^{2}=x\right.$, axya $=$ ayxa $]$;

9) $V=\operatorname{Var}\left(B_{2}\right)=\left[x^{2}=x^{3}, x y x=x y x y x, x^{2} y^{2}=y^{2} x^{2}\right]$;

10) $V=\operatorname{Var}\left(L_{2} \times B_{2}\right)=\left[x^{2}=x^{3}, x y x=x y x y x, a x^{2} y^{2}=a y^{2} x^{2}\right]$;

11) $V=\operatorname{Var}\left(B_{2} \times R_{2}\right)=\left[x^{2}=x^{3}, x y x=x y x y x, x^{2} y^{2} a=y^{2} x^{2} a\right]$;

12) $V=\operatorname{Var}\left(L_{2} \times B_{2} \times R_{2}\right)=\left[x^{2}=x^{3}, x y x=x y x y x, a x^{2} y^{2} b=a y^{2} x^{2} b\right]$;

13) $V=\operatorname{Var}\left(A_{2}\right)=\left[x^{2}=x^{3}\right.$, xyx $=$ xyxyx, xyxzx $\left.=x z x y x\right]=R S_{1}$.

We denote by $S^{\prime}$ the 0 -direct product of the completely 0-simple principal factors of the semigroup $S$ (see [7]) and call the semigroup $S^{\prime}$ the derivative of $S$.

Corollary 4 (A criterion for a variety to be finitely based). A Rees-Sushkevich variety $V$ is finitely based (hereditarily finitely based) if and only if the derivative $V^{\prime}$ has the same property.

Corollary 5. A Rees-Sushkevich semigroup $S$ is finitely based (hereditarily finitely based) if and only if the derivative $S^{\prime}$ is finitely based (hereditarily finitely based).

Corollary 6. A finite orthodox Rees-Sushkevich semigroup is hereditarily finitely based.

Corollary 7 (E. Lee [10, 11]). The variety $R S_{1}$ is hereditarily finitely based.

Corollary 8. Let $V$ be a Rees-Sushkevich variety, and let $\left[V^{\prime}, V\right]$ be an interval in the lattice of subvarieties of $V$. Then $\left[V^{\prime}, V\right]$ satisfies the minimality condition and contains no infinite antichains.

Corollary 9. A Rees-Sushkevich variety $V$ satisfies the minimality condition or contains no infinite antichains if and only if the derivative $V^{\prime}$ has the same property. In particular, the variety $R S_{1}$ satisfies the minimality condition and contains no infinite antichains. Therefore, each subvariety in $R S_{1}$ has finitely many covers.

Corollary 10. A Rees-Sushkevich variety $V$ has finite width if and only if the derivative variety $V^{\prime}$ has the same property and either $V$ is a diverse variety, or a permutation identity is fulfilled in $V$. 
Corollary 11. Let $V$ be a Rees-Sushkevich variety. The following conditions are equivalent:

1) the interval $\left[V^{\prime}, V\right]$ is finite;

2) the interval $\left[V^{\prime}, V\right]$ satisfies the maximality condition;

3) $V$ satisfies one of the following conditions: $V$ is diverse; $V$ contains $A_{2} ; V$ contains $B_{2}$ and contains $M_{p}$ (for a prime $\left.p\right) ; V$ contains $B_{2}$ and is a $\lambda^{*}$ - and a $\rho^{*}$-variety.

Corollary 12. Let $V$ be a Rees-Sushkevich variety. The following statements are equivalent.

1) $V$ is generated by a finite semigroup and a completely 0 -simple semigroup.

2) The following conditions are satisfied:

$(C F 1)$ if $V$ is a $\lambda$-variety, then $V$ is a $\lambda^{*}$-variety;

$(C F 2)$ if $V$ is a $\rho$-variety, then $V$ is a $\rho^{*}$-variety;

$(C F 3)$ if $B_{0} \notin V$, then $V$ is a diverse variety.

Remark 2. Corollaries 11 and 12 and the definitions show that the finiteness conditions stated in these corollaries can easily be verified for the Rees-Sushkevich varieties defined by a finite semigroup or by a finite basis of identities.

Corollary 13 (A criterion for finite generation). Let $V$ be a Rees-Sushkevich variety. The following statements are equivalent.

1) $V$ is generated by a finite semigroup.

2) The derivative variety $V^{\prime}$ is finitely generated and the following conditions are fulfilled:

$(C F 1)$ if $V$ is a $\lambda$-variety, then $V$ is a $\lambda^{*}$-variety;

$(C F 2)$ if $V$ is a $\rho$-variety, then $V$ is a $\rho^{*}$-variety;

$(C F 3)$ if $B_{0} \notin V$, then $V$ is diverse.

Corollary 14. A Rees-Sushkevich variety $V$ satisfies the maximality condition if and only if the derivative variety $V^{\prime}$ has the same property and $V$ is diverse.

Remark 3. For the combinatorial Rees-Sushkevich varieties (i.e., those belonging to $R S_{1}$ ), we can drop the requirement on the derivative in Corollaries 10, 13, and 14,

Corollary 15. In the lattice of subvarieties of $R S_{n}$, the union and the covers of the varieties satisfying the maximality condition satisfy the maximality condition.

This corollary answers Question 10.2 in [1] for the Rees-Sushkevich varieties.

Corollary 16 (A criterion for being small (Cross)). A Rees-Sushkevich variety $V$ is small (Cross) if and only if the derivative $V^{\prime}$ of $V$ has the same property and $V$ is a diverse variety. In particular, a variety $V$ included in $R S_{2}$ is small (Cross) if and only if $V$ is diverse.

Remark 4. This generalizes the main result of the paper [12] by E. Lee, where this fact was proved for varieties included in $R S_{1}$. In the proof in the present paper, we do not lean upon Lee's results.

\section{§4. Algorithmic Questions (a List)}

Here, we list some applications of the results obtained to the solution of a series of algorithmic problems for Rees-Sushkevich varieties. The proofs of these results are given below in a separate section.

Theorem 6. For a variety $V$ of semigroups defined by a finite number of identities, there is an algorithm linear in the sum of the lengths of the basis identities and allowing one to calculate the rank of $V$. 
Theorem 7. Let $V$ be a Rees-Sushkevich variety defined by a finite semigroup or by a finite set of identities. The variety $V$ has a decidable (polynomially decidable) equational theory if and only if the derivative $V^{\prime}$ of $V$ has the same property.

Corollary 17. Every Rees-Sushkevich variety included in $R S_{1}$ and given by a finite semigroup or by a finite set of identities has a polynomially decidable equational theory.

Theorem 8. Let $V$ be an arbitrary periodic variety of semigroups defined by a finite semigroup or by a finite set of identities. There is an algorithm of polynomial complexity allowing one to decide whether or not $V$ is generated by completely 0-simple semigroups.

\section{§5. Auxiliary Results}

In this section, we give some auxiliary statements used in the proofs of the main results. Some of these statements are of independent interest. The following three claims follow from the definitions.

Lemma 1. If $V_{1} \subset V_{2}$, then $\operatorname{Rank}\left(V_{1}\right) \leq \operatorname{Rank}\left(V_{2}\right)$.

Lemma 2. $N_{k} \notin V$ if and only if an identity of the form $x_{1} x_{2} x_{3} \ldots x_{k}=w$, where $w \not \equiv x_{1} x_{2} x_{3} \ldots x_{k}$ and $|w| \geq k$, is fulfilled in $V$.

Lemma 3. $C N_{k} \notin V$ if and only if an identity of the form $x_{1} x_{2} x_{3} \ldots x_{k}=w$, where $|w|>k$, is fulfilled in $V$.

Lemma 4 (On regular elements). Let $V$ be included in $R S_{n}$ and $S$ in $V$. For any two nonequal regular elements $u$ and $v$ of $S$, there exists a completely 0 -simple principal factor $K$ of the semigroup $S$ and an epimorphism $f: S \rightarrow K$ such that $f(u) \neq f(v)$.

Proof. This statement was proved in the author's paper [9].

Lemma 5. The semigroups $A_{0}, B_{0}, B_{l}, B_{r}, A_{l}^{*}, A_{r}^{*}, S L_{k}$, and $S R_{k}$ admit exact Rees matrix representations over the field of two elements; therefore, they belong to $R S_{1}$. The semigroups $N_{k}$ and $C N_{k}$ belong to $R S_{1}$ by definition.

Proof. We prove that these semigroups have exact Rees matrix representations over the field of two elements. For $A_{0}$ and $B_{0}$, this is well known because the semigroups in question are subsemigroups of the Rees matrix semigroups $A_{2}$ and $B_{2}$, respectively. We consider each of the remaining semigroups separately.

1. We define a map from $\{g, a, e, f\} \subset B_{l}$ to the Rees matrix combinatorial semigroup $S$ with the sandwich matrix

$$
\left(\begin{array}{lll}
1 & 0 & 0 \\
0 & 1 & 1 \\
0 & 1 & 0
\end{array}\right)
$$

(see [7]) by the rule $g \rightarrow(1,1), a \rightarrow(1,3), e \rightarrow(2,2), f \rightarrow(3,2)$. It can easily be verified that the images satisfy the same relations as those valid in $B_{l}$. Consequently, the map in question can be extended to a homomorphism $\gamma: B_{l} \rightarrow S$. It is easy to check that the homomorphism $\gamma$ is injective. Thus, the semigroup $B_{l}$ belongs to $R S_{1}$, being a subsemigroup of the Rees combinatorial matrix semigroup $S$. The proof for the semigroup $B_{r}$ is similar.

2. We define a map from $\{g, e, f\} \subset A_{l}^{*}$ to the Rees combinatorial matrix semigroup $S$ with the sandwich matrix

$$
\left(\begin{array}{lll}
1 & 1 & 0 \\
0 & 1 & 1 \\
0 & 1 & 0
\end{array}\right)
$$


by the rule $g \rightarrow(1,1), e \rightarrow(2,2), f \rightarrow(3,2)$. It can easily be verified that the images satisfy the relations valid in the semigroup $A_{l}^{*}$. Consequently, the map under consideration can be extended to a homomorphism $\gamma: A_{l}^{*} \rightarrow S$. It is easy to check that the homomorphism $\gamma$ is injective. Thus, the semigroup $A_{l}^{*}$ belongs to $R S_{1}$, being a subsemigroup of the matrix combinatorial semigroup $S$. The statement for $A_{r}^{*}$ is proved similarly.

3. We define a map from $\left\{e, f, z_{1}, z_{2}, \ldots, z_{k}\right\} \subset S L_{k}$ to the Rees combinatorial matrix semigroup $S$ with the sandwich matrix

$$
\left(\begin{array}{ccccccc}
1 & 0 & \ldots & 0 & 0 & 1 & 1 \\
0 & 1 & \ldots & 0 & 0 & 1 & 1 \\
\ldots & \ldots & \ldots & \ldots & \ldots & \ldots & \ldots \\
\ldots & \ldots & \ldots & 1 & 0 & 1 & 1 \\
0 & \ldots & \ldots & 0 & 1 & 1 & 0 \\
0 & \ldots & \ldots & 0 & 0 & 1 & 1 \\
0 & \ldots & \ldots & 0 & 0 & 1 & 0
\end{array}\right)
$$

of size $(k+3) \times(k+3)$ by the rule

$$
z_{i} \rightarrow(i, i+1), \quad e \rightarrow(k+2, k+2), \quad f \rightarrow(k+3, k+2) .
$$

It is easy to verify that the images satisfy the relations valid in the semigroup $S L_{k}$. Consequently, the map in question can be extended to a homomorphism $\gamma: S L_{k} \rightarrow S$. It is easily seen that the homomorphism $\gamma$ is injective. Thus, the semigroup $S L_{k}$ belongs to $R S_{1}$, being a subsemigroup of the Rees combinatorial matrix semigroup $S$. For the semigroup $S R_{k}$, the statement is proved similarly. The semigroups $S L_{k}^{*}$ and $S R_{k}^{*}$ are subsemigroups of $S L_{k}$ and $S R_{k}$, respectively. Therefore, $S L_{k}^{*}$ and $S R_{k}^{*}$ also belong to the variety $R S_{1}$.

Proposition 6. Let $V$ be a variety of semigroups. The following properties of $V$ are equivalent.

(1) For each cyclically regular semigroup $S$ in $V$, the set $r S$ of regular elements of $V$ is a biideal, i.e., $r S \cdot S \cdot r S \subset r S$.

(2) $B_{0} \notin V$.

(3) An identity $u=v$ with noncongruent words $u$ and $v$ is fulfilled in $V$.

(4) An identity eq $: x^{n} z y^{m}=w z w^{*}$, where $c(w) \cap c\left(w^{*}\right) \neq \varnothing$, is fulfilled in $V$.

Proof. (1) $\Rightarrow(2)$. Assume that (1) is valid. This means that the set of regular elements of an arbitrary cyclically regular semigroup of $V$ is a biideal. In the semigroup $B_{0}$, we have $z=e z f$, but $z$ is not a regular element because $z x z=0$ for every $x \in B_{0}$. Consequently, property (1) is not fulfilled in $B_{0}$, whence $B_{0} \notin V$.

$(2) \Rightarrow(3)$. This is well known (see, e.g., [12]).

$(3) \Rightarrow(4)$. We assume that (3) is valid in $V$. This means that an identity $u=v$ such that $u$ and $v$ are not congruent is fulfilled, but the identity $e q_{2}: x^{n} z y^{m}=w z w^{*}$ is not.

Let $c(u) \neq c(v)$. Then $u=v$ implies an identity of the form $x^{n}=P y Q$, which implies $e q_{2}: x^{n} z y^{m}=w z w^{*}$.

Now, let $c(u)=c(v)$. We recall that a letter $t$ of a word $u$ is said to be blocking if $u$ has the form $u \equiv u_{1} t u_{2}$, where no two of the words $t, u_{1}$, and $u_{2}$ have a common letter. We prove that the sets of blocking letters of $u$ and $v$ coincide.

Suppose the contrary. Then there exists a letter $t$ blocking in $u$ but not in $v$ (or vice versa). By definition, we conclude that the words $u$ and $v$ have the form

$$
u \equiv u_{1} t u_{2}, \quad v \equiv a z w z b .
$$

Here, $a, b$, and $w$ are words or empty symbols, $z$ is a letter, the letter $t$ occurs in $z w z$ but does not occur in the words $u_{1}$ and $u_{2}$, and the words $u_{1}$ and $u_{2}$ have no common 
letters. Let $z \equiv t$. Then, substituting the element $x$ for each letter of the word $u_{1}$, the element $y$ for each letter of the word $u_{2}$, and the element $x t y$ for $t$ in the identity $u=v$, we obtain an identity of the form $e q_{2}: x^{n} z y^{m}=w z w^{*}$, a contradiction.

If $z \not \equiv t$, then $t$ occurs in the word $w$. Therefore, substituting the element $x$ for each letter of $u_{1}$ and the element $y$ for each letter of $u_{2}$ in the identity $u=v$, we obtain an identity of the form $e q_{2}: x^{n} z y^{m}=w z w^{*}$, a contradiction.

Thus, we have proved that the sets of blocking letters of the words $u$ and $v$ coincide. Consequently, if one of the words $u$ or $v$ is singular, then the other is also singular. However, in this case, $u=v$ is a permutation identity. It follows that an identity of the form $x_{1} x_{2} \ldots x_{k} y_{1} y_{2} z_{1} z_{2} \ldots z_{k}=x_{1} x_{2} \ldots x_{k} y_{2} y_{1} z_{1} z_{2} \ldots z_{k}$ is fulfilled for some positive integer $k$ in $V$ (see [14]). Substituting the letter $x$ for $x_{i}$, the letter $t$ for $y_{1}$, and the letter $y$ for $y_{2}$ and $z_{j}$, we obtain $e q_{2}: x^{n} z y^{m}=w z w^{*}$, a contradiction.

Thus, we may assume that the words $u$ and $v$ are nonsingular. Let $t$ be a blocking letter of the words $u$ and $v$, and let $z$ be a nonblocking letter. We assume that, in one word (say, in $u$ ), the letter $z$ is to the left of $t$, and, in the other word, the letter $t$ is to the left of $z$. We substitute the element $x^{2 n}$ for the letter $z$ and the element $y^{2 n}$ for the letters distinct from $z$ and $t$ in the identity $u=v$. Multiplying the new identity from the left by $x^{2 n}$ and from the right by $y^{2 n}$, we obtain an identity of the form $e q_{2}: x^{n} z y^{m}=w z w^{*}$, a contradiction.

Let $t_{1}$ and $t_{2}$ be two different blocking letters of the words $u$ and $v$. We assume that, in one word (say, in $u$ ), the letter $t_{1}$ is to the left of $t_{2}$, and that the letter $t_{2}$ is to the left of $t_{1}$ in the other word. In the identity $u=v$, we substitute the element $x^{2 n}$ for the letters $z$ that are to the left of $t_{2}$ in the word $u$, and substitute the element $t$ for the letter $t_{2}$ and the element $y^{2 n}$ for the letters $z$ that are to the right of $t_{2}$ in $u$. Multiplying the new identity from the left by $x^{2 n}$ and from the right by $y^{2 n}$, we obtain an identity of the form $e q_{2}$, a contradiction.

Let

$$
\begin{aligned}
& u=w_{1}^{(u)} u_{1}^{\#} w_{2}^{(u)} u_{2}^{\#} \ldots w_{n(u)}^{(u)} u_{n(u)}^{\#} w_{n(u)+1}^{(u)}, \\
& v=w_{1}^{(v)} v_{1}^{\#} w_{2}^{(v)} v_{2}^{\#} \ldots w_{n(v)}^{(v)} v_{n(v)}^{\#} w_{n(v)+1}^{(v)}
\end{aligned}
$$

be canonical decompositions of the 2 nd kind for the words $u$ and $v$, respectively. As was proved above, the numbers, as well as the orders, of the blocking letters in the words $u$ and $v$ coincide. The orders of precedence of the blocking and nonblocking letters also coincide. If $c(u)=c(v)$, then this is possible only if $n(u)=n(v), w_{i}(u)=w_{i}(v)$, and $c\left(u_{i}^{\#}\right)=c\left(v_{i}^{\#}\right)$ for all values of $i$. Consequently, the words $u$ and $v$ are congruent. This contradiction proves that $(3) \Rightarrow(4)$.

$(4) \Rightarrow(1)$. Let an identity of the form $e q_{2}: x^{n} z y^{m}=w z w^{*}$ be fulfilled in $V$. In this identity, we replace the letter $z$ by $x y$, and the remaining letters distinct from $x, y$, and $z$ by $x$. This yields an identity of the form $e q_{1}: x^{n} y^{n}=u y x v$. By Proposition 7 the variety $V$ is regularly closed. Let $S$ be a cyclically regular semigroup in $V$. We assume that $z \in c\left(w w^{*}\right)$. This means that the identity $e q_{2}$ has the form $x^{n} z y^{m}=P z Q z R$. This implies an identity of the form

$$
x^{n+1} z y^{m+1}=P^{\#} x z y Q^{\#} x z y R^{\#} .
$$

Without loss of generality, we may assume that $P^{\#}, Q^{\#}$, and $R^{\#}$ are words in the variables $x, y$, and $z$. The word $P^{\#} x z y Q^{\#} x z y R^{\#}$ is regular by definition. We conclude that, for all idempotents $e$ and $f$ in $S$ and all $t$ in $S$, the element et $f$ is equal to the value of some regular word.

As was established by the author in [21, in a cyclically regular semigroup, the values of regular words are regular elements. Therefore, et $f$ is a regular element of $S$. 
Let $a$ and $b$ be arbitrary regular elements of $S$, and let $a=a a^{\prime} a$ and $b=b b^{\prime} b$. Then $a t b=a\left(\left(a^{\prime} a\right) t\left(b b^{\prime}\right)\right) b$ is a product of three regular elements. Since $S$ is regularly closed, this product is a regular element, i.e., atb is a regular element. Therefore, $(4) \Rightarrow(1)$. Proposition 6 is proved completely.

Proposition 7. Let $V$ be a variety of semigroups. The following conditions are equivalent.

(1) All semigroups in $V$ are regularly closed.

(2) The variety $V$ does not contain the semigroup $A_{0}$.

(3) Each basis of $V$ contains an identity $u=v$ such that the words $u$ and $v$ are not similar.

(4) An identity of the form $e q_{1}: x^{n} y^{n}=u y x v$ is fulfilled in $V$.

Proof. Largely, the proof is similar to that of Proposition 6. It suffices to observe that the semigroup $A_{0}$ is not regularly closed and that all identities in which the left- and right-hand sides are similar and only such identities are fulfilled in $A_{0}$ (see [21]).

Proposition 8. Let $V \subset R S_{n}$ be a $B_{0}$-Sushkevich variety. The following properties are equivalent.

(1) $V$ is a $\lambda$-variety;

(2) an identity of the form

$$
z^{2 n} t x^{2 n} y^{2 n} x^{2 n} y^{2 n}=z^{2 n} t y^{2 n} x^{2 n} y^{2 n}
$$

is fulfilled in $V$.

Proof. (1) $\Rightarrow(2)$. Let $V$ be a $\lambda$-variety. This means that a $B_{0}$-identity

$$
w_{1} u_{1} w_{2} u_{2} \ldots w_{m} u_{m} w_{m+1}=w_{1} v_{1} w_{2} v_{2} \ldots w_{m} v_{m} w_{m+1},
$$

such that $h\left(u_{i}\right) \neq h\left(v_{i}\right)$ for some $i$ is fulfilled in $V$. It can easily be shown (see [5]) that such an identity cannot be valid in the semigroup $M_{p}$, which is a completely simple semigroup over the cyclic group $Z(p)$ of prime order with the sandwich-matrix $\left(\begin{array}{ll}1 & 1 \\ 1 & a\end{array}\right)$, where $a$ is a generator of $Z(p)$. Therefore, we can conclude that all completely simple semigroups in $V$ are orthodox (we recall that a semigroup $S$ is said to be orthodox if the product of arbitrary idempotents in $S$ is an idempotent in $S$ ). This means that, for every word $f$ in the variables $x^{2 n}, y^{2 n}$, the following identity is fulfilled in $V$ :

$$
x^{2 n} y^{2 n} x^{2 n} f\left(x^{2 n}, y^{2 n}\right) y^{2 n}=x^{2 n} y^{2 n} x^{2 n} y^{2 n} .
$$

Indeed, assuming the contrary, we can find a semigroup $S \in V$ such that the above identity fails for some $x$ and $y$ in $S$. Since the words in $(* *)$ are regular and the values of regular words are regular elements in the Rees-Sushkevich semigroups (see [9]), we can apply Lemma 4 about regular elements. This shows that identity $(* *)$ is not fulfilled in some completely 0 -simple semigroup in $V$. We note that identity $(*)$ is not fulfilled in the semigroup $A_{2}$, because $h\left(u_{i}\right) \neq h\left(v_{i}\right)$ implies that the sets of two-letter subwords of the words $u v$ do not coincide. Therefore, all completely 0 -simple semigroups in $V$ are orthodox $B_{2}$-semigroups (because all completely simple semigroups in $V$ are orthodox).

Thus, identity $(* *)$ is not fulfilled in some completely 0 -simple orthodox semigroup $S$. Hence, this identity is not fulfilled in some completely simple subsemigroup of $S$. We obtain a contradiction (it is easy to check that identity $(* *)$ is fulfilled in each completely simple orthodox semigroup of period $n$ ). The fact that the identity

$$
y^{2 n} x^{2 n} y^{2 n} x^{2 n} y^{2 n}=y^{2 n} x^{2 n} y^{2 n}
$$

is fulfilled in $V$ is proved similarly, because this identity is fulfilled in every orthodox completely simple semigroup of period $n$.

Two cases are possible. 
Case 1: $i=1$. Let $x$ and $y$ be two distinct letters that do not occur in the word $w_{1}$. In identity $(*)$, we substitute the word $x^{2 n} y^{2 n}$ for $h\left(u_{i}\right)$, the word $y^{2 n} x^{2 n}$ for $h\left(v_{i}\right)$, and the word $x^{2 n} y^{2 n}$ for each letter in $c\left(u_{i} w_{2} u_{2} \ldots w_{m} u_{m} w_{m+1}\right)$ distinct from $h\left(u_{i}\right)$ and $h\left(v_{i}\right)$, obtaining

$$
w_{1} x^{2 n} y^{2 n} x^{2 n} f\left(x^{2 n}, y^{2 n}\right) y^{2 n}=w_{1} y^{2 n} x^{2 n} y^{2 n} x^{2 n} g\left(x^{2 n}, y^{2 n}\right) y^{2 n} .
$$

Applying (**) and the last identity, we get

$$
w_{1} x^{2 n} y^{2 n} x^{2 n} y^{2 n}=w_{1} y^{2 n} x^{2 n} y^{2 n} x^{2 n} y^{2 n} .
$$

Applying $(* * *)$, we see that

$$
w_{1} x^{2 n} y^{2 n} x^{2 n} y^{2 n}=w_{1} y^{2 n} x^{2 n} y^{2 n},
$$

which implies the identity $z^{2 n} t x^{2 n} y^{2 n} x^{2 n} y^{2 n}=z^{2 n} t y^{2 n} x^{2 n} y^{2 n} x^{2 n} y^{2 n}$.

Case 2: $i>1$. The proof is similar to that in case 1 . It suffices to make an additional substitution: to replace all letters in $w_{1} u_{1} w_{2} u_{2} \ldots w_{i-1} u_{i-1}$ by the word $z^{2 n}$.

$(2) \Rightarrow(1)$. This follows from definitions.

The following Propositions 913 are proved similarly.

Proposition 9. Let $V \subset R S_{n}$ be a B0-Rees-Sushkevich variety. The following properties are equivalent:

(1) $V$ is a $\rho$-variety;

(2) an identity of the form

$$
x^{2 n} y^{2 n} x^{2 n} y^{2 n} t z^{2 n}=x^{2 n} y^{2 n} x^{2 n} t z^{2 n}
$$

is fulfilled in $V$.

Proposition 10. Let $V$ be a B0-Rees-Sushkevich variety. The following properties are equivalent:

(1) $V$ is a $\lambda^{*}$-variety;

(2) an identity of the form

$$
x_{1} x_{2} \ldots x_{k} x^{2 n} y^{2 n} x^{2 n} y^{2 n}=x_{1} x_{2} \ldots x_{k} y^{2 n} x^{2 n} y^{2 n}
$$

is fulfilled in $V$.

Proposition 11. Let $V$ be a B0-Rees-Sushkevich variety. The following properties are equivalent:

(1) $V$ is a $\rho^{*}$-variety;

(2) an identity of the form

$$
x^{2 n} y^{2 n} x^{2 n} y^{2 n} x_{1} x_{2} \ldots x_{k}=x^{2 n} y^{2 n} z^{2 n} x_{1} x_{2} \ldots x_{k}
$$

is fulfilled in $V$.

Proposition 12. Let $V \subset R S_{n}$ be a A0-Rees-Sushkevich variety. The following properties are equivalent:

(1) $V$ is $\bar{\lambda}$-variety;

(2) an identity of the form

$$
z^{2 n} x^{2 n} y^{2 n} x^{2 n} y^{2 n}=z^{2 n} y^{2 n} x^{2 n} y^{2 n}
$$

is fulfilled in $V$. 
Proposition 13. Let $V \subset R S_{n}$ be a B0-Rees-Sushkevich variety. The following properties are equivalent:

(1) $V$ is a $\bar{\rho}$-variety;

(2) an identity of the form

$$
x^{2 n} y^{2 n} x^{2 n} y^{2 n} t z^{2 n}=x^{2 n} y^{2 n} x^{2 n} t z^{2 n}
$$

is fulfilled in $V$.

Lemma 14. An identity $u=v$ is fulfilled in the semigroup $B_{l}\left(B_{r}\right)$ if and only if $u=v$ is a $B_{0}$-identity but is not a $\lambda$ - $(\rho)$-identity.

Proof. Assume that $u=v$ is fulfilled in the semigroup $B_{l}$. We note that the elements $g, a$, and $f$ of $B_{l}$ generate a semigroup isomorphic to $B_{0}$. Therefore, the identity $u=v$ must be fulfilled in $B_{0}$, and then Proposition 6 implies that the words $u$ and $v$ are congruent. This means that the identity $u=v$ is a $B_{0}$-identity by definition, i.e., the identity $u=v$ must have the form

$$
w_{1} u_{1} w_{2} u_{2} \ldots w_{m} u_{m} w_{m+1}=w_{1} v_{1} w_{2} v_{2} \ldots w_{m} v_{m} w_{m+1},
$$

where the $w_{i}$ are singular words, the $u_{i}$ and $v_{i}$ are words covered by cycles, and $c\left(u_{i}\right)=$ $c\left(v_{i}\right)$.

If $u=v$ is a $\lambda$-identity, then, by Proposition 8 , an identity of the form

$$
z^{2 n} t x^{2 n} y^{2 n} x^{2 n} y^{2 n}=z^{2 n} t y^{2 n} x^{2 n} y^{2 n}
$$

is fulfilled in $\operatorname{Var}\left(B_{l}\right)$. However, this identity is not fulfilled in the semigroup $B_{l}$. Indeed, replacing $z$ by $g, t$ by $a, x$ by $e$, and $y$ by $f$, we obtain the false equality gae = gaf . Therefore, $u=v$ is not a $\lambda$-identity.

Conversely, let $u=v$ be a $B_{0}$-identity but not a $\lambda$-identity. This means that $u=v$ has the form

$$
w_{1} u_{1} w_{2} u_{2} \ldots w_{m} u_{m} w_{m+1}=w_{1} v_{1} w_{2} v_{2} \ldots w_{m} v_{m} w_{m+1}
$$

where the $w_{i}$ are singular words, the $u_{i}, v_{i}$ are words covered by cycles, $c\left(u_{i}\right)=c\left(v_{i}\right)$, and $h\left(u_{i}\right)=h\left(v_{i}\right)$ for all $i$. We assign some values in $B_{l}$ to all variables in this identity (i.e., consider a map $\phi: c(u) \rightarrow B_{l}$ ).

Since $B_{l}$ is an orthodox semigroup, the set of idempotents in $B_{l}$ is a subsemigroup; moreover, the identity $x^{2} y^{2}=\left(x^{2} y^{2}\right)^{2}$ is fulfilled in $B_{l}$. By Proposition 7 the semigroup $B_{l}$ is regularly closed.

We note that the value of each word covered by cycles is a regular element of $B_{l}$ (because $B_{l}$ is a Rees-Sushkevich semigroup by Lemma 2 and is regularly closed, and the values of cyclic words in Rees-Sushkevich semigroups are regular). The regular elements of the semigroup $B_{l}$ are $0, e, f$, and $g$. We consider all possible cases.

1. $\phi\left(u_{i}\right)=0$ for some $i$. Then $\phi(u)=\phi(v)=0$.

2. $\phi\left(u_{i}\right) \neq 0$ for all $i$. Let $\phi\left(u_{i}\right)=g$ for some $i$. Then the value of each variable in the word $u_{i}$ must be equal to $g$ (because the element $g$ of the semigroup $B_{l}$ is not divisible by an element distinct from $g$ ). This means that $\phi\left(v_{i}\right)=g$.

If $\phi\left(u_{i}\right)=e$, then the value of the first letter of the word $u_{i}$ must be equal to $e$, and the values of the other variables must be either $e$ or $f$ (because the element $e$ of the semigroup $B_{l}$ is not divisible on the right by an element distinct from $e$ and $f$ ). Then $\phi\left(v_{i}\right)=e$ (because the first letters of the words $u_{i}$ and $v_{i}$ coincide).

If $\phi\left(u_{i}\right)=f$, then similar arguments show that $\phi\left(v_{i}\right)=f$. Thus, $\phi\left(u_{i}\right)=\phi\left(v_{i}\right)$ for all $i$, whence $\phi(u)=\phi(v)$.

Thus, we have proved that the identity $u=v$ is fulfilled in $B_{l}$. 
Lemma 15. An identity $u=v$ is fulfilled in the semigroup $A_{l}^{*}\left(A_{r}^{*}\right)$ if and only if $u=v$ is an $A_{0}$-identity but not a $\bar{\lambda}-(\bar{\rho})$-identity.

Proof. We proceed as in the proof of Lemma 14. It suffices to observe that the elements $g$ and $e$ of $A_{l}^{*}$ generate a subsemigroup isomorphic to $A_{0}$, and that the regular elements of the semigroup $A_{l}^{*}$ are $0, e, f$, and $g$.

Lemma 16. An identity $u=v$ is fulfilled in the semigroup $S L_{k}\left(S R_{k}\right)$ if and only if $c(u)=c(v)$ and the following two conditions are satisfied:

(1) if $u=v$ is a permutation identity, then the first (the last) $k+1$ letters of the words $u$ and $v$ coincide,

(2) if $u=v$ is not a permutation identity and $w_{u} u^{\prime}=w_{v} v^{\prime}$ is its left decomposition (respectively, $u^{\prime} w_{u}=v^{\prime} w_{v}$ is its right decomposition ), then

if the length of one of the words $w_{u}$ or $w_{v}$ is greater than $k-1$, then the length of the other word is also greater than $k-1$, and the first (the last) $k+1$ letters of the words $u$ and $v$ coincide;

if the length of one of the words $w_{u}$ or $w_{v}$ does not exceed $k$, then these words coincide and the first (the last) letters of $u^{\prime}$ and $v^{\prime}$ coincide.

Proof. The "only if" part. Let $u=v$ in the semigroup $S L_{k}$. Since $S L_{k}$ contains $Y_{2}$ as a subsemigroup, we see that $c(u)=c(v)$. Assume that $u=v$ is a permutation identity, i.e., that $u=v$ has the form $x_{1} x_{2} \ldots x_{m}=x_{\sigma 1} x_{\sigma 2} \ldots x_{\sigma m}$, where $\sigma$ is a permutation, and assume that the first $k+1$ letters of $u$ are not the same as the first $k+1$ letters of $v$. Then there is a number $q \leq k+1$ such that $\sigma i=i$ for all $i<q \leq k+1$ but $\sigma q>q$. We replace $x_{i}$ by $z_{i-q+k+1}$ for $i \leq q-1, x_{q}$ by $e, x_{\sigma q}$ by $f$, and all other variables by $e$. We prove that the resulting relation

$$
z_{k-q+2} z_{k-q+3} \ldots z_{k} e=z_{k-q+2} z_{k-q+3} \ldots z_{k} f
$$

is not valid in $S L_{k}$. Indeed, assume that this equality is fulfilled in $S L_{k}$ and apply the homomorphism $\gamma: S L_{k} \rightarrow S$ constructed in the proof of Lemma 5 to this identity (we recall that $S$ is a Rees matrix combinatorial semigroup with a square sandwich matrix of order $k+3)$. We obtain a false relation, because

$$
\gamma\left(z_{k-q+2} z_{k-q+3} \ldots z_{k} e\right)=(k-q+2, k+2) \neq 0=\gamma\left(z_{k-q+2} z_{k-q+3} \ldots z_{k} f\right) .
$$

Thus, the assumption that the first $k+1$ letters of $u$ do not coincide with the first $k+1$ letters of $v$ leads to a contradiction. Therefore, condition (1) is fulfilled.

We prove that condition (2) is also fulfilled. Let $u=v$ be a nonpermutation identity, and let its left decomposition have the form $w_{u} u^{\prime}=w_{v} v^{\prime}$. We assume that the length $m$ of the word $w_{u}=x_{1} x_{2} \ldots x_{m}$ is greater than $k-1$ and prove that the length of the other word $w_{v}$ is also greater than $k-1$. Assuming the contrary, let $\left|w_{v}\right| \leq k-1$. For all $i \leq k$, we replace the elements $x_{i}$ in the identity $u=v$ by $z_{i}$, and replace the other variables by e. Since $e S L_{k} z_{i}=0, z_{i} S L_{k} z_{i}=0$, and $z_{i}^{2}=0$, we see that $z_{1} z_{2} \ldots z_{k} e=z_{j_{1}} z_{j_{2}} \ldots z_{j_{q}} e$ or $z_{1} z_{2} \ldots z_{k} e=0$ in $S L_{k}$ for some $q \leq\left|w_{v}\right| \leq k-1$. We note that, by definition, we have $z_{j_{1}} z_{j_{2}} \ldots z_{j_{q}} e=0$ in $S L_{k}$ for $q \leq\left|w_{v}\right| \leq k-1$. Thus, $z_{1} z_{2} \ldots z_{k} e=0$ in $S L_{k}$, a contradiction (see the argument above). This contradiction proves that the length of the word $w_{v}$ is also greater than $k-1$.

We assume that the first $k+1$ letters of the words $u$ and $v$ do not coincide. Then there is $q \leq k+1$ such that the first $q-1$ letters of $u$ and $v$ coincide but the $q$ th letters of $u$ and $v$ are distinct. Let $u=x_{1} x_{2} \ldots x_{q-1} x u^{\#}$ and $v=x_{1} x_{2} \ldots x_{q-1} y v^{\#}$, where $x$ and $y$ are distinct. If $x$ or $y$ belongs to the set $\left\{x_{1}, x_{2}, \ldots, x_{q-1}\right\}$, then $\left|w_{u}\right| \leq k-1$ or $\left|w_{v}\right| \leq k-1$, which contradicts the condition of the case in question. A similar contradiction can be obtained from the assumption that, for $1 \leq i \leq q-1$, one of the letters $x_{i}$ occurs in $u^{\#}$ or in $v^{\#}$. 
In the identity $u=v$, we replace the letter $x_{i}$ by $z_{i-q+k+1}$ for $i \leq q-1$, the letter $x$ by $e, y$ by $f$, and the remaining variables by $e$. We obtain

$$
z_{k-q+2} z_{k-q+3} \ldots z_{k} e=z_{k-q+2} z_{k-q+3} \ldots z_{k} f
$$

which is not valid in $S L_{k}$ (see above). This contradiction proves that the first $k+1$ letters of the words $u$ and $v$ coincide.

Now, assume that the length of one of the words, say the length of $w_{u}$, does not exceed $k-1$. Then, as was shown above, the length of the other word $w_{v}$ also does not exceed $k-1$. We assume that the words $w_{u}$ and $w_{v}$ are distinct. Then there is $q \leq k-1$ such that the first $q-1$ letters of $w_{u}$ and $w_{v}$ coincide but the qth letters of $w_{u}$ and $w_{v}$ are distinct. Let $u=x_{1} x_{2} \ldots x_{q-1} x u^{\#}$ and $v=x_{1} x_{2} \ldots x_{q-1} y v^{\#}$, where the letters $x$ and $y$ are distinct. If $x$ or $y$ belongs to the set $\left\{x_{1}, x_{2}, \ldots, x_{q-1}\right\}$, then there is a letter in $\left\{x_{1}, x_{2}, \ldots, x_{q-1}\right\}$ that occurs more than once in $u$ or in $v$, i.e., can occur neither in $w_{u}$ nor in $w_{v}$ (by the definition of the left decomposition, all letters of $w_{u}$ occur in $u$ only once). This is a contradiction. The assumption that one of the letters $x_{i}$, where $1 \leq i \leq q-1$, occurs in $u^{\#}$ or in $v^{\#}$ also leads to a contradiction.

In the identity $u=v$, we replace $x_{i}$ by $z_{i-q+k+1}$ for each $i \leq q-1$, and replace $x$ by $e, y$ by $f$, and the remaining variables by $e$. Again, we obtain

$$
z_{k-q+2} z_{k-q+3} \ldots z_{k} e=z_{k-q+2} z_{k-q+3} \ldots z_{k} f
$$

which fails in $S L_{k}$. The assumption that the words $w_{u}$ and $w_{v}$ coincide and the first letters of $u^{\prime}$ and $v^{\prime}$ are distinct also leads to a contradiction. Thus, the words $w_{u}$ and $w_{v}$ coincide, and the first letters of $u^{\prime}$ and $v^{\prime}$ are the same.

The "if" part. Let $u=v$ be an identity satisfying (1), (2), and the relation $c(u)=c(v)$. We wish to prove that the identity $u=v$ is fulfilled in the semigroup $S L_{k}$. We consider several possible cases.

1) Let $u=v$ be a permutation identity. By condition (1), the identity $u=v$ has the form

$$
x_{1} x_{2} \ldots x_{k} x_{k+1} u^{\#}=x_{1} x_{2} \ldots x_{k} x_{k+1} v^{\#} .
$$

We prove that $\gamma\left(x_{1} x_{2} \ldots x_{k} x_{k+1} u^{\#}\right)=\gamma\left(x_{1} x_{2} \ldots x_{k} x_{k+1} v^{\#}\right)$ for every map $\gamma$ of the alphabet to the semigroup $S L_{k}$ (this means precisely that the identity $u=v$ is fulfilled in $\left.S L_{k}\right)$.

If $\gamma\left(x_{i}\right)=0$ for some $i \leq k+1$, then $\gamma\left(x_{i} \ldots x_{k+1} u^{\#}\right)=\gamma\left(x_{i} \ldots x_{k+1} v^{\#}\right)=0$. If $\gamma\left(x_{i}\right)$ is distinct from 0 but is divisible by $e$ (or by $f$ ), then $\gamma\left(x_{i}\right)=$ ced or $\gamma\left(x_{i}\right)=c f d$ for some $c, d \in S L_{k}$ or empty symbols. However, the relations ced $\neq 0$ and cfd $\neq 0$ can be fulfilled only if $d$ is not divisible by any element $z_{i}$. Hence, $d=e$ or $d=f$. In any case, the image $\gamma\left(x_{i}\right)$ is equal to $c e$ or to $c f$. If the image of a letter occurring in $c\left(x_{i+1} \ldots x_{k+1} u^{\#}\right)=c\left(x_{i+1} \ldots x_{k+1} v^{\#}\right)$ is divisible by one of the elements $z_{j}$, then, again, we see that $\gamma\left(x_{i} \ldots x_{k+1} u^{\#}\right)=\gamma\left(x_{i} \ldots x_{k+1} v^{\#}\right)=0$.

It remains to consider the case where the images of all letters occurring in

$$
c\left(x_{i+1} \ldots x_{k+1} u^{\#}\right)=c\left(x_{i+1} \ldots x_{k+1} v^{\#}\right)
$$

are not divisible by any $z_{j}$. This is possible only if these images are equal to $e$ or to $f$. However, the elements $\gamma\left(x_{i} \ldots x_{k+1} u^{\#}\right)$ and $\gamma\left(x_{i} \ldots x_{k+1} v^{\#}\right)$ are equal to ce (respectively, to $c f$ ), and again

$$
\gamma\left(x_{1} x_{2} \ldots x_{k} x_{k+1} u^{\#}\right)=\gamma\left(x_{1} x_{2} \ldots x_{k} x_{k+1} v^{\#}\right) .
$$

2) Now, let $u=v$ be a nonpermutation identity. By property (2), the left decomposition of this identity has the form $w_{u} u^{\prime}=w_{v} v^{\prime}$.

We consider all possible cases. 
2.1. $\left|w_{u}\right|>k-1$. By property (2), we have $\left|w_{v}\right|>k-1$, and the first $k+1$ letters of $u$ coincide with the first $k+1$ letters of $v$, i.e., the identity $u=v$ has the form $x_{1} x_{2} \ldots x_{k} x_{k+1} u^{\#}=x_{1} x_{2} \ldots x_{k} x_{k+1} v^{\#}$. Repeating the above arguments, we conclude that the identity $u=v$ is fulfilled in the semigroup $S L_{k}$.

2.2. $\left|w_{u}\right| \leq k-1$. By property (2), the left decomposition of $u=v$ has the form $w_{u} u^{\prime}=w_{v} v^{\prime}$ with $w_{u}=w_{v}$ and $h\left(u^{\prime}\right)=h\left(v^{\prime}\right)$. We fix an arbitrary map $\gamma$ of the alphabet to the semigroup $S L_{k}$. If $\gamma\left(h\left(u^{\prime}\right)\right)$ is divisible by an element $z_{i}$, then $\gamma\left(h\left(u^{\prime}\right)\right)=0$ because $z_{i} S L_{k} z_{i}=0$. Therefore, $\gamma\left(w_{u} u^{\prime}\right)=\gamma\left(w_{v} v^{\prime}\right)$. Suppose $\gamma\left(h\left(u^{\prime}\right)\right)$ is not divisible by any $z_{i}$. Then $\gamma\left(h\left(u^{\prime}\right)\right)=e\left(\right.$ or $\left.\gamma\left(h\left(u^{\prime}\right)\right)=f\right)$. If the image of a letter $y$ of the word $u^{\prime}$ different from $x=h\left(u^{\prime}\right)=h\left(v^{\prime}\right)$ is divisible by an element $z_{i}$, then $\gamma\left(w_{u} u^{\prime}\right)=\gamma\left(w_{v} v^{\prime}\right)=0$ because $\{e, f\} S L_{k} z_{i}=0$. Suppose that the image of every letter $y$ of $u^{\prime}$ different from $x$ is not divisible by any element $z_{i}$. This means that $\gamma(y) \in\{e, f\}$, whence $\gamma\left(w_{u} u^{\prime}\right)=\gamma\left(w_{v} v^{\prime}\right)=$ $\gamma\left(w_{u} h\left(u^{\prime}\right)\right)$. Thus, the identity $u=v$ is fulfilled in the semigroup $S L_{k}$. This completes the proof of the "if" part.

Lemma 17. An identity $u=v$ is fulfilled in the semigroup $S L_{k}^{*}\left(S R_{k}^{*}\right)$ if and only if $c(u)=c(v)$ and the following conditions are satisfied:

(1) if $u=v$ is a permutation identity, then the first (the last) $k$ letters of the words $u$ and $v$ coincide;

(2) if $u=v$ is a nonpermutation identity and $w_{u} u^{\prime}=w_{v} v^{\prime}$ is its left decomposition (respectively, $u^{\prime} w_{u}=v^{\prime} w_{v}$ is its right decomposition), then

if the length of one of the words $w_{u}$ or $w_{v}$ is greater than $k-1$, then the length of the other word is also greater than $k-1$ and the first (the last) $k$ letters of $u$ and $v$ coincide;

if the length of one of the words $w_{u}$ or $w_{v}$ does not exceed $k$, then these words coincide.

Proof. The "only if" part. Let $u=v$ in the semigroup $S L_{k}^{*}$. Since $S L_{k}^{*}$ contains $Y_{2}$ as a subsemigroup, we have $c(u)=c(v)$. We assume that $u=v$ is a permutation identity, i.e., $u=v$ has the form $x_{1} x_{2} \ldots x_{m}=x_{\sigma 1} x_{\sigma 2} \ldots x_{\sigma m}$, where $\sigma$ is a permutation. If the first $k$ letters in $u$ and $v$ do not coincide, then there is $q \leq k$ such that $\sigma q>q$ and $\sigma i=i$ for all $i<q$. For $i \leq q$, we replace $x_{i}$ by $z_{i-q+k+1}$ and all other variables by $e$. However, the equality

$$
z_{k-q+2} z_{k-q+3} \ldots z_{k} e=z_{k-q+2} z_{k-q+3} \ldots z_{k-1} e=0
$$

is not fulfilled in $S L_{k}^{*}$. Indeed, assume the contrary. Then, the proof of Lemma 5 shows that the equality

$$
\gamma\left(z_{k-q+2} z_{k-q+3} \ldots z_{k} e\right)=\gamma\left(z_{k-q+2} z_{k-q+3} \ldots z_{k-1} e\right)
$$

obtained from the preceding one by an application of the homomorphism $\gamma$ (described in the same proof) is also fulfilled in $S$. However, the left-hand side of the last equality is equal to $(k-q+2, k+1) \neq 0$, but the right-hand side is 0 . Thus, the first $k$ letters in the words $u$ and $v$ coincide.

Now let $u=v$ be a nonpermutation identity in $S L_{k}^{*}$, and let $w_{u} u^{\prime}=w_{v} v^{\prime}$ be its left representation. Assume that the length $m$ of one of the words, e.g., the length of the word $w_{u}=x_{1} x_{2} \ldots x_{m}$, is greater than $k-1$. We prove that the length of the other word $w_{v}$ is also greater than $k-1$. Assuming the contrary, let $\left|w_{v}\right| \leq k-1<m$. In the identity $u=v$, we replace the element $x_{i}$ by $z_{i}$ for all $i \leq k$ and substitute $e$ for the remaining elements. We see that, in $S L_{k}$, either $z_{1} z_{2} \ldots z_{k} e=z_{j_{1}} z_{j_{2}} \ldots z_{j_{q}}$ e for some $q \leq\left|w_{v}\right| \leq k-1$, or $z_{1} z_{2} \ldots z_{k} e=0$ (the latter follows from the fact that in $S L_{k}$ we have the relations $e S L_{k} z_{i}=0, z_{i} S L_{k} z_{i}=0$, and $\left.z_{i}^{2}=0\right)$. However, if $q \leq\left|w_{v}\right| \leq k-1$, then the relation $z_{j_{1}} z_{j_{2}} \ldots z_{j_{q}} e=0$ is fulfilled in $S L_{k}$. Therefore, in both cases we see that $z_{1} z_{2} \ldots z_{k} e=0$, which is false (see above). This contradiction proves that the length of the word $w_{v}$ is also greater than $k-1$. 
Assume that the first $k$ letters of the words $u$ and $v$ do not coincide. Then there is $q \leq k$ such that the first $q-1$ letters of $u$ and $v$ coincide but the $q$ th letters are distinct, i.e.,

$$
u=x_{1} x_{2} \ldots x_{q-1} x u^{\#}, \quad v=x_{1} x_{2} \ldots x_{q-1} y v^{\#},
$$

where $x$ and $y$ are distinct letters. If $x$ or $y$ belongs to the set $\left\{x_{1}, x_{2}, \ldots, x_{q-1}\right\}$, then $\left|w_{u}\right| \leq k-1$ or $\left|w_{v}\right| \leq k-1$, which contradicts the assumption. A similar contradiction can be obtained from the assumption that one of the letters $x_{i}, 1 \leq i \leq q-1$, occurs in $u^{\#}$ or $v^{\#}$. In the identity $u=v$, we replace $x_{i}$ by $z_{i-q+k}$ for each $i \leq q-1$ and substitute $z_{k}$ for $x$, and $e$ for $y$ and the remaining letters. Again, we see that $z_{k-q+1} z_{k-q+2} \ldots z_{k} e=$ $z_{k-q+1} z_{k-q+2} \ldots z_{k-1} e=0$, which, as proved above, is false in $S L_{k}^{*}$. This contradiction shows that the first $k$ letters of the words $u$ and $v$ coincide.

Now, suppose that the length of one of the words, say, the length of $w_{u}$, does not exceed $k-1$. Then, as was proved above, the length of the other word $w_{v}$ also does not exceed $k-1$. If these words do not coincide, then

$$
u=x_{1} x_{2} \ldots x_{q-1} x u^{\#}, \quad v=x_{1} x_{2} \ldots x_{q-1} y v^{\#}, \quad x \neq y,
$$

for some $q \leq k-1$. If $x$ or $y$ belongs to the set $\left\{x_{1}, x_{2}, \ldots, x_{q-1}\right\}$, then some letter in $\left\{x_{1}, x_{2}, \ldots, x_{q-1}\right\}$ occurs in $u$ or in $v$ more than once, i.e., this letter can occur neither in the word $w_{u}$, nor in the word $w_{v}$ (by the definition of the left decomposition, all letters of $w_{u}$ occur in the word $u$ only once). A similar contradiction can be obtained from the assumption that one of the letters $x_{i}, 1 \leq i \leq q-1$, occurs in $u^{\#}$ or $v^{\#}$. In the identity $u=v$, we replace $x_{i}$ by $z_{i-q+k}$ for each $i \leq q-1$, and substitute $e$ for $x, f$ for $y$, and $e$ for the remaining variables. Again, we arrive at the equality $z_{k-q+1} z_{k-q+2} \ldots z_{k} e=z_{k-q+1} z_{k-q+2} \ldots z_{k-1} e=0$, which is false in $S L_{k}^{*}$. A similar contradiction can be obtained from the assumption that the words $w_{u}$ and $w_{v}$ coincide and the first letters of $u^{\prime}$ and $v^{\prime}$ are distinct. Thus, the words $w_{u}$ and $w_{v}$ coincide, and the first letters of $u^{\prime}$ and $v^{\prime}$ coincide.

The "if" part. Let $c(u)=c(v)$, and let conditions (1) and (2) be fulfilled. We prove that the identity $u=v$ is fulfilled in the semigroup $S L_{k}^{*}$. We consider all possible cases.

1) Let $u=v$ be a permutation identity. By condition (1), the identity $u=v$ has the form $x_{1} x_{2} \ldots x_{k} u^{\#}=x_{1} x_{2} \ldots x_{k} v^{\#}$. We prove that

$$
\gamma\left(x_{1} x_{2} \ldots x_{k} x_{k+1} u^{\#}\right)=\gamma\left(x_{1} x_{2} \ldots x_{k} x_{k+1} v^{\#}\right)
$$

for an arbitrary map $\gamma$ of the alphabet to the semigroup $S L_{k}^{*}$ (this means that the identity $u=v$ is fulfilled in $\left.S L_{k}^{*}\right)$.

If $\gamma\left(x_{i}\right)$ is divisible by $e$, then $\gamma\left(x_{i}\right)=$ ced for some elements $c, d \in S L_{k}^{*}$ or empty symbols. If $\gamma\left(x_{i}\right)=0$, then $\gamma\left(x_{i} \ldots x_{k} u^{\#}\right)=\gamma\left(x_{i} \ldots x_{k} v^{\#}\right)=0$. Let $\gamma\left(x_{i}\right) \neq 0$. This means that $d$ is not divisible by any element $z_{i}$. Therefore, $d=e$ and $\gamma\left(x_{i}\right)=c e$. If the image of some letter in

$$
c\left(x_{i+1} \ldots x_{k} u^{\#}\right)=c\left(x_{i+1} \ldots x_{k} v^{\#}\right)
$$

is divisible by $z_{i}$, then $\gamma\left(x_{i} \ldots x_{k} u^{\#}\right)=\gamma\left(x_{i} \ldots x_{k} v^{\#}\right)=0$. If the images of all letters in $c\left(x_{i+1} \ldots x_{k} u^{\#}\right)=c\left(x_{i+1} \ldots x_{k} v^{\#}\right)$ are not divisible by $z_{i}$, then these images can be equal only to $e$. Thus, $\gamma\left(x_{i} \ldots x_{k} u^{\#}\right)=\gamma\left(x_{i} \ldots x_{k} v^{\#}\right)=c e$. Hence, $\gamma\left(x_{1} x_{2} \ldots x_{k} u^{\#}\right)=$ $\gamma\left(x_{1} x_{2} \ldots x_{k} v^{\#}\right)$.

2) Let $u=v$ be a nonpermutation identity the left decomposition $w_{u} u^{\prime}=w_{v} v^{\prime}$ of which satisfies (2). We consider all possible cases.

2.1. $\left|w_{u}\right|>k-1$. Then, by property (2), we obtain $\left|w_{u}\right|>k-1$, and the first $k$ letters of $u$ coincide with the first $k$ letters of $v$, i.e., the identity $u=v$ has the form $x_{1} x_{2} \ldots x_{k} u^{\#}=x_{1} x_{2} \ldots x_{k} v^{\#}$. Repeating the above argument, we conclude that the identity $u=v$ is fulfilled in the semigroup $S L_{k}^{*}$. 
2.2. $\left|w_{u}\right| \leq k-1$. Then, by property (2), we have $w_{u}=w_{v}=w$, so that $c\left(u^{\prime}\right)=c\left(v^{\prime}\right)$. Let $\gamma$ be an arbitrary map of the alphabet to the semigroup $S L_{k}$.

2.2.1. Let $\gamma(w)$ be divisible by $e$ in the semigroup $S L_{k}^{*}$. If the $\gamma$-image of some letter in $c\left(u^{\prime}\right)=c\left(v^{\prime}\right)$ is divisible by some $z_{i}$, then $\gamma\left(w_{u} u^{\prime}\right)=\gamma\left(w_{v} v^{\prime}\right)$ because $z_{i} S L_{k} z_{i}=0$.

Suppose the $\gamma$-images of all letters in $c\left(u^{\prime}\right)=c\left(v^{\prime}\right)$ are not divisible by any element $z_{i}$. This means that the images of all such letters are equal to $e$. Therefore, $\gamma\left(u^{\prime}\right)=\gamma\left(v^{\prime}\right)=e$, whence $\gamma\left(w_{u} u^{\prime}\right)=\gamma\left(w_{v} v^{\prime}\right)$.

2.2.2. Let $\gamma(w)$ be not divisible by $e$ in the semigroup $S L_{k}^{*}$. If $\gamma(w)=0$, then $\gamma\left(w_{u} u^{\prime}\right)=\gamma\left(w_{v} v^{\prime}\right)=0$. Now let $\gamma(w) \neq 0$. This means that $\gamma(w)=z_{i} z_{i+1} \ldots z_{j}$ for some $i \leq j$. We assume that $j<k$ and consider all possible cases.

a) $\gamma\left(h\left(u^{\prime}\right)\right) \neq e$ and $\gamma\left(h\left(v^{\prime}\right)\right) \neq e$. This means that each of the elements $\gamma\left(h\left(u^{\prime}\right)\right)$ and $\gamma\left(h\left(v^{\prime}\right)\right)$ is divisible by some $z_{i}$. Since $z_{i} S L_{k} z_{i}=0$, we see that $\gamma\left(h\left(u^{\prime}\right)\right)=0$ and $\gamma\left(h\left(v^{\prime}\right)\right)=0$. It follows that $\gamma\left(w_{u} u^{\prime}\right)=\gamma\left(w_{v} v^{\prime}\right)$. (We have used the fact that, by the definition of the left decomposition, $z_{i} S L_{k} z_{i}=0$ and the first letters of the words $u^{\prime}$ and $v^{\prime}$ occur in these words more than once.)

b) $\gamma\left(h\left(u^{\prime}\right)\right)=e$ and $\gamma\left(h\left(v^{\prime}\right)\right) \neq e$. This means that $\gamma\left(h\left(v^{\prime}\right)\right)$ is divisible by some $z_{i}$, whence $\gamma\left(u^{\prime}\right)=0$. Since in this case we have $\gamma\left(u^{\prime}\right) \in e S L_{k} z_{i} S_{l, k}^{1}=0$, again we conclude that $\gamma\left(w_{u} u^{\prime}\right)=\gamma\left(w_{v} v^{\prime}\right)$.

c) $\gamma\left(h\left(u^{\prime}\right)\right) \neq e$ and $\gamma\left(h\left(v^{\prime}\right)\right)=e$. This case can be treated like the previous one.

d) $\gamma\left(h\left(u^{\prime}\right)\right)=e$ and $\gamma\left(h\left(v^{\prime}\right)\right)=e$. If the image of a letter $y$ in $c\left(u^{\prime}\right)=c\left(v^{\prime}\right)$ is divisible by some $z_{i}$, then $\gamma\left(w_{u} u^{\prime}\right)=\gamma\left(w_{v} v^{\prime}\right)=0$ because $e S L_{k} z_{i}=0$. If the images of all letters in $c\left(u^{\prime}\right)=c\left(v^{\prime}\right)$ are not divisible by any element $z_{i}$, then all these images are equal to $e$, whence $\gamma\left(u^{\prime}\right)=\gamma\left(v^{\prime}\right)=e$. It follows that $\gamma\left(w_{u} u^{\prime}\right)=\gamma\left(w_{v} v^{\prime}\right)$.

Lemma 18. Let $V$ be a Rees-Sushkevich variety, and let $u$ and $v$ be regular words. If the identity $u=v$ is fulfilled in all completely 0 -simple semigroups in $V$, then this identity is also fulfilled in the entire $V$.

Proof. This follows from Lemma 4 on regular elements. It suffices to observe that, in the Rees-Sushkevich semigroups, the values of regular words are regular elements (see [9]).

Proposition 19. A Rees-Sushkevich variety $V$ is diverse if and only if $C N_{k} \notin V$ for some $k$, i.e., if $n_{D}(V) \neq \infty$.

Proof. This follows from Lemma 3 and the definitions.

Proposition 20. A Rees-Sushkevich B0-variety $V$ is a $\lambda$ - $(\rho)$-variety if and only if $B_{l} \notin V\left(B_{r} \notin V\right)$.

Proof. This follows from Lemma 14 and the definitions.

Proposition 21. A Rees-Sushkevich A0-variety $V$ is a $\bar{\lambda}$ - $(\bar{\rho})$-variety if and only if $A_{l}^{*} \notin V\left(A_{r}^{*} \notin V\right)$.

Proof. This follows from Lemma 15 and the definitions.

Proposition 22. A Rees-Sushkevich B0-variety $V$ is a $\lambda^{*}-\left(\rho^{*}\right)$-variety if and only if $S L_{k} \notin V\left(S R_{k} \notin V\right)$ for all $k$.

Proof. This follows from Lemma 16 and the definitions.

Proposition 23. Let $V \subset R S_{n}$ be a Rees-Sushkevich variety containing the semigroup $Y_{2}$. The following conditions are equivalent:

(1) $V$ does not contain the semigroup $S L_{k}\left(S R_{k}\right)$; 
(2) one of the identities

$$
\begin{aligned}
& q_{l, k}: x_{1} \ldots x_{k} x^{2 n} y^{2 n} x^{2 n} y^{2 n}=x_{1} \ldots x_{k} y^{2 n} x^{2 n} y^{2 n} \\
& q_{l, k}^{*}: x_{1} \ldots x_{k-1} x_{k} y^{2}=x_{1} \ldots x_{k-1} x_{k}^{n+1} y^{2}
\end{aligned}
$$

(respectively, the identities

$$
\begin{aligned}
& q_{r, k}: x^{2 n} y^{2 n} x^{2 n} y^{2 n} x_{1} \ldots x_{k}=x^{2 n} y^{2 n} x^{2 n} x_{1} \ldots x_{k}, \\
& \left.q_{r, k}^{*}: y^{2} x_{1} x_{2} \ldots x_{k}=y^{2} x_{1}^{n+1} x_{2} \ldots x_{k}\right)
\end{aligned}
$$

is fulfilled in $V$.

Proof. (1) $\Rightarrow(2)$. Let $V \subset R S_{n}$ be a variety of semigroups. We assume that $V$ contains $Y_{2}$ and does not contain $S L_{k}$. This means that there is an identity $u=v$ valid in $V$ and false in $S L_{k}$. Since $Y_{2} \in V$, we have $c(u)=c(v)$. Let $w_{u} u^{\prime}=w_{v} v^{\prime}$ be the left decomposition of $u=v$. Since the identity $u=v$ is not fulfilled in $S L_{k}$, Lemma 16 implies the existence of $q \leq k+1$ such that the first $q-1$ letters of the words $u$ and $v$ coincide and the $q$ th letters are distinct. We consider all possible cases.

1.1. $u=v$ is a permutation identity. Then

$$
u=x_{1} x_{2} \ldots x_{q-1} x u^{\#}, \quad v=x_{1} x_{2} \ldots x_{q-1} y v^{\#},
$$

where $x_{1}, x_{2}, \ldots, x_{q-1}, x, y$ are different letters and the letters $x_{1}, x_{2}, \ldots, x_{q-1}$ are distinct from the letters of the words $u^{\#}$ and $v^{\#}$. The fact that the identity $u=v$ is fulfilled in the variety $V$ implies that the semigroup $A_{2}$ and all semigroups $M_{p}$ do not belong to $V$. Consequently, all completely 0 -simple semigroups in $V$ are orthodox. In the identity $u=v$, we replace $x$ by $x^{2 n}, y$ by $y^{2 n}$, and the remaining letters distinct from $x_{1}, x_{2}, \ldots, x_{q-1}, x$, and $y$ by $x^{2 n}$. Multiplying both sides of the resulting relation from the right by $x^{2 n} y^{2 n}$, we obtain an identity of the form

$$
x_{1} \ldots x_{q-1} x^{2 n} P\left(x^{2 n}, y^{2 n}\right) x^{2 n} y^{2 n}=x_{1} \ldots x_{q-1} y^{2 n} Q\left(x^{2 n}, y^{2 n}\right) x^{2 n} y^{2 n} .
$$

Next, since all 0-simple semigroups in $V$ are orthodox, the identities

$$
\begin{aligned}
& x^{2 n} P\left(x^{2 n}, y^{2 n}\right) x^{2 n} y^{2 n}=x^{2 n} y^{2 n} x^{2 n} y^{2 n}, \\
& y^{2 n} Q\left(x^{2 n}, y^{2 n}\right) x^{2 n} y^{2 n}=y^{2 n} x^{2 n} y^{2 n}
\end{aligned}
$$

are fulfilled in these semigroups. The words occurring on the two sides of these identities are regular. Therefore, by Lemma 18, the above identities are fulfilled in the variety $V$. Therefore, $(*)$ implies the identity $q_{l, q-1}$, and consequently, since $q \leq k+1$, the identity $q_{l, k}$.

1.2. $u=v$ is not a permutation identity.

1.2.1. Let $\left|w_{u}\right| \leq k-1<\left|w_{v}\right|$. Then $c\left(u^{\prime}\right) \cap c\left(w_{v}\right) \neq \varnothing$ because $c(u)=c(v)$, and the identity $u=v$ has the form

$$
u_{0} x u_{1} x u_{2}=x_{q} x_{2} \ldots x_{k-1} x v^{\#}
$$

for some $1 \leq q<k$ and different letters $x \notin c\left(u_{0}\right), x_{q}, x_{2}, \ldots, x_{k-1}$ distinct from the letters of the word $v^{\#}$. Replacing $x$ by $x y^{2 n}$ and all letters of the word $v^{\#}$ by $y^{2 n}$, we obtain the identity

$$
x_{q} \ldots x_{k-1} x y^{2 n}=Q, \quad Q=\bar{u}_{0} x y^{2 n} \bar{u}_{1} x y^{2 n} \bar{u}_{2} y^{2 n},
$$

which fails in the semigroup $B_{2}$ (it suffices to make the substitution $x_{i} \rightarrow(1,1), x \rightarrow$ $(1,2), y \rightarrow(2,2))$. Therefore, $B_{2} \notin V$, and thus, all 0 -simple semigroups in $V$ are completely simple, with, possibly, a zero adjoined. In such semigroups the identity $x=x^{n+1}$ is fulfilled. Therefore, the identity $Q=\widetilde{Q}$, where $\widetilde{Q}$ is the word obtained from $Q$ by the replacement of the letter $x$ by $x^{n+1}$ is also fulfilled. Since both sides of the identity $Q=\widetilde{Q}$ are regular words, Lemma 18 implies that this identity is fulfilled in $V$. From 
$Q=\widetilde{Q}$ and identity $(*)$, we obtain the identity $x_{q} \ldots x_{k-1} x y^{2 n}=\widetilde{Q}$. On the other hand, replacing $x$ by $x^{n+1}$ in the identity $x_{q} \ldots x_{k-1} x y^{2 n}=Q$, we obtain $x_{q} \ldots x_{k-1} x^{n+1} y^{2 n}=$ $\widetilde{Q}$. The last two identities show that $x_{q} \ldots x_{k-1} x y^{2 n}=x_{q} \ldots x_{k-1} x^{n+1} y^{2 n}$. Replacing $x$ by $x_{k}$, multiplying from the left by $x_{1} \ldots x_{q-1}$ and from the right by $y^{2}$, and using the fact that the relation $y^{2}=y^{n+2}=y^{2 n+2}$ is fulfilled in the variety $R S_{n}$, we obtain the identity $q_{l, k}^{*}: x_{1} x_{2} \ldots x_{k} y^{2}=x_{1} x_{2} \ldots x_{k}^{n+1} y^{2}$.

1.2.2. Suppose $\left|w_{u}\right|,\left|w_{v}\right| \leq k-1$ and $w_{u} \not \equiv w_{v}$ or $h\left(u^{\prime}\right) \neq h\left(v^{\prime}\right)$. This means that the identity $u=v$ has the form

$$
x_{1} x_{2} \ldots x_{q-1} x u^{\#}=x_{1} x_{2} \ldots x_{q-1} y v^{\#},
$$

where $1 \leq q \leq k-1$, the letters $x_{1}, x_{2}, \ldots, x_{q-1}, x, y$ are distinct, and the letters $x_{1}, x_{2}, \ldots, x_{q-1}$ are distinct from the letters of the words $u^{\#}$ and $v^{\#}$. As was shown in Subcase 1.1, this identity implies the identity $q_{l, k}$.

1.2.3. If $\left|w_{u}\right|,\left|w_{v}\right| \leq k-1, w_{u} \equiv w_{v}$, and $h\left(u^{\prime}\right)=h\left(v^{\prime}\right)$, then Lemma 16 shows that the identity $u=v$ is fulfilled in the semigroup $S L_{k}$, which contradicts the assumption.

1.2.4. Finally, let $\left|w_{u}\right|,\left|w_{v}\right|>k-1$. If the first $k+1$ letters of the words $u$ and $v$ coincide, then $u=v$ in the semigroup $S L_{k}$ by Lemma 16, which contradicts the assumption. Consequently, the first $k+1$ letters of the words $u$ and $v$ cannot coincide in this case. Suppose the first $q-1$ letters of the words $u$ and $v$ coincide but the $q$ th letters are distinct for some $q \leq k+1$. Then $u=x_{1} x_{2} \ldots x_{q-1} x u^{\#}$ and $v=x_{1} x_{2} \ldots x_{q-1} y v^{\#}$, where $x_{1}, x_{2}, \ldots, x_{q-1}, x$, and $y$ are different letters. The word $x_{1} x_{2} \ldots x_{q-1}$ is an initial subword of the words $w_{u}$ and $w_{v}$. Therefore, the lengths of $w_{u}$ and $w_{v}$ are at least $k \geq q-1$. Consequently, the letters $x_{1}, x_{2}, \ldots, x_{q-1}$ are distinct from the letters of the words $u^{\#}$ and $v^{\#}$. As was shown in Subcase 1.1, such an identity $u=v$ implies the identity $q_{l, k}$.

$(1) \Rightarrow(2)$. This follows immediately from Lemma 16 .

Proposition 24. Let $V \subset R S_{n}$ be a Rees-Sushkevich variety containing the semigroup $Y_{2}$. The following conditions are equivalent:

(1) $V$ does not contain the semigroup $S L_{k}^{*}\left(S R_{k}^{*}\right)$;

(2) the identity $q_{l, k}^{*}: x_{1} x_{2} \ldots x_{k} y^{2}=x_{1} x_{2} \ldots x_{k}^{n+1} y^{2}$ (respectively, the identity $q_{r, k}^{*}$ : $\left.y^{2} x_{1} x_{2} \ldots x_{k}=y^{2} x_{1}^{n+1} x_{2} \ldots x_{k}\right)$ is fulfilled in $V$.

Proof. (1) $\Rightarrow(2)$. Let $V \subset R S_{n}$ be a variety of semigroups that contains $Y_{2}$ and does not contain $S L_{k}^{*}$. This means that there is an identity $u=v$ that is fulfilled in $V$ and is not fulfilled in $S L_{k}^{*}$. Since $Y_{2} \in V$, we have $c(u)=c(v)$. Let $w_{u} u^{\prime}=w_{v} v^{\prime}$ be the left decomposition of the identity $u=v$. We consider all possible cases.

1.1. $u=v$ is a permutation identity.

Since $u=v$ is not fulfilled in $S L_{k}^{*}$, Lemma 17 implies that there is $q \leq k$ such that the first $q-1$ letters of the words $u$ and $v$ coincide but the $q$ th letters are distinct. Then

$$
u=x_{1} x_{2} \ldots x_{q-1} x u^{\#}, \quad v=x_{1} x_{2} \ldots x_{q-1} y v^{\#},
$$

where $x_{1}, x_{2}, \ldots, x_{q-1}, x$, and $y$ are different letters, the letters $x_{1}, x_{2}, \ldots, x_{q-1}$ differ from the letters of the words $u^{\#}$ and $v^{\#}$, and $x \notin c\left(u^{\#}\right), x \in c\left(v^{\#}\right)$. Since the identity $u=v$ is fulfilled in $V$, the semigroup $A_{2}$ and all subgroups $M_{p}$ do not belong to $V$. Hence, all completely 0 -simple semigroups in $V$ are orthodox.

In the identity $u=v$, we replace all letters distinct from $x_{1}, x_{2}, \ldots, x_{q-1}$, and $x$ by $y^{2 n}$ and multiply both sides of the resulting relation by $y^{2 n}$ from the right. We obtain an identity of the form

$$
x_{1} \ldots x_{q-1} x y^{2 n}=x_{1} \ldots x_{q-1} y^{2 n} Q\left(x, y^{2 n}\right) x y^{2 n}
$$


(without loss of generality, we may assume that the letter $x$ occurs on the right-hand side of the this identity more than once; this can be ensured by application of the identity $\left.\left(\mathrm{II}_{n}\right)\right)$. As was shown in Subcase 1.2.1 of the proof of Proposition 23, the abovementioned identity implies the identity $q_{l, k}^{*}$.

1.2. $u=v$ is not a permutation identity.

1.2.1. Let $\left|w_{u}\right| \leq k-1<\left|w_{v}\right|$. Since $\left.c(u)=c(v)\right)$, we see that $c\left(u^{\prime}\right) \cap c\left(w_{v}\right) \neq \varnothing$, so that the identity $u=v$ has the form $u_{0} x u_{1} x u_{2}=x_{1} x_{2} \ldots x_{q-1} x v^{\#}$, where $1 \leq q \leq k$, $x, x_{1}, x_{2}, \ldots, x_{q-1}$ are different letters distinct from the letters of the word $v^{\#}$, and $x \notin$ $c\left(u_{0}\right)$. As was shown in Subcase 1.2.1 of the proof of Proposition 23, this implies the identity $q_{l, k}^{*}$.

1.2.2. Suppose $\left|w_{u}\right|,\left|w_{v}\right| \leq k-1$ and $w_{u} \not \equiv w_{v}$. This means that the identity $u=v$ has the form

$$
x_{1} x_{2} \ldots x_{q-1} x u^{\#}=x_{1} x_{2} \ldots x_{q-1} y v^{\#},
$$

where $0 \leq q \leq k, x_{1}, x_{2}, \ldots, x_{q-1}, x$, and $y$ are different letters, the letters $x_{1}, x_{2}, \ldots, x_{q-1}$ differ from the letters of the words $u^{\#}$ and $v^{\#}, x \notin c\left(u^{\#}\right)$, and $x \in c\left(v^{\#}\right)$. As shown in Subcase 1.1 of the proof of the present proposition, this implies the identity $q_{l, k}^{*}$.

1.2.3. If $\left|w_{u}\right|,\left|w_{v}\right| \leq k-1$ and $w_{u} \equiv w_{v}$, then Lemma 17 implies that the identity $u=v$ is fulfilled in the semigroup $S L_{k}^{*}$, which contradicts the assumption.

1.2.4. Let $\left|w_{u}\right|,\left|w_{v}\right|>k-1$. If the first $k$ letters of the words $u$ and $v$ coincide, then Lemma 17 implies that the identity $u=v$ is fulfilled in the semigroup $S L_{k}^{*}$, which contradicts the assumption. Consequently, for some $q \leq k$ we have

$$
u=x_{1} x_{2} \ldots x_{q-1} x u^{\#}, \quad v=x_{1} x_{2} \ldots x_{q-1} y v^{\#},
$$

where $x_{1}, x_{2}, \ldots, x_{q-1}, x, y$ are different letters, the letters $x_{1}, x_{2}, \ldots, x_{q-1}$ differ from the letters of the words $u^{\#}$ and $v^{\#}, x \notin c\left(u^{\#}\right)$, and $x \in c\left(v^{\#}\right)$. As was shown in Subcase 1.1 of the proof of the present proposition, this implies the identity $q_{l, k}^{*}$.

The implication $(1) \Rightarrow(2)$ follows immediately from Lemma 17

Lemma 25. Let $\mathfrak{R}(V) \neq 0$. Then $\mathfrak{R}(V)=V \cap\left[x^{2}=0, x y x=0\right]$.

Proof. Let $\mathfrak{R}(V) \neq 0$. First, we prove that $\mathfrak{R}(V) \subseteq\left(V \cap\left[x^{2}=0, x y x=0\right]\right)$, i.e., that every identity $u=v$ valid in $V \cap\left[x^{2}=0, x y x=0\right]$ is also fulfilled in $\mathfrak{R}(V)$. We consider all possible cases.

1 ) Let $u$ and $v$ be nonsingular words. In this case, the identity $u=v$ follows from the identities $x^{2}=0$ and $x y x=0$; therefore, this identity is fulfilled in $\mathfrak{R}(V)$.

2) Let $u$ be singular and $v$ nonsingular. Then $|u| \geq n_{D}$, and therefore, the identities $u=0$ and $v=0$ are fulfilled in $\mathfrak{R}(V)$. Consequently, the identity $u=v$ is also fulfilled in $\mathfrak{R}(V)$.

3 ) Let $u$ be nonsingular and $v$ singular. This case is similar to the preceding one.

4) Let the words $u$ and $v$ be singular. If $u=v$ is a nonpermutation identity, then $|u| \geq n_{D}$ and $|v| \geq n_{D}$, and the identities $u=0$ and $v=0$ are fulfilled in $\mathfrak{R}(V)$. Therefore, the identity $u=v$ is also fulfilled in $\mathfrak{R}(V)$. If $u=v$ is a nonpermutation identity and $|u|<n_{D}$, then the identity $u=v$ is fulfilled in $\mathfrak{R}(V)$ by definition. If $u=v$ is a permutation identity and $|u| \geq n_{D}$, then the identities $u=0$ and $v=0$ are fulfilled in $\mathfrak{R}(V)$. Therefore, the identity $u=v$ is also fulfilled in $\mathfrak{R}(V)$.

Now we prove that $\mathfrak{R}(V) \supseteq\left(V \cap\left[x^{2}=0, x y x=0\right]\right)$, i.e., that every identity $u=v$ valid in $\mathfrak{R}(V)$ is also fulfilled in $V \cap\left[x^{2}=0, x y x=0\right]$. We consider all possible cases.

1) If $u$ and $v$ are nonsingular words, then the identity $u=v$ follows from $x^{2}=0$; $x y x=0$, and, therefore, the identity $u=v$ is fulfilled in $V \cap\left[x^{2}=0, x y x=0\right]$.

2) Suppose the word $u$ is singular, and the word $v$ is nonsingular. If $|u|<n_{D}$, none of the transformations of a basis of identities in $\mathfrak{R}(V)$ can be applied to the word $u$. 
This means that the identity $u=v$ does not follow from the defining identities of the variety $\mathfrak{R}(V)$. Therefore, the identity $u=v$ cannot be fulfilled in $\mathfrak{R}(V)$. Hence, we have $|u| \geq n_{D}$, and the identities $u=0$ and $v=0$ are fulfilled in $V \cap\left[x^{2}=0, x y x=0\right]$, whence we see that the identity $u=v$ is also fulfilled in $V \cap\left[x^{2}=0, x y x=0\right]$.

3) The case where $u$ is a nonsingular word and $v$ is a singular word is similar to the preceding one.

4) Let the words $u$ and $v$ be singular. If $u=v$ is a nonpermutation identity, then $|u| \geq n_{D}$ and $|v| \geq n_{D}$. Therefore, the identities $u=0$ and $v=0$ are fulfilled in $V \cap\left[x^{2}=0, x y x=0\right]$. If $u=v$ is a permutation identity and $|u|<n_{D}$, then the identity $u=v$ must be fulfilled in $V$. Thus, $u=v$ in $V \cap\left[x^{2}=0, x y x=0\right]$ by definition. Finally, if $u=v$ is a permutation identity and $|u| \geq n_{D}$, then $u=0$ and $v=0$ in $V \cap\left[x^{2}=0, x y x=0\right]$. Therefore, the identity $u=v$ is also fulfilled in $V \cap\left[x^{2}=0, x y x=0\right]$.

Lemma 26. If a permutation identity $x_{1} x_{2} \ldots x_{k}=x_{\sigma 1} x_{\sigma 2} \ldots x_{\sigma k}$ is fulfilled in a completely 0 -simple semigroup $S$ of period $n$, then the identity $x_{1} x_{2} \ldots x_{k}=\left(x_{1} x_{2} \ldots x_{k}\right)^{n+1}$ is also fulfilled in $S$.

Proof. Let

$$
x_{1} x_{2} \ldots x_{k}=x_{\sigma 1} x_{\sigma 2} \ldots x_{\sigma k}
$$

be a permutation identity fulfilled in a completely 0 -simple semigroup $S$ of period $n$. By Proposition 6, we have $B_{0} \notin \operatorname{Var}(S)$. Therefore, $B_{2} \notin \operatorname{Var}(S)$. This means that $S$ is a completely simple semigroup, possibly, with zero adjoined. Therefore, the identity $z=z^{n+1}$ is fulfilled in $S$, which implies that the identity $x_{1} x_{2} \ldots x_{k}=\left(x_{1} x_{2} \ldots x_{k}\right)^{n+1}$ is fulfilled in $S$.

Lemma 27. If an identity $u=v$ is not fulfilled in a completely 0 -simple semigroup $K$, then the identity $u^{f}=v^{f}$ obtained from $u=v$ by replacing every letter $x$ that occurs in $u=v$ by the word $x y_{x} t z_{x} x$ also fails in $K$. Here, $t, y_{x}$, and $z_{x}$ are the letters that do not occur in $u$ and $v$.

Proof. It suffices to observe that, for arbitrary elements $x$ and $t$ of a completely 0 -simple semigroup $K(t \neq 0)$, the equation $x=x y_{x} t z_{x} x$ has a solution.

Lemma 28. Let $V \subset R S_{n}$ be a Rees-Sushkevich variety, and let $\left\{u_{i}=v_{i} \mid i\right\}$ be a basis of identities of $V^{\prime}$. Then the identities $\left\{u_{i}^{f}=v_{i}^{f} \mid i\right\}$ and the identities $\left(\mathrm{I}_{n}\right),\left(\mathrm{II}_{n}\right)$, and $\left(\mathrm{III}_{n}\right)$ form a basis of the set Eqreg $(V)$ of the identities $u=v$ of $V$ in which both sides $u$ and $v$ are regular.

Proof. Since the identities $\left\{u_{i}^{f}=v_{i}^{f} \mid i\right\}$ follow from $\left\{u_{i}=v_{i} \mid i\right\}$, they are fulfilled in $V^{\prime}$; we prove that the identities $\left\{u_{i}^{f}=v_{i}^{f} \mid i\right\}$ are fulfilled in $V$. Indeed, suppose that the identity $u_{i}^{f}=v_{i}^{f}$ is not fulfilled in some semigroup $S$ in $V$, i.e., we have $g\left(u_{i}^{f}\right) \neq g\left(v_{i}^{f}\right)$ for some map $g$ of the alphabet to $S$. The words $u_{i}^{f}$ and $v_{i}^{f}$ and their values in the ReesSushkevich semigroups are regular elements (see [9]). By Lemma 4 on regular elements, there is an epimorphism of the semigroup $S$ to a completely 0-somple principal factor $K$ such that $h\left(g\left(u_{i}^{f}\right)\right) \neq h\left(g\left(v_{i}^{f}\right)\right)$. This means that the identity $u_{i}^{f}=v_{i}^{f}$ is not fulfilled in the semigroup $K$, and consequently, is not fulfilled in $V^{\prime}$. This contradiction shows that all identities $u_{i}^{f}=v_{i}^{f}$ are fulfilled in the variety $V$.

Suppose that the lemma is false, i.e., an identity $u=v$, where $u$ and $v$ are regular, is fulfilled in $V$ and does not follow from the identities $\left\{u_{i}^{f}=v_{i}^{f} \mid i\right\}$, (I), (II), (III). This means that there is a semigroup $S$ in which the identities $\left\{u_{i}^{f}=v_{i}^{f} \mid i\right\}$, (I), (II), and (III) are fulfilled, but the identity $u=v$ is not. Then there is a map $g$ of the alphabet to the semigroup $S$ such that $g(u) \neq g(v)$. However, $S$ is a Rees-Sushkevich semigroup. 
Therefore, the values of regular words must be regular elements. By Lemma 4 on regular elements, there is an epimorphism $h$ of $S$ to a completely 0 -simple principal factor $K$ such that $h(g(u)) \neq h(g(v))$. This means that the identity $u=v$ is not fulfilled in the semigroup $K$. We note that all identities $\left\{u_{i}=v_{i} \mid i\right\}$ are fulfilled in $K$, because otherwise, by Lemma 27, some of the identities $\left\{u_{i}^{f}=v_{i}^{f} \mid i\right\}$ are not fulfilled in $K$ and, consequently, in $S$. This means that the semigroup $K$ belongs to $V^{\prime}$, because it satisfies all identities in the basis identities of this variety. However, $V^{\prime}$ is a subvariety of $V$, whence $K$ belongs to $V$. Therefore, all identities in $E q(V)$ are fulfilled in $K$. Consequently, all identities in $\operatorname{Eqreg}(V)$, including the identity $u=v$, are fulfilled in $K$, which proves the lemma.

Lemma 29. If the identity $x_{1} x_{2} \ldots x_{k}=w$, where $|w|>k$, is fulfilled in a variety of semigroups of period $n$, then, for some integers $l, r \geq 0$ and $m>0$ such that $l+m+r=k$, the identity

$$
x_{1} \ldots x_{l} y_{1} \ldots y_{m} z_{1} \ldots z_{r}=x_{1} \ldots x_{l}\left(y_{1} \ldots y_{m}\right)^{n+1} z_{1} \ldots z_{r}
$$

is fulfilled in $V$.

Proof. If some letters except for $x_{1}, \ldots, x_{k}$ occur in the word $w$, then we replace all such letters by $x_{1}$. If, on the contrary, a letter $x_{i}$ does not occur in $w$, then the substitution $x_{i} \rightarrow x_{i}^{n+1}$ yields the required identity $x_{1} \ldots x_{i} \ldots x_{k}=w=x_{1} \ldots x_{i}^{n+1} \ldots x_{k}$. Therefore, we may assume that all letters $x_{1}, \ldots, x_{k}$ occur in $w$ and there are no other letters.

The word $w$ can uniquely be represented in the form

$$
w=x_{1} \ldots x_{l} w^{\prime} x_{k-r+1} \ldots x_{k},
$$

where $l, r \geq 0, l+r<k$, the letters $x_{1}, \ldots, x_{l}, x_{k-r+1}, \ldots, x_{k}$ do not occur in $w^{\prime}$, the first letter of the word $w^{\prime}$ is distinct from $x_{l+1}$ or the letter $x_{l+1}$ occurs in $w^{\prime}$ more than once, and the last letter of $w^{\prime}$ is distinct from $x_{k-r}$ or the letter $x_{k-r}$ occurs in $w$ more than once. We prove the following refinement of Lemma 29.

(*) Under the above assumptions, the following identity is fulfilled in $V$ :

$$
x_{1} \ldots x_{l}\left(x_{l+1} \ldots x_{k-r}\right) x_{k-r+1} \ldots x_{k}=x_{1} \ldots x_{l}\left(x_{l+1} \ldots x_{k-r}\right)^{n+1} x_{k-r+1} \ldots x_{k} .
$$

We assume that $(*)$ is proved for $l=r=0$ and show that $(*)$ is valid in the general case. In the variety $V$, we consider the free semigroup $S$ with a countable set of generators. On $S$, we introduce the following binary relation $\sigma:(u, v) \in \sigma$ if and only if $L u R=L v R$ for all elements $L, R \in S$ such that $L$ is the product of at least $l$ elements and $R$ is the product of at least $r$ elements of $S$. Clearly, $\sigma$ is a congruence on $S$, and the identity $x_{l+1} \ldots x_{k-r}=w^{\prime}$ is fulfilled in the semigroup $S / \sigma$. By our assumption, the identity $x_{l+1} \ldots x_{k-r}=\left(x_{l+1} \ldots x_{k-r}\right)^{n+1}$ is fulfilled in $S / \sigma$. Then the identity

$$
x_{1} \ldots x_{l}\left(x_{l+1} \ldots x_{k-r}\right) x_{k-r+1} \ldots x_{k}=x_{1} \ldots x_{l}\left(x_{l+1} \ldots x_{k-r}\right)^{n+1} x_{k-r+1} \ldots x_{k}
$$

is fulfilled in the semigroup $S$, and therefore, in the variety $V$.

Thus, it remains to prove the following statement, which coincides with $(*)$ in the case where $l=r=0$.

(**) Let $V$ be a variety of semigroups of period $n$. Assume that in $V$ we have the identity $x_{1} x_{2} \ldots x_{k}=w$, where $|w|>k, c(w)=\left\{x_{1}, \ldots, x_{k}\right\}$, the first letter of the word $w$ is distinct from $x_{1}$ or the letter $x_{1}$ occurs in $w$ more than once, and the last letter of $w$ is distinct from $x_{k}$ or the letter $x_{k}$ occurs in $w$ more than once. Then the identity $x_{1} x_{2} \ldots x_{k}=\left(x_{1} x_{2} \ldots x_{k}\right)^{n+1}$ is also fulfilled in $V$.

Let $H$ denote the set of all words $w$ such that the identity $x_{1} x_{2} \ldots x_{k}=w$ is fulfilled in $V$ and the word $w$ satisfies the requirements of statement $(* *)$. Observe that $(* *)$ can easily be obtained from the following statement. 
(\#) Let $X$ be an arbitrary word with letters in $\left\{x_{1}, \ldots, x_{k}\right\}$. There is a word $u W v \in H$ and an index $i$ such that $|u|,|v| \geq k, c(W)=\left\{x_{1}, \ldots, x_{k}\right\}$, the letter $x_{i}$ occurs in the word $u W v$ at least $k+4$ times, and $X$ is a subword of $W$.

Indeed, let $X$ be the word $\left(x_{1} \ldots x_{k}\right)^{2}$. Then, in a free semigroup of $V$, the element $x_{1} \ldots x_{k}$ is divisible by $\left(x_{1} \ldots x_{k}\right)^{2}$. By Rees's theorem, $x_{1} \ldots x_{k}$ is a group element, so that $x_{1} \ldots x_{k}=\left(x_{1} \ldots x_{k}\right)^{n+1}$.

The proof of statement $(\#)$ is based on the following construction, which could be called "application of the identity $x_{1} x_{2} \ldots x_{k}=w$ to itself". Let $w=x_{i_{1}} \ldots x_{i_{N}} \in H$ $\left(1 \leq i_{1}, i_{2}, \ldots, i_{N} \leq k\right)$, and let $\pi: w=u w_{1} w_{2} \ldots w_{k} v$ be a representation of $w$ as a product with nonempty words $w_{1}, \ldots, w_{k}$ (the words $u$ and $v$ may be empty). We put $w^{\pi}=u \widetilde{W} v$, where $\widetilde{W}=w_{i_{1}} w_{i_{2}} \ldots w_{i_{N}}$. The identity $x_{1} x_{2} \ldots x_{k}=w^{\pi}$ follows from the identity $x_{1} x_{2} \ldots x_{k}=w$. It is easy to check that the word $w^{\pi}$ satisfies the requirements of statement $(* *)$, and so $w^{\pi} \in H$. It is clear that the length of the word $w^{\pi}$ is greater than that of $w$, each letter $x_{i}$ occurs in $w^{\pi}$ at least as many times as in $w$, and if all letters $x_{1}, \ldots, x_{k}$ occur in the word $w_{1} \ldots w_{k}$, then all these letters occur in the word $\widetilde{W}$. In particular, $H$ has subwords of arbitrarily large length.

For $k=1$, statement (\#) is trivial; therefore, in the sequel we assume that $k \geq 2$. We prove (\#) by induction on the length of the word $X$. Let $|X|=0$, i.e., $X$ is the empty word. In the set $H$, there is a word $w$ the length of which is at least $(k+4) k$. Then at least one letter $x_{i}$ among the $k$ letters $x_{1}, \ldots, x_{k}$ occurs in $w$ at least $k+4$ times. If the word $w$ begins with $x_{s}, s \neq 1$, then there is a representation $\pi: w=w_{1} \ldots w_{s} \ldots w_{k}$ with nonempty $w_{j}$ such that $x_{i}$ is the first letter of $w_{s}$. Then $x_{i}$ is the first letter of $w^{\pi} \in H$. Therefore, we may assume that the first letter of $w$ is either $x_{1}$ or $x_{i}$. Similarly, the last letter of the word $w$ is either $x_{k}$ or $x_{i}$. Now, let $\pi: w=w_{1} \ldots w_{i} \ldots w_{k}$ be a representation such that $\left|w_{1}\right|,\left|w_{i}\right|,\left|w_{k}\right| \geq k$. Then $w^{\pi}=w_{i_{1}} w_{i_{2}} \ldots w_{i_{N}}=u W v$, where $u=w_{1}$ or $u=w_{i}, v=w_{k}$ or $v=w_{i}$, and the word $W=w_{i_{2}} \ldots w_{i_{N-1}}$ contains all letters $x_{1}, \ldots, x_{k}$, because the letter $x_{i}$, the letter $x_{1}$ for $i_{1}=1$, and the letter $x_{k}$ for $i_{k}=k$ occur in $w$ more than once and, therefore, the two words $u$ and $v$ occur among the words $w_{i_{2}}, \ldots, w_{i_{N-1}}$.

Now, let $X=x_{j} X^{\prime}$, and assume that the word $w=u^{\prime} W^{\prime} v^{\prime} \in H$ is such that $\left|u^{\prime}\right|,\left|v^{\prime}\right| \geq$ $k, c\left(W^{\prime}\right)=\left\{x_{1}, \ldots, x_{k}\right\}$, the letter $x_{i}$ occurs in $u^{\prime} W^{\prime} v^{\prime}$ at least $k+4$ times, and $X^{\prime}$ is a subword of $W^{\prime}$. There is a representation $\pi: w=w_{1} \ldots w_{k}$ such that $W^{\prime}$ is a subword of $P=w_{i}$, so that $X^{\prime}$ is a subword of $P=w_{i}$ containing all letters $x_{1}, \ldots, x_{k}$. Since the letter $x_{i}$ occurs in $w$ at least $k+4$ times, the word $w^{\pi}$ has the form

$$
w^{\pi}=Q_{0} P Q_{1} P Q_{2} \ldots Q_{k} P Q_{k+1} P Q_{k+2}, \quad \text { where } Q_{2}=Q^{\prime} P Q^{\prime \prime} P Q^{\prime \prime \prime}
$$

(the words $Q_{0}, Q_{1}, Q_{3}, \ldots, Q_{k+2}, Q^{\prime}, Q^{\prime \prime}, Q^{\prime \prime \prime}$ may be empty). The letters $x_{k}$ and $x_{1}$ occur in the word $P$. Therefore, the word $Q_{2}=Q^{\prime} P Q^{\prime \prime} P Q^{\prime \prime \prime}$ has a two-letter subword $x_{s} x_{t}$ with $s>t$. By assumption, there exist words $Y, Z, Y_{1}$, and $Z_{1}$ such that $P=Y X^{\prime} Z=$ $Y_{1} x_{j} Z_{1}$. We put

$$
\begin{aligned}
& w_{t}=X^{\prime} Z, \quad w_{s}=Q_{s} Y_{1} x_{j}, \quad w_{q}=Q_{q} P \text { for } q \neq t-1, t, s, s+1, \\
& w_{t-1}=Q_{t-1} P Y \quad(\text { if } t>1) ; \quad w_{s+1}=Z_{1} Q_{s+1} P(\text { if } s<k) .
\end{aligned}
$$

We obtain a representation $\sigma: w^{\pi}=u w_{1} \ldots w_{k} v$ of the word $w^{\pi}$, where $u=Q_{0} P Y$ for $t=1, u=Q_{0} P$ for $t>1, v=Z_{1} Q_{k+1} P Q_{k+2}$ for $s=k$, and $v=Q_{k+1} P Q_{k+2}$ for $s<k$. In all cases, $Q_{2}$ is a subword of the product $w_{1} \ldots w_{k}$. Therefore, $x_{s} x_{t}$ is also a subword of $w_{1} \ldots w_{k}$. Consequently, the middle part $W$ of the word $\left(w^{\pi}\right)^{\sigma}=u W v$ contains a subword $w_{s} w_{t}=Q_{s} Y_{1} x_{j} X^{\prime} Z$, which contains $X=x_{j} X^{\prime}$. The properties of the operation "application of an identity to itself" show that the letter $x_{i}$ occurs in $u W v$ at least $k+4$ times, and all letters $x_{1}, \ldots, x_{k}$ occur in the word $W$. Since the words 
$u$ and $v$ contain the word $P$, the lengths of $u$ and $v$ are at least $k$. Thus, the word $u W v \in H$ satisfies all requirements of statement (\#). Therefore, statement (\#) and, with it, statements $(* *)$ and $(*)$, along with Lemma 29, are proved completely.

Remark 5. Lemma 29 was announced by A. P. Biryukov at the second All-Union school on varieties (Barnaul, 1981), but to the best of our knowledge, its proof has not been published. For this reason, we have included our proof of the lemma in the present paper 1

Lemma 30. If the identity $x=x^{n+1}$ is fulfilled in a Rees-Sushkevich variety $V$, then $V$ is generated by its completely simple semigroups.

Proof. This follows easily from Lemma 4.

Lemma 31. Let $V$ be a variety of semigroups of period $n$.

(1) If $A_{2} \notin V$, then the identity $a^{2 n} b^{2 n}=\left(a^{2 n} b^{2 n}\right)^{n+1}$ is fulfilled in all completely 0 -semigroups in $V$.

(2) If $B_{2} \notin V \subset R S_{n}$, then, for every word $a_{1} a_{2} \ldots a_{q}$ covered by cycles (the letters $a_{i}$ may fail to be distinct), the identity $a_{1} a_{2} \ldots a_{q}=a_{1} a_{2} \ldots a_{k}^{n+1} \ldots a_{q}$ (for every $k$ from 1 to $q$ ) is fulfilled in $V$.

(3) Let $A_{l}^{*} \notin V \subset R S_{n}$ (or $\left.A_{r}^{*} \notin V\right)$ and $B_{2} \notin V$. Then the identities

$$
a b c^{2 n} a=a c^{2 n} b a, \quad a b_{1} c^{2 n} b_{2} a=a c^{2 n} b_{1} b_{2} a=a b_{1} b_{2} c^{2 n} a
$$

are fulfilled in $V$ and, for every regular word $a_{1} a_{2} \ldots a_{q}$ (where the letters $a_{i}$ need not be distinct), the identity

$$
a_{1} a_{2} \ldots a_{k} \ldots a_{q}=a_{1}^{2 n} a_{k}^{2 n} a_{1} a_{2} \ldots a_{q}=a_{1} a_{2} \ldots a_{k} \ldots a_{q} a_{k}^{2 n} a_{q}^{2 n}
$$

is fulfilled in $V$ (for every $k$ from 1 to $q$ ).

(4) Let $A_{l}^{*} \notin V \subset R S_{n}$, and let $B_{2} \in V$. Let $u=v$ be an identity in which $u$ and $v$ are regular words and the first, as well as the last, letters of $u$ and $v$ coincide. If the identity $u=v$ is fulfilled in $B_{2}$ and in each group in $V$, then $u=v$ is also fulfilled in $V$.

(5) Let $A_{l}^{*} \notin V \subset R S_{n}$, and let $B_{2} \in V$. If an identity $u=v$, where $u$ and $v$ are regular words, is fulfilled in $B_{2}$, then the identities

$$
u=x^{2 n} y^{2 n} x^{2 n} y^{2 n} u, \quad u=u z^{2 n}
$$

are fulfilled in $V$. Here, $x=u u^{\perp}, y=v v^{\perp}, z=u^{\perp} u$, and $u^{\perp}$ and $v^{\perp}$ are words in the free semigroup $F(V)$ of $V$ such that $u=u u^{\perp} u$ and $v=v v^{\perp} v$.

(6) Suppose $A_{l}^{*} \notin V \subset R S_{n}$ and $B_{2} \notin V$. Let $u$ and $v$ be regular words with the same set of variables, and let $u^{\perp}$ and $v^{\perp}$ be words in the free semigroup $F(V)$ of $V$ such that $u=u u^{\perp} u$ and $v=v v^{\perp} v$. Let $x=u u^{\perp}, y=v v^{\perp}$, and $z=u^{\perp} u$. Then the identities

$$
u=x^{2 n} y^{2 n} x^{2 n} y^{2 n} u, \quad u=u z^{2 n}
$$

are fulfilled in $V$.

Proof. Let $V$ be a variety of semigroups of period $n$.

(1) If the identity $a^{2 n} b^{2 n}=\left(a^{2 n} b^{2 n}\right)^{n+1}$ is not fulfilled in some completely 0 -simple semigroup $S \in V$, then the sandwich-matrix of $S$ must have a submatrix of the form $\left(\begin{array}{ll}\alpha & \beta \\ \gamma & \delta\end{array}\right)$ with exactly one zero entry. This means that the semigroup $A_{2}$ is a factor of $S$ (i.e., a homomorphic image of a subsemigroup of $S$ ), whence $A_{2} \in V$, which contradicts the assumptions.

\footnotetext{
${ }^{1}$ Added in proof. After the Russian original of this paper had appeared, the author has learned that a (different) proof of Lemma 29 was published by V. M. Vernikov in 2008; see [23] Proposition 2.1].
} 
(2) Let $B_{2} \notin V \subset R S_{n}$. Then every completely 0-simple semigroup $S$ in $V$ is completely simple, possibly, with zero adjoined. Therefore, for each word $a_{1} \ldots a_{q}$ covered by cycles (where the letters $a_{i}$ need not be distinct), the identity $a_{1} \ldots a_{k} \ldots a_{q}=$ $a_{1} \ldots a_{k}^{n+1} \ldots a_{q}$ is fulfilled in $S$ (for every $k$ from 1 to $q$ ). Without loss of generality, we may assume that $a_{1}=a_{q}$. Using Lemma 18, we conclude that this identity is fulfilled in $V$, because the two sides of it are regular words.

(3) Suppose $A_{l}^{*} \notin V \subset R S_{n}$ and $B_{2} \notin V$. Then every completely 0-simple semigroup $S$ in $V$ is completely simple, possibly, with zero adjoined. Lemma [15] and Propositions 12 and 21 show that the identity

$$
e q A_{l, n}^{*}: z^{2 n} x^{2 n} y^{2 n} x^{2 n} y^{2 n}=z^{2 n} y^{2 n} x^{2 n} y^{2 n}
$$

is fulfilled in $V$. However, for all prime $p$, this identity is not fulfilled in the semigroups $M_{p}$ (see [5]). Therefore, all completely simple semigroups in $V$ are orthodox and the identity $a b c^{2 n} a=a c^{2 n} b a$ is fulfilled in these semigroups. By Lemma 18, the identity $a b c^{2 n} a=a c^{2 n} b a$ is fulfilled in $V$, because the two sides of this identity are regular words. The fact that the identity $a b_{1} c^{2 n} b_{2} a=a c^{2 n} b_{1} b_{2} a=a b_{1} b_{2} c^{2 n} a$ is fulfilled is proved similarly.

Let $u=a_{1} \ldots a_{q}$ be a regular word (in which the letters $a_{i}$ need not be distinct). By the statement proved in item (2), the identity

$$
a_{1} \ldots a_{q}=a_{1}^{2 n} a_{1} \ldots a_{k}^{2 n} a_{k} \ldots a_{q}
$$

is fulfilled in $V$. Combining this with $a b_{1} c^{2 n} b_{2} a=a c^{2 n} b_{1} b_{2} a$, we get

$$
a_{1} \ldots a_{k} \ldots a_{q}=a_{1}^{2 n} a_{k}^{2 n} a_{1} \ldots a_{q} .
$$

Similarly, we can prove that the identity

$$
a_{1} \ldots a_{k} \ldots a_{q}=a_{1} \ldots a_{k} \ldots a_{q} a_{k}^{2 n} a_{q}^{2 n}
$$

is also fulfilled in $V$.

(4) Suppose $A_{l}^{*} \notin V \subset R S_{n}$ and $B_{2} \in V$, and let an identity $u=v$, where $u$ and $v$ are regular words with the same first and the same last letters, be fulfilled in $B_{2}$ and in each group in $V$. Repeating the arguments of item (3), we see that the identity eqA $A_{l, n}^{*}$ is fulfilled in $V$, and all completely simple semigroups in $V$ are orthodox. Therefore, the identity $u=v$ is fulfilled in each completely simple semigroup in $V$, because this identity is fulfilled in each group in $V$. Observe that the identity $e q A_{l, n}^{*}$ is not fulfilled in $A_{2}$, i.e., $A_{2} \notin V$. Thus, all completely 0 -simple semigroups in $V$ are orthodox. We know that, in this case,

$$
\operatorname{Var}\left(C S^{0}(V)\right)=\operatorname{Var}\left(B_{2}\right)+\operatorname{Var}(C S(V))
$$

where $C S(V)$ is the class of completely simple semigroups in $V$ (see [6]). Therefore, the identity $u=v$ is fulfilled in the class $C S^{0}(V)$. By Lemma 18, this identity is also fulfilled in $V$.

(5) Suppose $A_{l}^{*} \notin V \subset R S_{n}$ and $B_{2} \in V$, and let an identity $u=v$ with regular $u$ and $v$ be fulfilled in $B_{2}$. By definition, there are elements $u^{\perp}$ and $v^{\perp}$ in $F(V)$ such that the relations $u=u u^{\perp} u$ and $v=v v^{\perp} v$ are valid in $F(V)$. Since the semigroup $B_{2}$ belongs to $V$, the identities $u=u u^{\perp} u$ and $v=v v^{\perp} v$ are fulfilled in $B_{2}$. Therefore, the identities $u u^{\perp}=\left(u u^{\perp}\right)^{2}$ and $v v^{\perp}=\left(v v^{\perp}\right)^{2}$ are also fulfilled in $B_{2}$. Since $u=v$ in $B_{2}$, we see that the identity

$$
u=u u^{\perp} u=u u^{\perp} v=u u^{\perp} v v^{\perp} v=u u^{\perp} v v^{\perp} u=\left(u u^{\perp}\right)^{2 n}\left(v v^{\perp}\right)^{2 n} u
$$

is fulfilled in $B_{2}$ for all $n$.

The last identity is also fulfilled in each group in $V$, because, in each group belonging to $V$, the values of the words $u u^{\perp}$ and $v v^{\perp}$ are equal to the identity element. By (4), we conclude that the identity $u=\left(u u^{\perp}\right)^{2 n}\left(v v^{\perp}\right)^{2 n} u$ is fulfilled in $V$. It remains to 
observe that the last identity implies the identity $u=x^{2 n} y^{2 n} x^{2 n} y^{2 n} u$, where $x=u u^{\perp}$ and $y=v v^{\perp}$. The fact that the identity $u=u z^{2 n}$, where $z=u^{\perp} u$, is fulfilled follows from the definitions.

(6) This follows directly from statement (3) of the present lemma.

Lemma 32. $\left[x^{2}=0, x y x=0\right] \subset \operatorname{Var}\left(B_{0}\right) \subset \operatorname{Var}\left(B_{2}\right)$.

Proof. Let $u=v$ be an identity fulfilled in the semigroup $B_{0}$. By Proposition 6, the words $u$ and $v$ are congruent. Therefore, these words are nonsingular or coincide. In any case, the identity $u=v$ follows from the identities $x^{2}=0$ and $x y x=0$. It remains to observe that $B_{0}$ is a subsemigroup of $B_{2}$, whence $\operatorname{Var}\left(B_{0}\right) \subset \operatorname{Var}\left(B_{2}\right)$.

We recall that a partially ordered set $M$ is said to be well partially ordered if $M$ satisfies the minimality condition and has no infinite antichains. In other words, this means that the set of minimal elements of every nonempty subset of $M$ is nonempty and finite. The following result is well known (see, e.g., [22, III.2]).

\section{Lemma 33.}

(1) The class of well partially ordered sets is closed under finite direct products.

(2) Every infinite sequence $\left\{z_{n}\right\}$ of elements of a well partially ordered set has a (nonstrictly) increasing infinite subsequence $\left\{z_{n_{k}}\right\}$ (i.e., a subsequence such that $z_{n_{k}} \leq$ $z_{n_{k+1}}$ for $\left.n_{k}<n_{k+1}\right)$.

The following propositions concern varieties defined by permutation identities. We begin with several definitions. Every nontrivial permutation identity has the form

$$
x_{1} \ldots x_{l}\left(x_{l+1} \ldots x_{m-r}\right) x_{m-r+1} \ldots x_{m}=x_{1} \ldots x_{l}\left(x_{s} \ldots x_{t}\right) x_{m-r+1} \ldots x_{m},
$$

where $l, r \geq 0, s \neq l+1$, and $t \neq m-r$. We call the numbers $l$ and $r$, respectively, the left and the right characteristic of the permutation identity in question, and the pair $(l, r)$ the characteristic of the identity. Now, let $V$ be a variety defined by permutation identities. We denote by $l(V)$ (respectively, by $r(V))$ the smallest left (respectively, right) characteristic of the defining identities. The pair $(l(V), r(V))$ is called the characteristic of the variety. It is clear that the characteristics of all permutation identities of a variety of characteristic $(l, r)$ is not less than $(l, r)$. At the same time, the variety has an identity of characteristic $(l, r)$. Indeed, $V$ has a permutation identity $u=v$ with left characteristic $l$ and a permutation identity $u_{1}=v_{1}$ with right characteristic $r$. If the sets of these identities are disjoint, then $u u_{1}=v v_{1}$ is a permutation identity of characteristic $(l, r)$.

Proposition 34 (Pollák 14]).

(1) Every set of permutation identities has a finite basis.

(2) Every permutation identity implies all identities of the same or greater characteristic except a finite number of them.

Proposition 35. Every set of varieties defined by permutation identities contains no infinite antichains and satisfies the minimality condition.

Proof. For every variety $V$, we denote by $T(V)$ the set of all permutation identities fulfilled in $V$.

Let $T_{1} \subset T_{2} \subset \cdots$ be an increasing infinite sequence of sets of permutation identities. By Proposition 34, the union of these sets has a finite basis all elements of which are already contained in some $T_{s}$. Therefore, for $n>s$, all identities in $T_{n}$ follow from $T_{s}$. Thus, to prove the proposition, it suffices to show that if there is an antichain or a strictly decreasing chain of varieties defined by permutation identities, then there is an infinite sequence of sets of permutation identities

$$
T_{1} \subset T_{2} \subset \cdots \subset T_{s} \subset T_{s+1} \subset \cdots
$$


such that, for each $s$, in $T_{s+1}$ there is an identity that does not follow from $T_{s}$.

In the second case, this claim is trivial. Indeed, if $V_{1} \supset V_{2} \supset \cdots$ is a strictly decreasing chain of varieties defined by permutation identities, then $T\left(V_{1}\right) \subset T\left(V_{2}\right) \subset \cdots$, and, for each $s$, in $T\left(V_{s+1}\right)$ there is an identity that does not follow from $T\left(V_{s}\right)$ (otherwise $\left.T\left(V_{s+1}\right)=T\left(V_{s}\right)\right)$, whence $V_{s+1}=V_{s}$.

Now, let $\mathcal{A}$ be an infinite antichain of varieties defined by permutation identities, and let $\mathcal{A}_{1} \supset \mathcal{A}_{2} \supset \cdots$ be a decreasing sequence of infinite antichains contained in $\mathcal{A}$. For every $s$, we put $T_{s}=\bigcap_{V \in \mathcal{A}_{s}} T(V)$. By induction on $s$, we construct a decreasing sequence of antichains such that, for each $s$ in $T_{s+1}$, there is an identity that does not follow from $T_{s}$.

We put $\mathcal{A}_{1}=\mathcal{A}$. Let $s \geq 1$; suppose that antichains $\mathcal{A}_{1}, \ldots, \mathcal{A}_{s}$ with the required property have already been constructed. Since every set of pairs of positive integers has only finitely many minimal elements, there is a variety $U \in \mathcal{A}_{s}$ and an infinite subset $\mathcal{A}_{s}^{\prime} \not \ngtr U$ of $\mathcal{A}_{s}$ such that $\left(l_{U}, r_{U}\right) \leq\left(l_{V}, r_{V}\right)$ for all $V \in \mathcal{A}_{s}^{\prime}$. Among the identities of the variety $U$, there is an identity $q$ with characteristic equal to $\left(l_{U}, r_{U}\right)$. By Proposition 34, there is a finite set (possibly, empty) of identities $q_{1}, \ldots, q_{n}$ that do not follow from $q$ and $T_{s}$ and such that every identity of characteristic at least $\left(l_{U}, r_{U}\right)$ follows from $q$ and $T_{s}$ or coincides with one of the identities $q_{1}, \ldots, q_{n}$. If each identity $q_{i}$ is contained in $T(V)$ only for a finite number of varieties $V \in \mathcal{A}_{s}^{\prime}$, then there is a variety $W \in \mathcal{A}_{s}^{\prime}$ such that none of the identities $q_{i}$ is an identity in $W$. Then all identities of the variety $W$ follow from $T_{s} \subset T(U)$ and $q \in T(U)$, and therefore, these identities belong to $T(U)$. Consequently, the variety $U$ is contained in $W$, which contradicts the fact that $U$ and $W$ are different elements of an antichain. Thus, for some $i$, the set of varieties in $\mathcal{A}_{s}^{\prime}$ for which $q_{i}$ is an identity is finite. We take this set as $\mathcal{A}_{s+1}$. An identity $q_{i}$ that does not follow from $T_{s}$ belongs to $T_{s+1}=\bigcap_{V \in \mathcal{A}_{s+1}} T(V)$. Therefore, the antichain $\mathcal{A}_{s+1}$ has the required property.

Proposition 36. The lattice of subvarieties of the variety $\left[x^{2}=0, x y x=0\right]$ satisfies the minimality condition and has no infinite antichains.

Proof. This follows from Proposition 35 and Lemma 33. Indeed, it suffices to observe that every variety $V \subset\left[x^{2}=0, x y x=0\right]$ is uniquely determined by the pair $\left(E q N(V), n_{D}(V)\right)$, where $E q N(V)$ is the set of permutation identities fulfilled in the variety $V$.

Proposition 37. For the Rees-Sushkevich varieties $V \subset R S_{n}$, the following properties are valid:

(1) $A_{2} \notin V \Leftrightarrow$ the identity $x^{2 n} y x^{2 n}=x^{2 n} y^{n+1} x^{2 n}$ is fulfilled in $V$;

(2) $B_{2} \notin V \Leftrightarrow$ the identity $x y x=x y^{n+1} x$ is fulfilled in $V$;

(3) $A_{0} \notin V \Leftrightarrow$ the identity $\left(x^{2 n} y^{2 n}\right)^{n+1}=x^{2 n} y^{2 n}$ is fulfilled in $V$;

(4) $B_{0} \notin V \Leftrightarrow$ the identity $x^{2 n} y z^{2 n}=x^{2 n} y^{n+1} z^{2 n}$ is fulfilled in $V$;

(5) $M_{p} \notin V$ for all $p \Leftrightarrow$ the identity $\left(x^{2 n} y^{2 n}\right)^{n+1}=\left(x^{2 n} y^{2 n}\right)^{n+2}$ is fulfilled in $V$.

Proof. It is easily seen that the identities mentioned above are not fulfilled in the corresponding semigroups $A_{2}, B_{2}, A_{0}, B_{0}$, and $M_{p}$. Therefore, the implications $\Leftarrow$ are valid. We prove the reverse implications.

(1) Let $A_{2} \notin V \subset R S_{n}$. Then all completely 0-simple semigroups in $V$ are Rees matrix semigroups with a block-diagonal sandwich-matrix over a group of exponent dividing $n$. It is easy to check that the identity $x^{2 n} y x^{2 n}=x^{2 n} y^{n+1} x^{2 n}$ is fulfilled in such semigroups. Lemma 4 on regular elements implies that every identity $u=v$ in which $u$ and $v$ are regular words is fulfilled in $V$ if $u=v$ is fulfilled in $V^{\prime}$. Therefore, the identity $x^{2 n} y x^{2 n}=$ $x^{2 n} y^{n+1} x^{2 n}$ is fulfilled in $V$. 
(2) Let $B_{2} \notin V \subset R S_{n}$. Then all completely 0-simple semigroups in $V$ are completely simple, possibly, with zero adjoined. It is easy to check that the identity $x y x=x y^{n+1} x$ is fulfilled in such semigroups. Next, repeating the above argument, we see that the identity $x y x=x y^{n+1} x$ is fulfilled in $V$.

(3) Let $A_{0} \notin V$. By Proposition 7 , all semigroups in $V$ are regularly closed. This means that $x^{2 n} y^{2 n}$ is a regular element of the free semigroup of the variety $V \subset R S_{n}$. Hence, the relation $\left(x^{2 n} y^{2 n}\right)^{n+1}=x^{2 n} y^{2 n}$ is fulfilled in this free semigroup. However, every relation among elements of the free semigroup of a variety is an identity of this variety.

(4) Let $B_{0} \notin V$. We assume that the identity $x^{2 n} y z^{2 n}=x^{2 n} y^{n+1} z^{2 n}$ is not fulfilled in a semigroup $S \in V$. By Proposition [6, in all semigroups in $V$ the set of regular elements is a biideal. Therefore, $x^{2 n} y z^{2 n}$ and $x^{2 n} y^{n+1} z^{2 n}$ are regular elements of the semigroup $S$. Lemma 4 on regular elements shows that the identity $x^{2 n} y z^{2 n}=x^{2 n} y^{n+1} z^{2 n}$ is not fulfilled in some completely 0 -simple semigroup $\bar{S} \in V$. Since $B_{0} \notin V$, we have $B_{2} \notin V$. Thus, all completely 0 -simple semigroups in $V$ are completely simple, possibly, with zero adjoined. It is easy to check that the identity $y=y^{n+1}$ is fulfilled in such semigroups. Therefore, the identity $x^{2 n} y z^{2 n}=x^{2 n} y^{n+1} z^{2 n}$ is also fulfilled, a contradiction.

(5) Let $M_{p} \notin V$ for all $p$. This means that all completely simple semigroups in $V$ are orthodox. Therefore, the identity $x^{2 n} y^{2 n}=\left(x^{2 n} y^{2 n}\right)^{2}$ is fulfilled in such semigroups. Thus, the identity

$$
x^{2 n} y^{2 n}\left(x^{2 n} y^{2 n}\right)^{n}=\left(x^{2 n} y^{2 n}\right)^{2}\left(x^{2 n} y^{2 n}\right)^{n}
$$

is also fulfilled. If the latter identity is not fulfilled in $V$, then, by Lemma 4 on regular elements, this identity is not fulfilled in some completely 0 -simple semigroup $S$ in $V$ for some elements $x$ and $y$. Then, in this semigroup, we have $\left(x^{2 n} y^{2 n}\right)^{2} \neq 0$. This means that the element $x^{2 n} y^{2 n}$ belongs to a subgroup of the semigroup $S$, which implies that $x^{2 n} y^{2 n}=\left(x^{2 n} y^{2 n}\right)^{n+1}$, because all subgroups $S \in V \subset R S_{n}$ are $n$-periodic. This implies that $(*)$ is fulfilled in $S$, a contradiction.

Lemma 38. Let $V \subset R S_{n}$ be a Rees-Sushkevich variety. If $B_{0} \notin V$, then the identities

(*) $\quad x^{2 n} z_{1} z_{2} y^{2 n}=x^{2 n} z_{1}^{n+1} z_{2} y^{2 n}=x^{2 n} z_{1} z_{2}^{n+1} y^{2 n}$,

(**) $u=u^{n+1}$ ( $u$ is a regular word)

are fulfilled in $V$.

Proof. Let $V$ be a Rees-Sushkevich variety included in $R S_{n}$. Let $B_{0} \notin V$. Then $B_{2} \notin V$, and all completely 0 -simple semigroups in $V$ are completely simple, possibly, with zero adjoined. This means that, in such semigroups, we have $x=x^{n+1}$, so that $(*)$ and $(* *)$ are fulfilled. By Lemma 18, $(* *)$ is fulfilled in $V$. We assume that the identity $x^{2 n} z_{2} z_{2} y^{2 n}=x^{2 n} z_{1}^{n+1} z_{2} y^{2 n}$ is not fulfilled in $V$, i.e., it is not fulfilled in a semigroup $S$ in $V$. By Proposition [6, the elements $x^{2 n} z_{2} z_{2} y^{2 n}$ and $x^{2 n} z_{1}^{n+1} z_{2} y^{2 n}$ are regular in $S$. If these elements are distinct, then, by Lemma 4 on regular elements, they can be separated by a homomorphism into a completely 0 -simple semigroup. However, it was proved above that identity $(*)$ is fulfilled in completely 0 -simple semigroups in $V$, a contradiction.

Lemma 39. Let $V$ be a Rees-Sushkevich variety of period $n$. If $V$ is generated by a completely 0-simple semigroup and a finite semigroup and $B_{0} \notin V$, then the identities

(a) $x_{1} x_{2} \ldots x_{k} z^{2 n}=x_{1} x_{2} \ldots x_{k}^{n+1} z^{2 n}$,

(b) $z^{2 n} x_{1} x_{2} \ldots x_{k}=z^{2 n} x_{1}^{n+1} x_{2} \ldots x_{k}$

are fulfilled in $V$ for some positive integer $k$.

Proof. Let $V$ be a variety belonging to $R S_{n}$, and let $B_{0} \notin V$. Then $B_{2} \notin V$, and all completely 0 -simple semigroups in $V$ are completely simple, possibly, with zero adjoined. This means that the identity $x=x^{n+1}$ is fulfilled in these semigroups. 
Let $V=\operatorname{Var}(S)+\operatorname{Var}\left(C S^{0} V\right)$, where $S$ is a semigroup of finite order $m$. Then, for all $x_{1}, x_{2}, \ldots, x_{m}, x_{m+1}$ in $S$, for some $k \leq m$, and for an idempotent $g$ in $S$, we have $x_{1} x_{2} \ldots x_{k} x_{k+1} \ldots x_{m+1}=x_{1} x_{2} \ldots x_{k} g x_{k+1} \ldots x_{m+1}$. Indeed, by the pigeonhole principle, the sequence

$$
x_{1}, x_{1} x_{2}, \ldots, x_{1} x_{2} \ldots x_{m+1}
$$

has equal elements $x_{1} x_{2} \ldots x_{k}$ and $x_{1} x_{2} \ldots x_{k} x_{k+1} \ldots x_{l+1}(k \leq l \leq m)$. Then, for each $q$, we have

$$
x_{1} x_{2} \ldots x_{k}=x_{1} x_{2} \ldots x_{k}\left(x_{k+1} \ldots x_{l+1}\right)^{q} .
$$

In a finite semigroup, some power of an arbitrary element is idempotent. Therefore, $x_{1} x_{2} \ldots x_{k}=x_{1} x_{2} \ldots x_{k} g$ for an idempotent $g$. Using Lemma 38, we obtain

$$
\begin{gathered}
x_{1} x_{2} \ldots x_{k} x_{k+1} \ldots x_{m+1} z^{2 n}=x_{1} x_{2} \ldots x_{k} g x_{k+1} \ldots x_{m+1} z^{2 n} \\
\quad=x_{1} x_{2} \ldots x_{k} g x_{k+1} \ldots x_{m+1}^{n+1} z^{2 n}=x_{1} x_{2} \ldots x_{m+1}^{n+1} z^{2 n} .
\end{gathered}
$$

The fact that the identity $z^{2 n} x_{1} x_{2} \ldots x_{m+1}=z^{2 n} x_{1}^{n+1} x_{2} \ldots x_{m+1}$ is fulfilled in $S$ is proved similarly. It remains to observe that these identities are fulfilled in the variety $\operatorname{Var}\left(C S^{0} V\right)$.

Proposition 40. Let $V$ be a Rees-Sushkevich variety of period $n$. If $V$ is generated by a completely 0-simple semigroup and a finite semigroup and $B_{0} \notin V$, then $V$ is a diverse variety.

Proof. Let $V$ be a variety included in $R S_{n}$, and let $B_{0} \notin V$. Then $B_{2} \notin V$, and all completely 0-simple semigroups in $V$ are completely simple, possibly, with zero adjoined. This means that, in these semigroups, we have $x=x^{n+1}$. By Lemma 39, the identities

(a) $x_{1} x_{2} \ldots x_{k} z^{2 n}=x_{1} x_{2} \ldots x_{k}^{n+1} z^{2 n}$,

(b) $z^{2 n} x_{1} x_{2} \ldots x_{k}=z^{2 n} x_{1}^{n+1} x_{2} \ldots x_{k}$

are fulfilled in $V$ for some $k$.

Now, let $V=\operatorname{Var}\left(C S^{0} V\right)+\operatorname{Var}(S)$, where $S$ is a finite semigroup of order $m$. If $x_{1}, \ldots, x_{m}, x_{m+1}$ are arbitrary elements of $S$, then we have $x_{1} \ldots x_{k_{1}} x_{k_{1}+1} \ldots x_{m+1}=$ $x_{1} \ldots x_{k_{1}} g_{x} x_{k_{1}+1} \ldots x_{m+1}$ for some $k_{1} \leq m$ and an idempotent $g_{x}$. Similarly, for all $y_{1}, \ldots, y_{m}, y_{m+1}$ in $S$, some $k_{2} \leq m$, and some idempotent $g_{x}$, we have

$$
y_{1} \ldots y_{k_{1}} y_{k_{1}+1} \ldots y_{m+1}=y_{1} \ldots y_{k_{1}} g_{y} y_{k_{1}+1} \ldots y_{m+1} \text {. }
$$

Using identity (a), we see that, for all $z \in S$,

$$
\begin{aligned}
& x_{1} x_{2} \ldots x_{k} x_{k+1} \ldots x_{m+1} z y_{1} y_{2} \ldots y_{k} y_{k+1} \ldots y_{m+1} \\
& \quad=x_{1} x_{2} \ldots x_{k} g_{x} x_{k+1} \ldots x_{m+1} z y_{1} y_{2} \ldots y_{k} g_{y} y_{k+1} \ldots y_{m+1} \\
& \quad=x_{1} x_{2} \ldots x_{k_{1}} g_{x} x_{k_{1}+1} \ldots x_{m+1} z^{n+1} y_{1} y_{2} \ldots y_{k_{2}} g_{y} y_{k_{2}+1} \ldots y_{m+1} \\
& \quad=x_{1} x_{2} \ldots x_{k} x_{k+1} \ldots x_{m+1} z^{n+1} y_{1} y_{2} \ldots y_{k} y_{k+1} \ldots y_{m+1} .
\end{aligned}
$$

This means that the identity

$$
x_{1} x_{2} \ldots x_{m+1} z y_{1} y_{2} \ldots y_{m+1}=x_{1} x_{2} \ldots x_{m+1} z^{n+1} y_{1} y_{2} \ldots y_{m+1}
$$

is fulfilled in $S$ (and, therefore, also in $\operatorname{Var}(S)$ ). It remains to observe that this identity is also fulfilled in the variety $\operatorname{Var}\left(C S^{0} V\right)$.

Proposition 41. Let $T$ be an arbitrary class of semigroups belonging to $R S_{n}$, and let $T^{\#}$ be the class of completely 0-simple principal factors of the semigroups in $T$. Then $(\operatorname{Var}(T))^{\prime}=\operatorname{Var}\left(T^{\#}\right)$. 
Proof. It is clear that $\operatorname{Var}\left(T^{\#}\right) \subset(\operatorname{Var}(T))^{\prime}$. We prove the reverse inclusion $(\operatorname{Var}(T))^{\prime} \subset$ $\operatorname{Var}\left(T^{\#}\right)$. Assuming the contrary, we find an identity $u=v$ that is fulfilled in $\operatorname{Var}\left(T^{\#}\right)$ but not in $(\operatorname{Var}(T))^{\prime}$. This means that the identity $u=v$ is not fulfilled in some completely 0 -simple semigroup $K$ in $\operatorname{Var}(T)$. By Lemma 27 the identity $u^{f}=v^{f}$ obtained from $u=v$ by replacing each letter $x$ by the word $x y_{x} t z_{x} x$, where $t, y_{x}$, and $z_{x}$ are letters that do not occur in $u$ and $v$, is not fulfilled in $K$, and consequently, nor in $(\operatorname{Var}(T))^{\prime}$. Then the identity $u^{f}=v^{f}$ is not fulfilled in $\operatorname{Var}(T)$, i.e., in some semigroup $S \in T$. The words $u^{f}$ and $v^{f}$ are regular, and the values of regular words are regular elements in the Rees-Sushkevich semigroups (see [9]). Therefore, by Lemma 4 on regular elements, the identity $u^{f}=v^{f}$ is not fulfilled in a completely 0-simple principal factor of the semigroup $S$, and therefore, also in the class $T^{\#}$. Since the identity $u^{f}=v^{f}$ follows from $u=v$, the identity $u=v$ is not fulfilled in the class $T^{\#}$, which contradicts the assumption.

Proposition 42. Let $V$ be a Rees-Sushkevich variety. An identity $u=v$ is fulfilled in $V^{\prime}$ if and only if the identity $u^{f}=v^{f}$ obtained from $u=v$ by replacing each letter $x$ by the word $x y_{x} t z_{x} x$, where $t, y_{x}$, and $z_{x}$ are letters that do not occur in the words $u$ and $v$, is fulfilled in $V$.

Proof. If $u=v$ is fulfilled in $V^{\prime}$, then its consequence $u^{f}=v^{f}$ is also fulfilled in $V^{\prime}$. By the lemma on regular elements, the identity $u^{f}=v^{f}$ is fulfilled in $V$. Conversely, let the identity $u^{f}=v^{f}$ be fulfilled in $V$. Then this identity is fulfilled in $V^{\prime} \subset V$, and, by Lemma 27, the identity $u=v$ is fulfilled in $V^{\prime}$.

Lemma 43. Let $V$ be a Rees-Sushkevich variety of period $n$. If the identity eq $_{1}$ is fulfilled in $V$, then the identity $e q_{1 n}$ is also fulfilled in $V$. If the identity $e q_{2}$ is fulfilled in $V$, then the identity $e q_{2 n}$ is also fulfilled in $V$.

Proof. This follows directly from Propositions [37, 7, and 6.

\section{$\S 6$. Proofs of MAin Results}

Proof of Theorem 11. Let $V \subset R S_{n}$ be a Rees-Sushkevich variety. We must prove that $V$ is generated by its 0 -simple semigroups and the set of finite semigroups $\mathfrak{A}_{V} \cup \mathfrak{N}_{V}$ defined in Subsection 2.16. Let $F(V)$ be the free semigroup in $V$ over a countable alphabet. To prove the theorem, we must show that if an identity $u=v$ is not fulfilled in $V$, then the words $u$ and $v$ can be separated by a homomorphism $f: F(V) \rightarrow S$, where $S \in V$ and either $S$ is a completely 0-simple semigroup in $V$, or $S \in \mathfrak{A}_{V} \cup \mathfrak{N}_{V}$. We consider all possible cases.

1) $Y_{2} \notin V$. Then the identity $u^{\#}=v^{\#}$ such that $c\left(u^{\#}\right) \neq c\left(v^{\#}\right)$ is fulfilled in $V$. This identity implies an identity of the form $x^{2 n}=P y Q$, where $x$ and $y$ are variables and $P$ and $Q$ are words. Since the identity $\left(\mathrm{II}_{n}\right)$ is fulfilled in $V$, we see that, in the semigroup $F(V)$, every nonsingular word $w$ is divisible by an arbitrary element of $F(V)$, and therefore, $w$ belongs to the kernel $\operatorname{ker} F(V)$ of $F(V)$ (we recall that the kernel of a semigroup $S$ is the intersection of all ideals of $S$ ). The kernel of a semigroup is a simple semigroup. By the Rees theorem, a simple periodic semigroup is completely simple (see [7]). Therefore, every nonsingular word is regular.

1.1. $u$ and $v$ are nonsingular words. Then $u$ and $v$ are regular elements of the semigroup $F(V)$. By the lemma on regular elements, the words $u$ and $v$ can be separated by an epimorphism to a completely 0 -simple semigroup in $V$.

1.2. One of the words $u$ or $v$ is singular and the other is nonsingular. For definiteness, let $v$ be nonsingular. Then $v$ is a regular element of $F(V)$. Let $k=|u|$. If $C N_{k} \in V$, then the words $u$ and $v$ can be separated by a homomorphism $f: F(V) \rightarrow C N_{k}$, because the identity $u=v$ is not fulfilled in $C N_{k}$. Let $C N_{k} \notin V$. Then, by Lemma 3, there is a 
nonsingular word $w$ such that the identity $x_{1} x_{2} \ldots x_{k}=w$ is fulfilled in $V$. Consequently, $u$ is also a regular element of $F(V)$. By the lemma on regular elements, we can separate $u$ and $v$ by an epimorphism to a completely 0 -simple semigroup in $V$.

1.3. $u$ and $v$ are singular words.

1.3.1. $c(u) \neq c(v)$. Let $k=\min (|u|,|v|)$. If $C N_{k} \in V$, then the words $u$ and $v$ can be separated by a homomorphism $f: F(V) \rightarrow C N_{k} \in V$, because the identity $u=v$ is not fulfilled in the semigroup $C N_{k}$. If $C N_{k} \notin V$, then, by Lemma 3 the identity $x_{1} x_{2} \ldots x_{k}=w$ is fulfilled in $V$ for some nonsingular word $w$. Then $u$ and $v$, along with $w$, are regular elements of the semigroup $F(V)$. By the lemma on regular elements, $u$ and $v$ can be separated by an epimorphism to a completely 0 -simple semigroup in $V$.

1.3.2. $c(u)=c(v)$. Consequently, $u=v$ is a permutation identity. Let $|u|=k$. If $k \geq$ $n_{D}(V)$, then the identity $x_{1} x_{2} \ldots x_{k}=w$ is fulfilled in $V$ for some nonsingular word $w$. Therefore, $u$ and $v$, along with $w$, are regular elements of the semigroup $F(V)$. By the lemma on regular elements, the elements $u$ and $v$ can be separated by an epimorphism to a completely 0-simple semigroup in $V$. If $k<n_{D}(V)$ and $N_{k} \in V$, then the words $u$ and $v$ can be separated by a homomorphism $f: F(V) \rightarrow N_{k}$, because the identity $u=v$ is not fulfilled in $N_{k}$.

It remains to consider the case where $k<n_{D}(V)$ and $N_{k} \notin V$. Since $N_{k} \notin V$, Lemma 2 implies that the identity $x_{1} x_{2} \ldots x_{k}=w$ is fulfilled in $V$ for a word $w$ distinct from $x_{1} x_{2} \ldots x_{k}$. If the word $w$ is not a permutation of the word $x_{1} x_{2} \ldots x_{k}$, then $w, u$, and $v$ are regular elements of the semigroup $F(V)$. By the lemma on regular elements, the words $u$ and $v$ can be separated by an epimorphism to a completely 0 -simple semigroup in $V$. Now, let $w$ be a permutation of the word $x_{1} x_{2} \ldots x_{k}$. Then a permutation identity of length less than $n_{D}(V)$ is fulfilled in $V$. Consequently, the radical $\mathfrak{R}(V)$ is distinct from 0. By Lemma 25, we have $\mathfrak{R}(V)=V \cap\left[x^{2}=0, x y x=0\right]$.

Since the words $x^{2}$ and $x y x$ are nonsingular, they belong to $\operatorname{ker} F(V)$. Consequently, the relations $x^{2}=0$ and $x y x=0$ are fulfilled in the Rees factor $F(V) / \operatorname{ker} F(V) \in V$. Thus, $\operatorname{ker} F(V) \in V \cap\left[x^{2}=0, x y x=0\right]=\mathfrak{R}(V)$. Let $S$ be a subsemigroup of $F(V) / \operatorname{ker} F(V)$ generated by the elements of $c(u)$. Since $S$ is finitely generated, it is a finite semigroup in $\mathfrak{R}(V)$. Consequently, $S \in \mathfrak{N}_{V}$. Since $k<n_{D}(V)$, the words $u$ and $v$ do not belong to $\operatorname{ker} F(V)$, so that $u$ and $v$ are distinct elements of $S$. Thus, the words $u$ and $v$ can be separated by the canonical epimorphism $f: F(V) \rightarrow S$ to a semigroup $S \in \mathfrak{N}_{V}$.

2) $Y_{2} \in V$. We note that $Y_{2}$ is a completely 0-simple semigroup. If $c(u) \neq c(v)$, then the words $u$ and $v$ can be separated by a homomorphism $f: F(V) \rightarrow Y_{2}$. Therefore, we may assume that $c(u)=c(v)$.

2.1. $B_{0} \notin V$. We denote by $K(V)$ the subsemigroup of $F(V)$ generated by nonsingular words. It is clear that $K(V)$ is an ideal in $F(V)$.

2.1.1. $u \notin K(V)$ (or $v \notin K(V)$ ). Let $|u|=k$. If $k \geq n_{D}(V)$, then the identity $x_{1} x_{2} \ldots x_{k}=w$ is fulfilled in $V$ for a nonsingular word $w$. Consequently, $u \in K(V)$, which contradicts the assumption. If $k<n_{D}(V)$ and $N_{k} \in V$, then the words $u$ and $v$ can be separated by a homomorphism $f: F(V) \rightarrow N_{k}$, because the identity $u=v$ is not fulfilled in $N_{k}$.

It remains to consider the case where $k<n_{D}(V)$ and $N_{k} \notin V$. Since $N_{k} \notin V$, Lemma 2 implies that there is a word $w$ distinct from $x_{1} x_{2} \ldots x_{k}$ such that the identity $x_{1} x_{2} \ldots x_{k}=w$ is fulfilled in $V$. If $w$ is not a permutation of the word $x_{1} x_{2} \ldots x_{k}$, then $u \in K(V)$, which contradicts the assumption. Consequently, $w$ is a permutation of the word $x_{1} x_{2} \ldots x_{k}$. Thus, a permutation identity of length less than $n_{D}(V)$ is fulfilled in $V$, and the radical $\mathfrak{R}(V)$ is nonzero. 
We consider the subsemigroup $S$ of $F(V) / K(V)$ generated by the elements of $c(u)$. The words $x^{2}$ and $x y x$ are nonsingular. Consequently, $x^{2}$ and $x y x$ belong to $K(V)$, and, hence, to $F(V) / K(V)$. Therefore, the relations $x^{2}=0$ and $x y x=0$ are also fulfilled in $S$. Thus, $S$ is finitely generated; therefore, it is a finite semigroup in $V \cap\left[x^{2}=0, x y x=\right.$ $0]=\mathfrak{R}(V)$. Thus, $S \in \mathfrak{N}_{V}$. Since $u \notin K(V)$ and $k<n_{D}(V)$, the words $u$ and $v$ are distinct in $S$ and can be separated by the canonical epimorphism $f: F(V) \rightarrow S \in \mathfrak{N}_{V}$.

2.1.2. $u, v \in K(V)$. Without loss of generality, we may assume that the words $u$ and $v$ are nonsingular. Let $u \equiv w_{1} u_{1} w_{2} u_{2} \ldots w_{m} u_{m} w_{m+1}$ be the canonical decomposition of the 1 st kind of the word $u$, where the $u_{i}$ are regular words and the $w_{i}$ are singular words (or empty symbols), and different words have no letters in common. By Proposition 6 , the word $u_{1} w_{2} u_{2} \ldots w_{m} u_{m}$ is a regular element of the free semigroup $F(V)$ in $V$. Thus, the element $u$ can be represented in the form $u=w_{u} u^{\prime} w_{u}^{*}$, where the $w_{u}$ and $w_{u}^{*}$ are singular words or empty symbols, and $u^{\prime}$ is a regular element of the semigroup $F(V)$. Similarly, we have $v=w_{v} v^{\prime} w_{v}^{*}$ for a regular element $v^{\prime}$ and singular or empty words $w_{v}$ and $w_{v}^{*}$ that have no common letters. The representations $u=w_{u} u^{\prime} w_{u}^{*}$ and $v=w_{v} v^{\prime} w_{v}^{*}$ with regular $u^{\prime}$ and $v^{\prime}$ and singular $w_{u}, w_{v}, w_{u}^{*}$, and $w_{v}^{*}$ are not unique. We choose them so that the sum $\left|w_{u}\right|+\left|w_{u}^{*}\right|+\left|w_{v}\right|+\left|w_{v}^{*}\right|$ of their lengths be minimal possible.

Let $k=\left|w_{u}\right|$ and $q=\left|w_{u}^{*}\right|$. Let $k \geq 1$. Since in the case in question we have $B_{0} \notin V$, so that $A_{0} \notin V$, Proposition 37] shows that the identity $x^{2 n} y^{2 n}=\left(x^{2 n} y^{2 n}\right)^{n+1}$ is fulfilled in the variety $V$. It follows that the identity

$$
q_{l, k}^{*}: x_{1} x_{2} \ldots x_{k} z^{2 n}=x_{1} x_{2} \ldots x_{k}^{n+1} z^{2 n}
$$

is not fulfilled in $V$, because otherwise the identity

$$
x_{1} x_{2} \ldots x_{k} z^{2 n}=x_{1} x_{2} \ldots x_{k}^{n+1} z^{2 n}=\left(x_{1} x_{2} \ldots x_{k-1}\right)\left(x_{k}\left(x_{k}^{2 n} z^{2 n}\right)^{n+1}\right)
$$

is fulfilled in $V$, which contradicts the minimality of $\left|w_{u}\right|+\left|w_{u}^{*}\right|+\left|w_{v}\right|+\left|w_{v}^{*}\right|$. If, moreover, $S L_{k} \notin V$, then Proposition 23 implies that the identity

$$
q_{l, k}: x_{1} x_{2} \ldots x_{k} x^{2 n} y^{2 n} x^{2 n} y^{2 n}=x_{1} x_{2} \ldots x_{k} y^{2 n} x^{2 n} y^{2 n}
$$

is fulfilled in $V$ because $q_{l, k}^{*}$ is not fulfilled in $V$.

A similar claim is valid if $k=0$ and $L_{2} \notin V$. Then all completely 0 -simple semigroups in $V$ are orthodox and completely simple, possibly, with zero adjoined, and the identity $q_{l, 0}$ is fulfilled in these semigroups. By Lemma 18, the identity $q_{l, 0}$ is fulfilled in $V$. The "right" analogs of these statements are also valid: if $q \geq 1$, then the identity $q_{r, q}^{*}$ is not fulfilled in $V$, and if, moreover, $S R_{q} \notin V$ or $q=0$ and $R_{2} \notin V$, then the identity $q_{r, q}$ is fulfilled in $V$.

Now, we split the argument into several steps.

a) $w_{u} \not \equiv w_{v}$ or $w_{u}^{*} \not \equiv w_{v}^{*}$. For definiteness, let $w_{u} \not \equiv w_{v}$, and let $k=\left|w_{u}\right|>0$ (the different words $w_{u}$ and $w_{v}$ cannot be empty simultaneously). Since the identity $q_{l, k}^{*}$ is not fulfilled in $V$, Proposition 24 implies that $S L_{k}^{*} \in V$. Since the words $w_{u}$ and $w_{v}$ are distinct, Lemma 17 shows that the identity $u=v$ is not fulfilled in $S L_{k}^{*}$. Therefore, the words $u$ and $v$ can be separated by the canonical homomorphism of $F(V)$ to the semigroup $S L_{k}^{*} \in V$.

b) $w_{u} \equiv w_{v}, S L_{k} \in V$ for $k>0$, or $L_{2} \in V$ for $k=0$, and the first letters of the words $u^{\prime}$ and $v^{\prime}$ are distinct. For $k>0$, the identity $u=v$ is not fulfilled in $S L_{k} \in V$ by Lemma 16. Therefore, the words $u$ and $v$ can be separated by the canonical epimorphism of the semigroup $F(V)$ onto the semigroup $S L_{k} \in \mathfrak{A}_{V}$ (respectively, to the completely 0 -simple semigroup $L_{2} \in V$ ). By duality, we can consider the case where

c) $w_{u}^{*} \equiv w_{v}^{*}, S R_{q} \in V$ for $q>0$, or $R_{2} \in V$ for $q=0$, and the last letters of the words $u^{\prime}$ and $v^{\prime}$ are distinct. 
d) $w_{u} \equiv w_{v}, w_{u}^{*} \equiv w_{v}^{*}, S L_{k} \notin V$ for $k>0$, or $L_{2} \notin V$ for $k=0$, and $S R_{q} \notin V$ for $q>0$, or $R_{2} \notin V$ for $q=0$. Then $c\left(u^{\prime}\right)=c\left(v^{\prime}\right)$ because $c(u)=c(v)$, and, as has been proved, the identity

$$
q_{l, k}: x_{1} x_{2} \ldots x_{k} x^{2 n} y^{2 n} x^{2 n} y^{2 n}=x_{1} x_{2} \ldots x_{k} y^{2 n} x^{2 n} y^{2 n}
$$

is fulfilled in $V$. Then $A_{2} \notin V$ and $A_{l}^{*} \notin V$ by Lemma 15] Let $x$ and $y$ be the first letters of the words $u^{\prime}$ and $v^{\prime}$, respectively. Since $c\left(u^{\prime}\right)=c\left(v^{\prime}\right)$, the letter $y$ occurs in $u^{\prime}$. By the condition of the case under consideration, we have $B_{0} \notin V$ and, thus, $B_{2} \notin V$. By Lemma 31 (3), we have $u^{\prime}=x^{2 n} y^{2 n} u^{\prime}=x^{2 n} y^{2 n} x^{2 n} y^{2 n} u^{\prime}$ in $F(V)$. Hence, the identity $q_{l, k}$ implies that $w_{u} u^{\prime}=w_{u} y^{2 n} x^{2 n} y^{2 n} u^{\prime}$, whence $u=w_{u} u^{\prime} w_{u}^{*}=w_{u}\left(y^{2 n} x^{2 n} y^{2 n} u^{\prime}\right) w_{u}^{*}$. Changing the notation and taking the word in the parentheses in the last identity as the new $u^{\prime}$, we obtain the representations $u=w_{u} u^{\prime} w_{u}^{*}$ and $v=w_{v} v^{\prime} w_{v}^{*}$ in which the first letters of the words $u^{\prime}$ and $v^{\prime}$ are the same. In the same way, we can arrange that the last letters of the words $u^{\prime}$ and $v^{\prime}$ become the same. Thus, it remains to consider only one case.

e) The first, as well as the last, letters of the words $u^{\prime}$ and $v^{\prime}$ coincide. The identity $u^{\prime}=v^{\prime}$ is not fulfilled in the variety $V$, because otherwise the identity $u=v$ is fulfilled in $V$, which contradicts the assumption. The words $u^{\prime}$ and $v^{\prime}$ are regular. The values of regular words in Rees-Sushkevich semigroups are regular elements (see [9]). Therefore, $u^{\prime}$ and $v^{\prime}$ are distinct regular elements of $F(V)$. By the lemma on regular elements, $u^{\prime}$ and $v^{\prime}$ can be separated by an epimorphism $\gamma$ of $F(V)$ to a completely 0-simple semigroup $S \in V$. Let $f$ be a map of the alphabet to $S$ coinciding with $\gamma$ on the set $c\left(u^{\prime}\right)=c\left(v^{\prime}\right)$ and taking all letters of the word $w_{u} \equiv w_{v}$ to a left identity of the element $\gamma\left(h\left(u^{\prime}\right)\right)$ in the semigroup $S$, all letters of the word $w_{u}^{*} \equiv w_{v}^{*}$ to a right identity of $\gamma\left(t\left(u^{\prime}\right)\right)$, and the remaining letters to arbitrary elements. This map induces a homomorphism $f: F(V) \rightarrow S$ such that $f(u)=f\left(u^{\prime}\right)=\gamma\left(u^{\prime}\right) \neq \gamma\left(v^{\prime}\right)=f\left(v^{\prime}\right)=f(v)$.

2.2. $B_{0} \in V$. If an identity $u=v$ is not fulfilled in $B_{0}$, then the elements $u$ and $v$ can be separated by a homomorphism $f: F(V) \rightarrow B_{0}$. Let $u=v$ in $B_{0}$. Then, by Proposition 6, the words $u$ and $v$ are congruent, i.e., they have the form

$$
u=w_{1} u_{1} w_{2} u_{2} \ldots w_{m} u_{m} w_{m+1}, \quad w=w_{1} v_{1} w_{2} v_{2} \ldots w_{m} v_{m} w_{m+1},
$$

where $w_{1}, w_{2}, \ldots, w_{m+1}$ are singular words, $u_{1}, \ldots, u_{m}, v_{1}, \ldots, v_{m}$ are words covered by cycles, and $c\left(u_{j}\right)=c\left(v_{j}\right)$ for all $1 \leq j \leq m$. For these representations of $u$ and $v$, we denote by $k_{*}$ the sum of the lengths of the words $w_{1}, \ldots, w_{m+1}$. We denote by $N_{h}(*)$ and $N_{t}(*)$ the sets of the indices $j$ for which the first (respectively, the last) letters of $u_{j}$ and $v_{j}$ are distinct. Let $N_{*}$ be the sum of the cardinalities of the sets $N_{h}(*)$ and $N_{t}(*)$. We assume that the representations of $u$ and $v$ are such that $k_{*}$ is the smallest possible, and $N_{*}$ is the smallest possible for this value of $k_{*}$.

Observe that, since the identity $u=v$ is not fulfilled in the variety $V$, there is $i$ such that the identity $u_{i}=v_{i}$ is not fulfilled in $V$. If $B_{2} \in V$ and the identity $u=v$ is not fulfilled in $B_{2}$, then the words $u$ and $v$ can be separated by a homomorphism $f: F(V) \rightarrow B_{2} \in V$. Therefore, we may assume that $B_{2} \notin V$ or that the identity $u=v$ is fulfilled in $B_{2}$. The latter is equivalent to the fact that the identities $u_{j}=v_{j}$ $(j=1,2, \ldots, m)$ are fulfilled in $B_{2}$.

We consider all possible cases.

2.2.1. $A_{0} \notin V$. Then the variety $V$ is regularly closed by Proposition 7 . It follows that the words $u_{j}$ and $v_{j}(1 \leq j \leq m)$ are regular elements of $F(V)$. Indeed, these words are covered by cycles and, therefore, are products of regular words, but all regular words of Rees-Sushkevich semigroups are regular elements (see [9]). Without loss of generality, we may assume that the words $u_{j}$ and $v_{j}$ themselves are regular. If $k_{*}=0$, then $u=u_{1}$ and $v=v_{1}$ are regular elements of the semigroup $F(V)$. By the lemma on 
regular elements, these elements can be separated by an epimorphism of the semigroup $F(V)$ to some completely 0 -simple semigroup $S \in V$.

If $k_{*}>0$ and $N_{*}=0$, then the first, as well as the last, letters of the words $u_{j}$ $v_{j}(j=1,2, \ldots, m)$ coincide. As we saw above, the identity $u_{i}=v_{i}$ is fulfilled in the variety $V$ for some $i$. By the lemma on regular elements, the regular words $u_{i}$ and $v_{i}$ can be separated by an epimorphism $f$ of the semigroup $F(V)$ to a completely 0 -simple semigroup $S \in V$. Let $f^{\prime}: F(V) \rightarrow S$ be the homomorphism that coincides with $f$ on the set $c\left(u_{i}\right)=c\left(v_{i}\right)$ and takes all letters in $c\left(w_{1} u_{1} w_{2} u_{2} \ldots w_{i-1}\right)$ to a left identity element of $f\left(h\left(u_{i}\right)\right)$ and all letters in $c\left(w_{i+1} u_{i+1} \ldots u_{m} w_{m}\right)$ to a right identity of the regular element $f\left(t\left(u_{i}\right)\right.$ ) (the values of $f^{\prime}$ on the remaining letters are arbitrary). Then $f^{\prime}(u)=f\left(u_{i}\right) \neq f\left(v_{i}\right)=f^{\prime}(v)$.

Now, let $N_{*}>0$. This means that there is $j$ such that the last letters of the words $u_{j}$ and $v_{j}$ are distinct. For definiteness, we assume that $h\left(u_{j}\right) \neq h\left(v_{j}\right)$. Then the identity $u=v$ is a $\bar{\lambda}$-identity. If the identity

$$
e q B_{l, n}: z^{2 n} t x^{2 n} y^{2 n} x^{2 n} y^{2 n}=z^{2 n} t y^{2 n} x^{2 n} y^{2 n}
$$

is not fulfilled in $V$, then, by Propositions 20 and 8 , the semigroup $B_{l}$ belongs to $V$. By Lemma 14, the identity $u=v$ is not fulfilled in $B_{l}$. Therefore, there is a homomorphism $f: F(V) \rightarrow B_{l}$ for which $f(u) \neq f(v)$.

Let the identity $e q B_{l, n}$ be fulfilled in $V$. If $j>1$, then the identity $u=v$ is not fulfilled in $A_{2}$; therefore, by Lemma 15, $u=v$ is not fulfilled in $A_{l}^{*}$. If $A_{2} \in V$ or $A_{l}^{*} \in V$, then the words $u$ and $v$ can be separated by a homomorphism $F(V)$ to one of the semigroups $A_{2}, A_{l}^{*} \in \mathfrak{A}_{V}$. Thus, we may assume that $A_{2}, A_{l}^{*} \notin V$. Consequently, the following identities are fulfilled in $F(V)$ by Lemma 31 (5), (6):

$$
\begin{aligned}
& u_{j-1} w_{j} u_{j}=u_{j-1} z^{2 n} w_{j} x^{2 n} y^{2 n} x^{2 n} y^{2 n} u_{j} \\
& \stackrel{e q B_{l, n}}{=} u_{j-1} z^{2 n} w_{j} y^{2 n} x^{2 n} y^{2 n} u_{j}=u_{j-1} w_{j} y^{2 n} x^{2 n} y^{2 n} u_{j},
\end{aligned}
$$

where $z=u_{j-1}^{\perp} u_{j-1}, x=u_{j} u_{j}^{\perp}$, and $y=v_{j} v_{j}^{\perp}$ (we recall that, for each regular word $U$, we denote by $U^{\perp}$ an element of the free semigroup $F(V)$ such that the $U=U U^{\perp} U$ in $F(V)$ ). In the representation $u=w_{1} u_{1} w_{2} \ldots w_{m} u_{m} w_{m+1}$, we replace the product $u_{j-1} w_{j} u_{j}$ by $u_{j-1} w_{j} u_{j}^{*}$, where $u_{j}^{*}=y^{2 n} x^{2 n} y^{2 n} u_{j}$. We obtain a representation of $u=v$ of the form (*) such that the parameter $N_{*}$ is less by 1 than that for the initial representation, which contradicts the fact that the initial representation of the form $(*)$ has the smallest possible value of $N_{*}$.

Consequently, $j=1$, i.e., the first letters of the words $u_{1}$ and $v_{1}$ are distinct. By Lemma 16, the identity $u=v$ is not fulfilled in $S L_{\left|w_{1}\right|}$. If $S L_{\left|w_{1}\right|} \in V$, then the words $u$ and $v$ can be separated by the canonical homomorphism $F(V) \rightarrow S L_{\left|w_{1}\right|} \in V$. If $S L_{\left|w_{1}\right|} \notin V$, then, by Proposition 23, one of the following identities is fulfilled in $V$ :

$$
\begin{aligned}
& q_{l,\left|w_{1}\right|}: x_{1} x_{2} \ldots x_{\left|w_{1}\right|} x^{2 n} y^{2 n} x^{2 n} y^{2 n}=x_{1} x_{2} \ldots x_{\left|w_{1}\right|} y^{2 n} x^{2 n} y^{2 n}, \\
& q_{l,\left|w_{1}\right|}^{*}: x_{1} x_{2} \ldots x_{\left|w_{1}\right|} y^{2}=x_{1} x_{2} \ldots x_{\left|w_{1}\right|}^{n+1} y^{2} .
\end{aligned}
$$

As we saw above, the second identity contradicts the minimality of $k_{*}$. Thus, the identity $q_{l,\left|w_{1}\right|}$ is fulfilled in $V$. By Lemma 15, we have $A_{2} \notin V$ and $A_{l}^{*} \notin V$. By Lemma 31, the identity $u_{1}=x^{2 n} y^{2 n} x^{2 n} y^{2 n} u_{1}$, where $x=u_{1} u_{1}^{\perp}$ and $y=v_{1} v_{1}^{\perp}$, is fulfilled in $F(V)$. Consequently,

$$
w_{1} u_{1}=w_{1} x^{2 n} y^{2 n} x^{2 n} y^{2 n} u_{1} \stackrel{q_{l,\left|w_{1}\right|}}{=} w_{1} y^{2 n} x^{2 n} y^{2 n} u_{1} .
$$

In the word $u=w_{1} u_{1} w_{2} \ldots w_{m} u_{m} w_{m+1}$, we replace the product $w_{1} u_{1}$ by $w_{1} u_{1}^{*}$, where $u_{1}^{*}=y^{2 n} x^{2 n} y^{2 n} u_{1}$, to obtain a representation of $u=v$ of the form $(*)$ with the parameter 
$N_{*}$ less by 1 than that for the initial representation $(*)$. This contradicts the fact that the initial representation of the form $(*)$ has the smallest possible value of $N_{*}$.

2.2.2. $A_{0} \in V$. If the identity $u=v$ is not fulfilled in the semigroup $A_{0}$, then the words $u$ and $v$ can be separated by a homomorphism from $F(V)$ to $A_{0}$. Therefore, we may assume that the identity $u=v$ is fulfilled in $A_{0}$. By Proposition 7 , the words $u$ and $v$ are similar. This means that, for each $i$,

$$
u_{i} \equiv u_{i, 1} u_{i, 2} \ldots u_{i, s}, \quad v_{i} \equiv v_{i, 1} v_{i, 2} \ldots v_{i, s}
$$

where the $u_{i, j}$ and $v_{i, j}$ are regular words and $c\left(u_{i, j}\right)=c\left(v_{i, j}\right)$ for all $j$.

Since the identity $u=v$ is not fulfilled in $V$, there is $i$ such that the identity $u_{i}=v_{i}$ is not fulfilled in $V$. For the representations (**) of the words $u_{i}$ and $v_{i}$, we denote by $M_{h}(* *)$ (respectively, by $\left.M_{t}(* *)\right)$ the set of indices $j$ for which the first (respectively, the last) letters of the words $u_{i, j}$ and $v_{i, j}$ are distinct. Let $M_{* *}$ be the sum of the cardinalities of the sets $M_{h}(* *)$ and $M_{t}(* *)$. We assume that the representations (**) are chosen so that $M_{* *}$ is smallest possible (for a fixed representation (*) with the smallest possible $k_{*}$ and $N_{*}$ ).

Since the identity $u_{i}=v_{i}$ is not fulfilled in $V$, there is $j$ such that the identity $u_{i, j}=v_{i, j}$ is not fulfilled in $V$. Let $M_{* *}=0$. Then the first, as well as the last, letters of the words $u_{i, j}$ and $v_{i, j}$ coincide. By the lemma of regular elements, the regular words $u_{i, j}$ and $v_{i, j}$ can be separated by an epimorphism $f$ from the semigroup $F(V)$ to a completely 0 -simple semigroup $S \in V$. The words $u$ and $v$ can be separated by a homomorphism $f^{\prime}: F(V) \rightarrow S$ that coincides with $f$ on the set $c\left(u_{i, j}\right)$ and takes all letters of the word $w_{1} u_{1} \ldots w_{i-1} u_{i-1} u_{i, 1} u_{i, 2} \ldots u_{i, j-1}$ to a left identity element of $f\left(h\left(u_{i, j}\right)\right)$ and all letters of the word $u_{i, j+1} u_{i, 2} \ldots u_{i, s} w_{i+1} u_{i+1} \ldots u_{m} w_{m+1}$ to a right identity element of $f\left(t\left(u_{i, j}\right)\right)$.

Now, let $M_{* *}>0$. Then there is $j$ such that the first or the last letters of the words $u_{i, j}$ and $v_{i, j}$ are distinct. For definiteness, we assume that the first letters of $u_{i, j}$ and $v_{i, j}$ are distinct. First, let $j>1$. If an identity of the form $e q A_{l, n}^{*}: z^{2 n} x^{2 n} y^{2 n} x^{2 n} y^{2 n}=$ $z^{2 n} y^{2 n} x^{2 n} y^{2 n}$ is not fulfilled in $V$, then, by Proposition 12, $V$ is not a $\bar{\lambda}$-variety, and we have $A_{l}^{*} \in V$ by Proposition 21. However, $u=v$ is a $\bar{\lambda}$-identity, and, by Lemma 15 , $u=v$ is not fulfilled in $A_{l}^{*}$. Therefore, the words $u$ and $v$ can be separated by a homomorphism from $F(V)$ to $A_{l}^{*} \in V$.

As before, we assume that $j>1$ and that the identity $e q A_{l, n}^{*}$ is fulfilled in $V$. Then $A_{l}^{*} \notin V$ by Lemma 15. Since $c\left(u_{i, j}\right)=c\left(v_{i, j}\right)$, Lemma 31 shows that the following identities are fulfilled in $F(V)$ :

$$
\begin{array}{r}
u_{i, j-1} u_{i, j}=u_{i, j-1} x^{2 n} y^{2 n} u_{i, j}=u_{i, j-1} z^{2 n} x^{2 n} y^{2 n} x^{2 n} y^{2 n} u_{i, j} \\
\stackrel{e q A_{l, n}^{*}}{=} u_{i, j-1} z^{2 n} y^{2 n} x^{2 n} y^{2 n} u_{i, j}=u_{i, j-1} y^{2 n} x^{2 n} y^{2 n} u_{i, j},
\end{array}
$$

where $z=u_{i, j-1}^{\perp} u_{i, j-1}, x=u_{i, j} u_{i, j}^{\perp}$, and $y=v_{i, j} v_{i, j}^{\perp}$. In the representation $u_{i} \equiv$ $u_{i, 1} u_{i, 2} \ldots u_{i, s}$, we replace $u_{i, j-1} u_{i, j}$ by $u_{i, j-1} u_{i, j}^{*}$, where $u_{i, j}^{*}=y^{2 n} x^{2 n} y^{2 n} u_{i, j}$, to obtain representations of the form $(* *)$ for the elements $u_{i}$ and $v_{i}$ such that the parameter $M_{* *}$ is less by 1 than that for the initial representation. This contradicts the fact that the initial representation of the form $(* *)$ has the smallest possible value of $M_{* *}$.

Now, let $j=1$. Then the first letters of the words $u_{i, 1}$ and $v_{i, 1}$ are distinct. Therefore, the first letters of the words $u_{i}$ and $v_{i}$ are also distinct. Consequently, $u=v$ is a $\lambda$-identity. If the identity $q_{l,\left|w_{i}\right|}: x_{1} x_{2} \ldots x_{\left|w_{i}\right|} x^{2 n} y^{2 n} x^{2 n} y^{2 n}=x_{1} x_{2} \ldots x_{\left|w_{i}\right|} y^{2 n} x^{2 n} y^{2 n}$ is fulfilled in $V$, then $A_{2} \notin V$. By Lemma[15, it follows that $A_{l}^{*} \notin V$. Since $c\left(u_{i, 1}\right)=c\left(v_{i, 1}\right)$, Lemma 31] implies that the identities

$$
w_{i} u_{i, 1}=w_{i} x^{2 n} y^{2 n} x^{2 n} y^{2 n} u_{i, 1} \stackrel{q_{l,\left|w_{i}\right|}}{=} w_{i} y^{2 n} x^{2 n} y^{2 n} u_{i, i}
$$


where $x=u_{i, 1} u_{i, 1}^{\perp}, y=v_{i, 1} v_{i, 1}^{\perp}$, are fulfilled in $F(V)$. In the representation $u=$ $w_{1} u_{1} w_{2} \ldots w_{m} u_{m} w_{m+1}$, we replace the product $w_{i} u_{i}$ by $w_{i} u_{i}^{*}$, where $u_{i}^{*}=y^{2 n} x^{2 n} y^{2 n} u_{i}$, obtaining a representation of the identity $u=v$ in the form $(*)$ such that the parameter $N_{*}$ is less by 1 than the parameter of the initial representation. This contradicts the fact that the initial representation $(*)$ has the smallest possible value of $N_{*}$.

As before, let $j=1$, and suppose that the identity $q_{l,\left|w_{i}\right|}$ is not fulfilled in $V$. As we saw above, the identity $q_{l,\left|w_{i}\right|}^{*}: x_{1} x_{2} \ldots x_{\left|w_{i}\right|} y^{2}=x_{1} x_{2} \ldots x_{\left|w_{i}\right|}^{m+1} y^{2}$ is also not fulfilled in $V$ (its validity contradicts the minimality of $k_{*}$ ). Therefore, by Proposition 23, the semigroup $S L_{\left|w_{i}\right|}$ belongs to $V$. If $i=1$, then, by Lemma 16, the identity $u=v$ is not fulfilled in $S L_{\left|w_{i}\right|}$, and the words $u$ and $v$ can be separated by a homomorphism from $F(V)$ to $S L_{\left|w_{i}\right|}$. If the identity

$$
e q B_{l, n}: z^{2 n} t x^{2 n} y^{2 n} x^{2 n} y^{2 n}=z^{2 n} t y^{2 n} x^{2 n} y^{2 n}
$$

is not fulfilled in $V$, then $B_{l} \in V$ by Propositions 20 and 8 . By assumption, $u=v$ is a $\lambda$-identity, so that $u=v$ is not fulfilled in $B_{l}$ by Lemma 14. Therefore, the words $u$ and $v$ can be separated by a homomorphism from $F(V)$ to $B_{l}$.

Finally, let $i>1$, and let the identity eq $B_{l, n}$ be fulfilled in $V$. Then $A_{2} \notin V$. Lemma 15 implies that $A_{l}^{*} \notin V$. We recall that $B_{2} \notin V$, or $B_{2} \in V$ and the identity $u_{i, j}=v_{i, j}$ is fulfilled in $B_{2} \in V$. The word $u_{i-1}$ is covered by cycles. Therefore, $u_{i-1} \equiv u_{i-1}^{\#} \widetilde{u}_{i-1}$ for some cycle $\widetilde{u}_{i-1}$. By Lemma 31, the following identities are fulfilled in $F(V)$ :

$$
\begin{aligned}
& u_{i-1} w_{i} u_{i, 1}=u_{i-1} z^{2 n} w_{i} x^{2 n} y^{2 n} x^{2 n} y^{2 n} u_{i, 1} \\
& \stackrel{e q B_{l, n}}{=} u_{i-1} z^{2 n} w_{i} y^{2 n} x^{2 n} y^{2 n} u_{i, 1}=u_{i-1} w_{i} y^{2 n} x^{2 n} y^{2 n} u_{i, 1},
\end{aligned}
$$

where $z=\widetilde{u}_{i-1}^{\perp} \widetilde{u}_{i-1}, x=u_{i, 1} u_{i, 1}^{\perp}$, and $y=v_{i, 1} v_{i, 1}^{\perp}$. In $u=w_{1} u_{1} w_{2} \ldots w_{m} u_{m} w_{m+1}$, we replace the product $u_{i-1} w_{i} u_{i}$ by $u_{i-1} w_{i} u_{i}^{*}$, where $u_{i}^{*}=y^{2 n} x^{2 n} y^{2 n} u_{i}$, to obtain a representation of the form $(*)$ for $u=v$ such that the parameter $N_{*}$ is less by 1 than that for the initial representation. This contradicts the fact that the initial representation (*) has the smallest possible value of $N_{*}$.

Theorem 1 is proved completely.

Proof of Theorem 2, Let $V_{1}$ and $V_{2}$ be semisimple Rees-Sushkevich varieties such that $V_{1}^{\prime}=V_{2}^{\prime}$ and $\operatorname{Rank}\left(V_{1}\right)=\operatorname{Rank}\left(V_{2}\right)$. We must prove that $V_{1}=V_{2}$.

We recall that $C S^{0} V$ is the class of all completely 0-simple semigroups in $V, \mathfrak{N}_{V}$ is the set of finite semigroups in $\mathfrak{R}(V)$, and $\mathfrak{A}_{V}=\mathfrak{A} \cap V$. By Theorem 11, we have

$$
V_{1}=\operatorname{Var}\left(C S^{0} V_{1}, \mathfrak{A} \cap V_{1}, \mathfrak{N}_{V_{1}}\right), \quad V_{2}=\operatorname{Var}\left(C S^{0} V_{2}, \mathfrak{A} \cap V_{2}, \mathfrak{N}_{V_{2}}\right) .
$$

However, $C S^{0} V_{1}=C S^{0} V_{2}$ and, therefore, $V_{1}^{\prime}=V_{2}^{\prime}$. We see that $\mathfrak{N}_{V_{1}}=\mathfrak{N}_{V_{2}}=0$, because the varieties $V_{1}$ and $V_{2}$ are semisimple. Consequently, $\mathfrak{R}\left(V_{1}\right)=\mathfrak{R}\left(V_{2}\right)=0$. Finally, $\mathfrak{A}_{V_{1}}=\mathfrak{A}_{V_{2}}$ because $\operatorname{Rank}\left(V_{1}\right)=\operatorname{Rank}\left(V_{2}\right)$. Thus, $V_{1}=V_{2}$.

Proof of Theorem 3. We must prove that every Rees-Sushkevich variety $V$ can be represented as the sum of the radical and a semisimple variety. By Theorem 1, we have

$$
V=\operatorname{Var}\left(C S^{0} V, \mathfrak{A}_{V}, \mathfrak{N}_{V}\right)=\operatorname{Var}\left(\mathfrak{N}_{V}\right)+\operatorname{Var}\left(\mathfrak{A}_{V}\right)+V^{\prime} .
$$

If $\mathfrak{R}(V)=0$, then $V$ is a semisimple variety. Let $\mathfrak{R}(V) \neq 0$. By Lemma 25, we obtain

$$
\mathfrak{R}(V)=V \cap\left[x^{2}=0, x y x=0\right] .
$$

It is clear that all finitely generated semigroups in $\left[x^{2}=0, x y x=0\right]$ and, therefore, also in $\mathfrak{R}(V) \subseteq\left[x^{2}=0, x y x=0\right]$, are finite. Every variety is generated by its finitely generated semigroups. Therefore, the variety $\mathfrak{R}(V)$ is generated by the set $\mathfrak{N}_{V}$ of its 
finite semigroups. Thus, $\operatorname{Var}\left(\mathfrak{N}_{V}\right)=\mathfrak{R}(V)$ is a radical variety. We put $\mathfrak{D}_{V}=\left\{C N_{m} \mid m \in\right.$ $\mathbb{N}\} \cap V$. Clearly, we have $\mathfrak{D}_{V} \subset \mathfrak{N}(V)$, so that

$$
\operatorname{Var}(V)=\operatorname{Var}\left(\mathfrak{N}_{V}\right)+\operatorname{Var}\left(\mathfrak{A}_{V} \backslash \mathfrak{D}_{V}\right)+V^{\prime} .
$$

As we saw above, $\operatorname{Var}\left(\mathfrak{N}_{V}\right)$ is a radical variety. Thus, it remains to prove that the variety $\operatorname{Var}\left(\mathfrak{A}_{V} \backslash \mathfrak{D}_{V}\right)+V^{\prime}$ is semisimple, i.e., that if a permutation identity is fulfilled in $\operatorname{Var}\left(\mathfrak{A}_{V} \backslash \mathfrak{D}_{V}\right)+V^{\prime}$, then $k \geq n_{D}\left(\operatorname{Var}\left(\mathfrak{A}_{V} \backslash \mathfrak{D}_{V}\right)+V^{\prime}\right)$. Indeed, assuming the contrary, let a permutation identity of degree $k$ be fulfilled in $\operatorname{Var}\left(\mathfrak{A}_{V} \backslash \mathfrak{D}_{V}\right)+V^{\prime}$, and let $k<$ $n_{D}\left(\operatorname{Var}\left(\mathfrak{A}_{V} \backslash \mathfrak{D}_{V}\right)+V^{\prime}\right)$. We recall that

$$
\mathfrak{A}_{V} \backslash \mathfrak{D}_{V} \subseteq\left\{A_{0}, B_{0}, B_{l}, A_{l}^{*}, B_{r}, A_{r}^{*}, S L_{m}, S R_{m}, S L_{m}^{*}, S R_{m}^{*}, N_{m} \mid m \in \mathbb{N}\right\} .
$$

From Propositions 7 and 6 and Lemmas 14 and 15, it follows that there are no permutation identities valid in $A_{0}, B_{0}, B_{l}, A_{l}^{*}$, or $B_{r}, A_{r}^{*}$. Therefore, these subgroups do not belong to $\mathfrak{A}_{V} \backslash \mathfrak{D}_{V}$ and, thus,

$$
\operatorname{Var}\left(\mathfrak{A}_{V} \backslash \mathfrak{D}_{V}\right)+V^{\prime} \subseteq \operatorname{Var}\left(\left\{S L_{m}, S R_{m}, S L_{m}^{*}, S R_{m}^{*}, N_{m} \mid m \in \mathbb{N}\right\} \cup C S^{0} V\right) .
$$

Lemmas [16, 17, and 26] show that if the permutation identity in question is fulfilled in the semigroup $S \in\left\{S L_{m}, S R_{m}, S L_{m}^{*}, S R_{m}^{*} \mid m \in \mathbb{N}\right\} \cup C S^{0} V$, then the nonpermutation identity $x_{1} x_{2} \ldots x_{k}=\left(x_{1} x_{2} \ldots x_{k}\right)^{n+1}$ (here $n$ is a number such that $V \subset R S_{n}$ ) is fulfilled in $S$. It is easily seen that the same is true for the semigroups $S \in\left\{N_{m} \mid m \in \mathbb{N}\right\}$. Thus, a nonpermutation identity of the form $x_{1} \ldots x_{k}=w$ is fulfilled in the variety $\operatorname{Var}\left(\mathfrak{A}_{V} \backslash \mathfrak{D}_{V}\right)+V^{\prime}$. However, by the definition of the degree of a variety, this is impossible if $k<n_{D}\left(\operatorname{Var}\left(\mathfrak{A}_{V} \backslash \mathfrak{D}_{V}\right)+V^{\prime}\right)$.

Proof of Theorem 4, Let $V$ be a Rees-Sushkevich variety of period $n$. We must prove that $V$ can be defined by the set of identities $\left(\mathrm{I}_{\mathrm{n}}\right),\left(\mathrm{II}_{\mathrm{n}}\right),\left(\mathrm{III}_{\mathrm{n}}\right)$, and a certain set of identities in $E q R S[n]$. Let $I(V)$ be the set of identities of the form indicated above that are fulfilled in $V$, and let $V^{\#}=[I(V)]$. It is clear that $V \subset V^{\#}$. We prove the reverse inclusion $V^{\#} \subset V$. By Theorem 1] we have

$$
V^{\#}=\operatorname{Var}\left(C S^{0} V^{\#}, \mathfrak{A}_{V \#}, \mathfrak{N}_{V^{\#}}\right), \quad V=\operatorname{Var}\left(C S^{0} V, \mathfrak{A}_{V}, \mathfrak{N}_{V}\right) .
$$

Therefore, it suffices to prove that

$$
C S^{0} V^{\#} \subseteq C S^{0} V, \quad \mathfrak{A}_{V^{\#}} \subseteq \mathfrak{A}_{V}, \quad \mathfrak{N}_{V^{\#}} \subseteq \mathfrak{N}_{V} .
$$

Let $K \in C S^{0} V^{\#}$. If $K \notin C S^{0} V$, then there is an identity $u=v$ fulfilled in $V$ but not in $K$. Let $u^{f}=v^{f}$ be the identity obtained from $u=v$ by the replacement of each letter $x$ by the word $x y_{x} t z_{x} x$, where $t, y_{x}$, and $z_{x}$ are letters that do not occur in the words $u$ and $v$. The identity $u^{f}=v^{f}$ has the form Eqreg and it is fulfilled in $V$ because this identity is a consequence of $u=v$. Therefore, $u^{f}=v^{f}$ belongs to $I(V)$. However, by Lemma 27, the identity $u^{f}=v^{f}$ is not fulfilled in $K$. Consequently, $K \notin[I(V)]=V^{\#}$, and a fortiori $K \notin C S^{0} V^{\#}$, which contradicts the assumption. Thus, $C S^{0} V^{\#} \subseteq C S^{0} V$.

Now we prove that $\mathfrak{A}_{V \#} \subseteq \mathfrak{A}_{V}$. For this, we must prove that, for each semigroup $S \in \mathfrak{A}$, if $S \notin V$, then there is an identity in $I(V)$ that is not fulfilled in $S$. In this case, $S \notin[I(V)]=V^{\#}$.

1. If $B_{0} \notin V$, then $e q_{2} \in I(V)$ by Proposition 6 . Then the identity $e q_{2, n}$ belongs to $I(V)$ by Lemma 43. Therefore, $e q_{2, n}$ is not fulfilled in $B_{0}$.

2. If $A_{0} \notin V$, then $e q_{1} \in I(V)$ by Proposition 7 . Then the identity $e q_{1, n}$ belongs to $I(V)$ by Lemma 43. Therefore, $e q_{1, n}$ is not fulfilled in $A_{0}$.

3. If $B_{l} \notin V$, then the identity

$$
e q B_{l, n}: z^{2 n} t x^{2 n} y^{2 n} x^{2 n} y^{2 n}=z^{2 n} t y^{2 n} x^{2 n} y^{2 n}
$$

belongs to $I(V)$ by Propositions 20 and 8 . This identity is not fulfilled in $B_{l}$. 
4. If $A_{l}^{*} \notin V$, then the identity

$$
e q A_{l, n}^{*}: z^{2 n} x^{2 n} y^{2 n} x^{2 n} y^{2 n}=z^{2 n} y^{2 n} x^{2 n} y^{2 n}
$$

belongs to $I(V)$ by Propositions 21 and 12. This identity is not fulfilled in $A_{l}^{*}$.

5. If $S L_{k}^{*} \notin V$, then the identity

$$
q_{l, k}^{*}: x_{1} x_{2} \ldots x_{k} y^{2}=x_{1} x_{2} \ldots x_{k}^{m+1} y^{2}
$$

belongs to $I(V)$ by Proposition 24. This identity is not fulfilled in $S L_{k}^{*}$.

6. If $S L_{k} \notin V$, then, by Proposition [23, one of the identities $q_{l, k}^{*}$ or

$$
q_{l, k}: x_{1} x_{2} \ldots x_{k} x^{2 n} y^{2 n} x^{2 n} y^{2 n}=x_{1} x_{2} \ldots x_{k} y^{2 n} x^{2 n} y^{2 n}
$$

belongs to $I(V)$. None of these identities is contained in $S L_{k}$.

7. If $N_{k} \notin V$, then, by Lemma 2, an identity of the form $x_{1} x_{2} x_{3} \ldots x_{k}=w$, where $w \not \equiv x_{1} x_{2} x_{3} \ldots x_{k}$ and $|w| \geq k$, is fulfilled in the variety $V$.

If this identity is a permutation, then it belongs to $I(V)$ but is not fulfilled in $N_{k}$. If the identity in question is a nonpermutation, then $w$ either contains a letter that occurs more than once, or it contains letters distinct from $x_{1}, x_{2}, \ldots, x_{k}$. In any case, there is a consequence $x_{1} x_{2} x_{3} \ldots x_{k}=\widetilde{w}$ of the identity $x_{1} x_{2} x_{3} \ldots x_{k}=w$ such that $|\widetilde{w}|>k$. By Lemma 29, an identity of the form

$$
E q D^{(n)}: x_{1} \ldots x_{l} z_{1} \ldots z_{m} y_{1} \ldots y_{r}=x_{1} \ldots x_{l}\left(z_{1} \ldots z_{m}\right)^{n+1} y_{1} \ldots y_{r}
$$

with $l+m+r=k$ and $m>0$ is fulfilled in the variety $V$. This identity belongs to $I(V)$ but is not fulfilled in $N_{k}$.

8. Let $C N_{k} \notin V$. Lemma 3 implies that an identity of the form $x_{1} x_{2} x_{3} \ldots x_{k}=w$ with $|w|>k$ is fulfilled in $V$. By Lemma 29, an identity of the form

$$
E q D^{(n)}: x_{1} \ldots x_{l} z_{1} \ldots z_{m} y_{1} \ldots y_{r}=x_{1} \ldots x_{l}\left(z_{1} \ldots z_{m}\right)^{n+1} y_{1} \ldots y_{r}
$$

with $l+m+r=k$ and $m>0$ is fulfilled in the variety $V$. This identity belongs to $I(V)$ but is not fulfilled in $C N_{k}$.

By duality, we can show that if one of the semigroups $B_{r}, A_{r}^{*}, S R_{k}^{*}$, or $S R_{k}$ does not belong to $V$, then there is an identity in $I(V)$ that is not fulfilled in this semigroup.

Remark 6. The above argument shows, in particular, that if $V \subset R S_{n}$, then the set $\mathfrak{A}_{V}$ is uniquely determined by the identities in $E q R S_{n}$ and $E q N$ that are fulfilled in $V$. This observation will be used in the proof of Theorem 7 .

It remains to prove that $\mathfrak{R}\left(V^{\#}\right)=\mathfrak{R}(V)$. We note that $V$ and $V^{\#}$ are varieties of the same degree. Indeed, if $V$ is a variety of infinite degree, then so is $V^{\#}$, because $V \subset V^{\#}$. If $C N_{k}$ belongs to $V^{\#}$ for some $k$, then $C N_{k} \in V$ (see item 8 above). This means that $n_{D}\left(V^{\#}\right)=n_{D}(V)$. If a permutation identity is fulfilled in $V$, then, by definition, this identity belongs to the set $I(V)$; therefore, this identity is fulfilled in the variety $V^{\#}=V[I(V)]$. Thus, the varieties $\mathfrak{R}\left(V^{\#}\right)$ and $\mathfrak{R}(V)$ are defined by the same set of identities, i.e., these varieties coincide. Therefore, the sets $\mathfrak{N}_{V \#}$ and $\mathfrak{N}_{V}$ also coincide.

Theorem 4 is proved completely.

Proof of Theorem 5. Theorems 1 and 4 and the definitions show immediately that if $V_{1}$ and $V_{2}$ are Rees-Sushkevich varieties with $V_{1}^{\prime}=V_{2}^{\prime}$ and $\operatorname{Rank}\left(V_{1}\right)=\operatorname{Rank}\left(V_{2}\right)$, then there exist bases of identities for these varieties that differ only in the sets of permutation identities, which is exactly the claim of Theorem 5 .

Proof of Corollary 1 (Exactness criterion). Let $V$ be a Rees-Sushkevich variety. We must prove that $V$ is exact (i.e., $V$ is generated by 0 -simple semigroups) if and only if one of the following conditions is fulfilled:
(C1) $\quad A_{2} \in V$;
(C2) $\quad N_{1} \notin V$; 
(C3) $\quad B_{2} \in V, A_{0} \notin V$ and $M_{p} \in V$ for a prime $p$;

(C4) $\quad B_{2} \in V$ and $A_{0}, S L_{1}, S R_{1} \notin V$.

Let $V$ be an exact variety of period $n$. We assume that condition $(C 1)$ fails, i.e., $A_{2} \notin V$. Then Lemma 31 implies that the identity $a^{2 n} b^{2 n}=\left(a^{2 n} b^{2 n}\right)^{n+1}$ is fulfilled in all completely 0 -simple semigroups in $V$. Thus, this identity is also fulfilled in $V$. By Proposition 7 , the semigroup $A_{0}$ does not belong to $V$. We consider all possible cases.

1. $B_{2} \notin V$. In this case, all completely 0 -simple semigroups in $V$ are completely simple, possibly, with zero adjoined. Therefore, the identity $x=x^{n+1}$ is fulfilled in these semigroups. Since an exact variety $V$ is generated by its completely 0 -simple semigroups, this identity is fulfilled in $V$. However, the identity in question is not fulfilled in the semigroup $N_{1}$. Consequently, $N_{1} \notin V$. Thus, condition $(C 2)$ is fulfilled in the case in question.

2. $B_{2} \in V$ and $M_{p} \in V$ for some $p$. We already know that $A_{0} \notin V$. Therefore, condition $(C 3)$ is fulfilled.

3. $B_{2} \in V$ and $M_{p} \notin V$ for all $p$. In this case, all completely 0 -simple semigroups in $V$ must be orthodox, and, therefore, the identity $a x^{2 n} y^{2 n} b=a y^{2 n} x^{2 n} b$ is fulfilled in these semigroups. Since every exact variety $V$ is generated by its completely 0 -simple semigroups, this identity is fulfilled in $V$. Since the same identity is not fulfilled in the semigroups $S L_{1}$ and $S R_{1}$ by Lemma [16, we have $S L_{1}, S R_{1} \notin V$, i.e., condition $(C 4)$ is satisfied.

Conversely, we prove that if $V$ is a Rees-Sushkevich variety satisfying one of conditions $(C 1)-(C 4)$, then the variety $V$ is exact. By Theorem 1, we have $V=\operatorname{Var}\left(C S^{0} V, \mathfrak{A}_{V}, \mathfrak{N}_{V}\right)$.

1. Let condition $(C 1)$ be fulfilled, i.e., $A_{2} \in V$. We note that $\operatorname{Var}\left(A_{2}\right)=R S_{1}$. By the definitions and Lemma 5, we have $\mathfrak{A}_{V}, \mathfrak{N}_{V} \subset R S_{1}=\operatorname{Var}\left(A_{2}\right) \subset \operatorname{Var}\left(C S^{0} V\right)$. Thus, $V=\operatorname{Var}\left(C S^{0} V, \mathfrak{A}_{V}, \mathfrak{N}_{V}\right)=\operatorname{Var}\left(C S^{0} V\right)$ is an exact variety.

2. If condition $(C 2)$ is fulfilled, i.e., $N_{1} \notin V$, then the identity $x=x^{n+1}$ is fulfilled in $V$. By Lemma 30, the variety $V$ is generated by its completely simple semigroups.

3. Assume that condition $(C 3)$ is fulfilled but condition $(C 1)$ is not, i.e., $A_{0}, A_{2} \notin V$, $B_{2}, M_{p} \in V$ for a prime $p$. Since $A_{2} \notin V$, the identity $a^{2 n} b^{2 n}=\left(a^{2 n} b^{2 n}\right)^{n+1}$ is fulfilled in all completely 0 -simple semigroups in $V$ by Lemma 31. We observe that $B_{l} \in \operatorname{Var}\left(B_{2}, M_{p}\right)$, because, otherwise the identity $z^{2 n} t x^{2 n} y^{2 n} x^{2 n} y^{2 n}=z^{2 n} t y^{2 n} x^{2 n} y^{2 n}$ is fulfilled in $\operatorname{Var}\left(B_{2}, M_{p}\right)$ by Propositions 20 and 8 . However, this identity is not fulfilled in $M_{p}$. By duality, we can prove that $B_{r} \in \operatorname{Var}\left(B_{2}, M_{p}\right)$.

Next, $S L_{k} \in \operatorname{Var}\left(B_{2}, M_{p}\right)$ for each $k$, because otherwise one of the identities

$$
x_{1} \ldots x_{k} x^{2 n} y^{2 n} x^{2 n} y^{2 n}=x_{1} \ldots x_{k} y^{2 n} x^{2 n} y^{2 n}, \quad x_{1} \ldots x_{k} y^{2}=x_{1} \ldots x_{k}^{m+1} y^{2}
$$

is fulfilled in $\operatorname{Var}\left(B_{2}, M_{p}\right)$ by Proposition 23. However, the former is not fulfilled in $M_{p}$, and the latter is not fulfilled in $B_{2}$. By duality, we can prove that $S R_{k} \in \operatorname{Var}\left(B_{2}, M_{p}\right)$ for each $k$.

By Lemma 32, we have $\left[x^{2}=0, x y x=0\right] \subset \operatorname{Var}\left(B_{0}\right)$. Since $B_{0}$ is a subsemigroup of $B_{2}$, we see that $N_{k}$ and $C N_{k}$ are contained in $\operatorname{Var}\left(B_{2}\right)$. Thus, we have established that $\mathfrak{A}_{V} \cup \mathfrak{N}_{V} \subset \operatorname{Var}\left(B_{2}, M_{p}\right) \subset \operatorname{Var}\left(C S^{0} V\right)$; thus, $V=\operatorname{Var}\left(C S^{0} V, \mathfrak{A}_{V}, \mathfrak{N}_{V}\right)=\operatorname{Var}\left(C S^{0} V\right)$ is an exact variety.

4. Let condition $(C 4)$ be fulfilled, i.e., $B_{2} \in V$ and $A_{0}, S L_{1}, S R_{1} \notin V$. Since $S L_{1} \notin V$, Lemma 23 shows that one of the identities

$$
x_{1} x^{2 n} y^{2 n} x^{2 n} y^{2 n}=x_{1} y^{2 n} x^{2 n} y^{2 n}, \quad x y^{2}=x^{m+1} y^{2}
$$

is fulfilled in $V$. The second identity is not fulfilled in $B_{2} \in V$. Therefore, the first identity is fulfilled in $V$. By Lemma 15, the first identity is not fulfilled in $A_{l}^{*}$. Thus, $A_{l}^{*} \notin V$. By duality, we can prove that $A_{r}^{*} \notin V$. 
We have $S L_{k}^{*} \in V\left(B_{2}\right)$ for each $k$, because otherwise the identity $x_{1} x_{2} \ldots x_{k} y^{2}=$ $x_{1} x_{2} \ldots x_{k}^{m+1} y^{2}$, which is not fulfilled in $B_{2}$, must be fulfilled in $V\left(B_{2}\right)$ by Proposition 24. By Lemma 32, we have

$$
\left[x^{2}=0, x y x=0\right] \subset \operatorname{Var}\left(B_{0}\right) \subset \operatorname{Var}\left(B_{2}\right) .
$$

It follows that $\mathfrak{N}_{V} \subset \operatorname{Var}\left(B_{2}\right)$ and that $N_{k}$ and $C N_{k}$ are contained in $\operatorname{Var}\left(B_{2}\right)$ for every $k$. Thus,

$$
\mathfrak{A}_{V}=\left\{B_{0}, S L_{1}^{*}, S L_{1}^{*}\right\} \cup\left\{N_{k}, C N_{k} \mid k \in \mathbb{N}\right\} \subset \operatorname{Var}\left(B_{2}\right) .
$$

Therefore, $\mathfrak{A}_{V} \cup \mathfrak{N}_{V} \subset \operatorname{Var}\left(B_{2}\right) \subset \operatorname{Var}\left(C S^{0} V\right)$, and, consequently,

$$
V=\operatorname{Var}\left(C S^{0} V, \mathfrak{A}_{V}, \mathfrak{N}_{V}\right)=\operatorname{Var}\left(C S^{0} V\right)
$$

is an exact variety.

Proof of Corollary 2. Let $V$ be a finitely based periodic variety of semigroups of period $n$. We must prove that $V^{\prime}$ is finitely based. Let $\left\{u_{i}=v_{i} \mid i\right\}$ be a finite basis of identities of $V$. Since $V^{\prime}$ is exact by definition, the exactness criterion (Corollary 1) implies that one of conditions $(C 1)-(C 4)$ is fulfilled for $V^{\prime}$. We consider each of these conditions separately.

$(C 1): A_{2} \in V^{\prime}$. It is clear that $A_{2} \in V \cap R S_{n}$. Therefore, the exactness criterion shows that $V \cap R S_{n}=\operatorname{Var}\left(C S^{0}\left(V \cap R S_{n}\right)\right)$ is an exact variety. This variety is contained in the variety $V^{\prime}$ generated by the larger class $C S^{0} V$ of semigroups. On the other hand, $V^{\prime}$ is contained in each of the varieties $V$ and $R S_{n}$. Therefore, $V^{\prime} \subseteq V \cap R S_{n}$. Thus, $V^{\prime}=V \cap R S_{n}$, and the identities $\left\{u_{i}=v_{i} \mid i\right\},(\mathrm{I})_{n},(\mathrm{II})_{n}$, and (III) $)_{n}$ constitute a finite basis of $V^{\prime}$.

$(C 2): N_{1} \notin V^{\prime}$. Then all semigroups in $C S^{0} V$ are completely simple, possibly, with zero adjoined. Therefore, the identity $x=x^{n+1}$ is fulfilled in $V^{\prime}=\operatorname{Var}\left(C S^{0} V\right)$. Consequently, $V^{\prime} \subseteq V^{+}=V \cap R S_{n} \cap\left[x=x^{n+1}\right]$. The semigroup $N_{1}$ does not belong to $V^{+}$. Therefore, by Corollary 1, the variety $V^{+}$is exact, i.e., $V^{+}=\operatorname{Var}\left(C S^{0} V^{+}\right)$. However, $V^{\prime}$ is generated by the larger class $C S^{0} V$ of semigroups. Thus, $V^{+} \subseteq V^{\prime}$. Therefore, $V^{\prime}=V^{+}=V \cap R S_{n} \cap\left[x=x^{n+1}\right]$, and the finite set of identities $\left\{u_{i}=v_{i} \mid i\right\},(\mathrm{I})_{n},(\mathrm{II})_{n}$, (III) $)_{n}$, and $x=x^{n+1}$ forms a basis of identities in $V^{\prime}$.

$(C 3): A_{0} \notin V^{\prime}$ and $B_{2}, M_{p} \in V^{\prime}$ for some $p$. Since the case of $(C 1)$ has already been settled, without loss of generality we may assume that condition $(C 1)$ is not fulfilled in $V^{\prime}$, i.e., $A_{2} \notin V^{\prime}$. Then Lemma 31 implies that the identity $a^{2 n} b^{2 n}=\left(a^{2 n} b^{2 n}\right)^{n+1}$ is fulfilled in all completely 0 -simple semigroups in $V^{\prime}$. By Proposition 7 , this identity is not fulfilled in the semigroup $A_{0}$. Therefore, $A_{0} \notin V^{+}=V \cap R S_{n} \cap\left[a^{2 n} b^{2 n}=\left(a^{2 n} b^{2 n}\right)^{n+1}\right]$. However, the semigroups $B_{2}$ and $M_{p}$ are contained in $V^{+}$, because $V^{+}=\operatorname{Var}\left(C S^{0} V^{+}\right)$is an exact variety by the exactness criterion (Corollary 1). The variety $V^{\prime}$ is generated by the larger class $C S^{0} V$ of semigroups, whence $V^{+} \subseteq V^{\prime}$. The reverse inclusion is obvious. Hence, $V^{\prime}=V^{+}=V \cap R S_{n} \cap\left[a^{2 n} b^{2 n}=\left(a^{2 n} b^{2 n}\right)^{n+1}\right]$, and the finite set of identities $\left\{u_{i}=v_{i} \mid i\right\},(\mathrm{I})_{n},(\mathrm{II})_{n},(\mathrm{III})_{n}$, and $a^{2 n} b^{2 n}=\left(a^{2 n} b^{2 n}\right)^{n+1}$ is a basis of identities in the variety $V^{\prime}$.

$(C 4): B_{2} \in V^{\prime}$ and $A_{0}, S L_{1}, S R_{1} \notin V^{\prime}$. As in the previous case, without loss of generality we may assume that conditions $(C 1),(C 2)$, and $(C 3)$ are not fulfilled in $V^{\prime}$. In this case, all completely 0 -simple semigroups in $V$ must be orthodox, and, therefore, the identity $a x^{2 n} y^{2 n} b=a y^{2 n} x^{2 n} b$ is fulfilled in these semigroups. This identity is not fulfilled in the semigroups $A_{0}, S L_{1}$, and $S R_{1}$. Therefore, these semigroups do not belong to the variety $V^{+}=V \cap R S_{n} \cap\left[a x^{2 n} y^{2 n} b=a y^{2 n} x^{2 n} b\right]$. Since, obviously, $B_{2} \in V^{+}$, the exactness criterion shows that the variety $V^{+}=\operatorname{Var}\left(C S^{0} V^{+}\right)$is exact. The variety $V^{\prime}$ is generated by the larger class $C S^{0} V$ of semigroups. Therefore, $V^{+} \subseteq V^{\prime}$. The reverse inclusion is obvious, so that $V^{\prime}=V^{+}=V \cap R S_{n} \cap\left[a x^{2 n} y^{2 n} b=a y^{2 n} x^{2 n} b\right]$. Thus, the 
finite set of identities $\left\{u_{i}=v_{i} \mid i\right\},(\mathrm{I})_{n},(\mathrm{II})_{n},(\mathrm{III})_{n}$, and $a x^{2 n} y^{2 n} b=a y^{2 n} x^{2 n} b$ is a basis of identities in the variety $V^{\prime}$.

Proof of Corollary 3 (Description of the exact combinatorial varieties). Let $V \subset R S_{1}$ be an exact combinatorial variety. We must prove that $V$ coincides with one of the varieties listed in the statement of the corollary. As every exact variety, $V$ is generated by a set of completely 0 -simple semigroups. Without loss of generality, we may assume that $V$ is generated by a single completely 0 -simple semigroup, namely, by the 0-direct product of the completely 0-simple semigroups generating $V$. If $A_{2} \in V$, then $V=\operatorname{Var}\left(A_{2}\right)=R S_{1}$. If $A_{2} \notin V$, then $V$ is generated by a Rees matrix semigroup with a block-diagonal sandwich-matrix whose nonzero entries are equal to 1 . Then the variety $V$ is generated by a set of semigroups belonging to $\left\{Y, L_{2}, R_{2}, B_{2}\right\}$, and we can use the exactness criterion.

Proof of Corollary 4 (A criterion for a subgroup to be finitely based). Let $V$ be a ReesSushkevich variety. We must prove that $V$ is finitely based (hereditarily finitely based) if and only if the variety $V^{\prime}$ has the same property. The "only if" part follows from Corollary [2. To prove the "if" part, assume that the variety $V^{\prime}$ is finitely based. By Lemma 28, the set $\operatorname{Eqreg}(V)$ of identities has a finite basis. By Proposition 34, every set of permutation identities has a finite basis. Theorem 4 shows that $V$ has a finite basis of identities. The claim concerning the criterion for a variety to be hereditarily finitely based can be proved similarly (we recall that a variety $V$ is said to be hereditarily finitely based if every subvariety of $V$ is finitely based).

Proof of Corollary 5. Let $S$ be a Rees-Sushkevich semigroup. By Proposition 41, we have $(\operatorname{Var}(S))^{\prime}=\operatorname{Var}\left(S^{\prime}\right)$. Therefore, the preceding corollary implies that the semigroup $S$ is finitely based (hereditarily finitely based) if and only if its derivative semigroup $S^{\prime}$ is finitely based (hereditarily finitely based), which is precisely the statement of Corollary 5 .

Proof of Corollary 6. We must prove that a finite orthodox Rees-Sushkevich semigroup is hereditarily finitely based. By the theorem proved in [8] by Oates and Powell, a variety generated by a finite group is hereditarily finitely based. It follows (see [6]) that a variety generated by a finite orthodox completely 0-simple semigroup is hereditarily finitely based. Therefore, the semigroup itself is finitely based by the preceding corollary.

Proof of Corollary 7. We must prove that the variety $R S_{1}$ is hereditarily finitely based. By Corollary [3, the variety $R S_{1}$ has only a finite number of exact subvarieties, and all such subvarieties are finitely based. Thus, the variety $\left(R S_{1}\right)^{\prime}$ is hereditarily finitely based. By Corollary 4 , the variety $R S_{1}$ is also hereditarily finitely based.

Proof of Corollary 8 . Let $V$ be a Rees-Sushkevich variety. We must prove that every interval $\left[V^{\prime}, V\right]$ of the lattice of subvarieties of $V$ satisfies the minimality condition and has no infinite antichains.

Let $V_{1} \supset V_{2} \supset \cdots \supset V_{i} \supset \cdots$ be an infinite descending chain in the interval $\left[V^{\prime}, V\right]$. We note that $V^{\prime}=V_{1}^{\prime}=V_{2}^{\prime}=\cdots=V_{i}^{\prime}=\cdots$. Then, starting with some index, the decreasing ranks of the varieties $V_{j}$ stabilize, i.e., there is an index $i$ such that $\operatorname{Rank}\left(V_{i}\right)=\operatorname{Rank}\left(V_{i+1}\right)=\cdots$. By Theorem [5, the varieties $V_{j}, j \geq i$, have bases of identities $E T\left(V_{i}\right)$ that differ only in permutation identities. Thus, each set of identities in the chain $E T\left(V_{i}\right) \subset E T\left(V_{i+1}\right) \subset \cdots$ does not follow from the preceding one, but is obtained from it by appending permutation identities. Hence, we obtain an infinite set of permutation identities without a finite basis, which contradicts Proposition 34 . 
Let $\left\{V_{i}\right\}_{i \in \mathbb{N}}$ be an infinite antichain in the interval $\left[V^{\prime}, V\right]$. We note that $V^{\prime}=V_{1}^{\prime}=$ $V_{2}^{\prime}=\cdots$. By Theorem 3 , every Rees-Sushkevich variety $V_{i}$ decomposes into the sum of a radical variety $\mathfrak{R}_{i}$ and a semisimple variety $W_{i}$ such that $W_{i}^{\prime}=V_{i}^{\prime}=V^{\prime}$.

There are no infinite antichains or infinite descending chains in the family $\left\{W_{i}\right\}_{i \in \mathbb{N}}$. Indeed, assume that such an antichain exists. Since all $W_{i}$ are semisimple and their derivatives coincide, Theorem 2 implies that the ranks of the varieties in the chain are pairwise incomparable, and the ranks of the varieties in the infinite decreasing antichain form an infinite decreasing chain. However, this is impossible because, by Lemma 33 , the 12 th power of the set of positive integers with zero and infinity appended contains no infinite antichains nor infinite descending chains. Consequently, by the same Lemma 33. the family $\left\{W_{i}\right\}_{i \in \mathbb{N}}$ has an infinite increasing subfamily. Discarding all $W_{j}$ that do not belong to this subfamily and changing enumeration, without loss of generality we may assume that $W_{1} \subset W_{2} \subset \cdots$. By Proposition [36, the family $\left\{\mathfrak{R}_{i}\right\}_{i \in \mathbb{N}}$ forms a well ordered set, and, by Lemma 33 , $\left\{\mathfrak{R}_{i}\right\}_{i \in \mathbb{N}}$ has an increasing subfamily $\left(\mathfrak{R}_{i_{k}}\right)_{k \in \mathbb{N}}$. Thus, $V_{i_{1}}=\mathfrak{R}_{i_{1}}+W_{i_{1}} \subset \mathfrak{R}_{i_{2}}+W_{i_{2}}=V_{i_{2}}$, which contradicts the fact that $\left\{V_{i}\right\}_{i \in \mathbb{N}}$ is an antichain.

Proof of Corollary 9. We must prove that a Rees-Sushkevich variety $V$ satisfies the minimality condition or the finiteness condition for antichains if the same is true for the derivative variety $V^{\prime}$. This follows immediately from Corollaries 3 and 8 .

In particular, the variety $R S_{1}$ satisfies the minimality condition and the finiteness conditions for antichains. Therefore, every variety in $R S_{1}$ has a finite number of covers.

Proof of Corollary 10, Let $V$ be a Rees-Sushkevich variety. We must prove that the width of $V$ is finite if and only if the width of $V^{\prime}$ is finite and either $V$ is a diverse variety, or a permutation identity is fulfilled in $V$. The "only if" part was proved in the paper 2] by Sapir and Sukhanov. We prove the "if" part.

If a permutation identity is fulfilled in $V$, then, by the result of [13], the variety $V$ has only a finite number of subvarieties, so that the width of $V$ is finite. Now, let $d$ be the finite width of the variety $V^{\prime}$, and let $V$ be diverse. This means that an identity of the form $x_{1} \ldots x_{k}=w$ with $|w|>k=n_{D}(V)$ is fulfilled in $V$. Using Propositions 23 and 24 and Lemmas 2 and 3, we see that the semigroups $S L_{k}, S L_{k}^{*}, N_{k}$, and $C N_{k}$ do not belong to $V$, and, therefore, $\mathfrak{A}_{V}$ and $\mathfrak{N}_{V}$ are finite sets. Let $\left\{V_{i}\right\}_{1 \leq i \leq m}$ be a family of pairwise noncomparable subvarieties of $V$. By Theorem 3, each of the Rees-Sushkevich varieties $V_{i}$ can be decomposed into the sum of a radical variety $\mathfrak{R}_{i}$ and a semisimple variety $W_{i}$ such that

$$
\mathfrak{R}_{i}=\operatorname{Var}\left(\mathfrak{N}_{V_{i}}\right), \quad W_{i}=W_{i}^{\prime}+\operatorname{Var}\left(\mathfrak{A}_{W_{i}}\right) .
$$

Since $\mathfrak{A}_{W_{i}} \subset \mathfrak{A}_{V}$, the set $\left\{\mathfrak{A}_{W_{i}} \mid 1 \leq i \leq m\right\}$ has at most $d_{1}=2^{\left|\mathfrak{A}_{V}\right|}$ elements. Similarly, since $\mathfrak{N}_{V_{i}} \subset \mathfrak{N}_{V}$, Lemma 25] shows that the set $\left\{\mathfrak{N}_{V_{i}} \mid 1 \leq i \leq m\right\}$ has at most $d_{2}=2^{\left|\mathfrak{N}_{V}\right|}$ elements. At the same time, $\left\{W_{i}^{\prime}\right\}_{1 \leq i \leq m}$ is contained in the variety $V^{\prime}$ of width $d$. Therefore, $m \leq d \cdot d_{1} \cdot d_{2}$. Thus, the width of $V$ is finite and does not exceed $d \cdot d_{1} \cdot d_{2}$.

Proof of Corollary 11, Let $V$ be a Rees-Sushkevich variety. We must prove that the following statements are equivalent.

(1) The interval $\left[V^{\prime}, V\right]$ is finite.

(2) The interval $\left[V^{\prime}, V\right]$ satisfies the maximality condition.

(3) $V$ satisfies one of the following conditions: $A_{2} \in V, B_{2}, M_{p} \in V$ for a prime $p$, or $B_{2} \in V$ and $V$ is a $\lambda^{*}$ - and a $\rho^{*}$-variety.

The implication $(1) \Rightarrow(2)$ is obvious. 
$(2) \Rightarrow(3)$. Suppose that the interval $\left[V^{\prime}, V\right]$ satisfies the maximality condition and $A_{2} \notin V$. We consider all possible cases.

a) If $B_{2} \notin V$, then all completely 0-simple semigroups in $V$ are completely simple, possibly, with zero adjoined. Therefore, the identity $x=x^{n+1}$ is fulfilled in the variety $V^{\prime}=\operatorname{Var}\left(C S^{0} V\right)$. It follows that $C N_{k} \notin V^{\prime}$.

If $C N_{k} \in V$ for all $k$, then, in contradiction with (2), the varieties $W_{k}=\operatorname{Var}\left(C S^{0} V\right)+$ $\operatorname{Var}\left(C N_{k}\right)$ in the interval $\left[V^{\prime}, V\right]$ form a strictly increasing infinite chain. Indeed, the identity $x_{1} x_{2} \ldots x_{k+1}=\left(x_{1} x_{2} \ldots x_{k+1}\right)^{n+1}$ is fulfilled in $W_{k}$, because this identity is fulfilled in $C N_{k}$ and in $\operatorname{Var}\left(C S^{0} V\right)$. However, $C N_{k+1} \notin W_{k}$ by Lemma 3 Therefore, $W_{k}$ is a proper subvariety of $W_{k+1}$. Consequently, $C N_{k} \notin V$ for some $k$. By Lemma 3 , an identity of the form $x_{1} x_{2} x_{3} \ldots x_{k}=w$, where $|w|>k$, is fulfilled in $V$, which means that $V$ is a diverse variety.

b) Let $B_{2} \in V$, and let $M_{p} \notin V$ for all prime $p$. This means that all completely 0 -simple semigroups in $V$ are orthodox. Hence, the identity $a x^{2 n} y^{2 n} b=a y^{2 n} x^{2 n} b$ is fulfilled in $V^{\prime}=\operatorname{Var}\left(C S^{0} V\right)$.

If $S L_{k} \in V$ for all $k$, then, contrary to (2), the varieties $V_{k}=\operatorname{Var}\left(C S^{0} V\right)+\operatorname{Var}\left(S L_{k}\right)$ belong to the interval $\left[V^{\prime}, V\right]$ and form a strictly increasing infinite chain. Indeed, the identity

$$
q_{l, k}: x_{1} x_{2} \ldots x_{k+1} x^{2 n} y^{2 n} x^{2 n} y^{2 n}=x_{1} x_{2} \ldots x_{k+1} y^{2 n} x^{2 n} y^{2 n}
$$

is fulfilled in $V_{k}$, because this identity is fulfilled in every orthodox completely 0-simple semigroup and also in $S L_{k}$ by Lemma 16. However, $S L_{k+1} \notin V_{k}$ by Proposition 23. so that $V_{k}$ is a proper subvariety of $V_{k+1}$. Consequently, $S L_{k} \notin V$ for some $k$. By Proposition 23, one of the identities $q_{l, k}$ or $q_{l, k}^{*}: x_{1} x_{2} \ldots x_{k} y^{2}=x_{1} x_{2} \ldots x_{k}^{m+1} y^{2}$ must be fulfilled in $V$. However, this identity is not fulfilled in $B_{2} \in V$. Therefore, the identity $q_{l, k}$ is fulfilled in $V$. This means that $V$ is a $\lambda^{*}$-variety. Similarly, we can prove that $V$ is a $\rho^{*}$-variety.

$(3) \Rightarrow(1)$. We assume that (3) is fulfilled and consider all possible cases.

a) Let $V$ be a diverse variety. Then $n_{S L}, n_{R L} \leq n_{S L^{*}}$, and $n_{R S^{*}} \leq n_{C N}(V)<\infty$, whence $\mathfrak{A}_{V}$ is finite. On the other hand, the set of radical subvarieties of $V$ is finite because $V$ is diverse. By Theorem 1 , every variety $V^{*}$ in $\left[V^{\prime}, V\right]$ can be represented as the sum

$$
V^{*}=\operatorname{Var}\left(\mathfrak{N}_{V^{*}}\right)+\operatorname{Var}\left(C S^{0}(V)\right)+\operatorname{Var}\left(\mathfrak{A}_{V^{*}} \backslash \operatorname{Var}\left(C S^{0} V\right)\right) .
$$

Thus, the number of such subvarieties is finite.

b) $A_{2} \in V$. By the exactness criterion, we have $V^{\prime}=V$, and (1) becomes obvious.

c) $V$ is not a diverse variety, $A_{2} \notin V, B_{2} \in V$, and $M_{p} \in V$ for a prime $p$. Then all semigroups $S L_{k}$ are contained in $\operatorname{Var}\left(C S^{0} V\right)$. Indeed, assuming the contrary, we use Proposition 23 to show that there is $k$ such that one of the identities $q_{l, k}$ or $q_{l, k}^{*}$ is fulfilled in $\operatorname{Var}\left(C S^{0} V\right)$. However, the former is not fulfilled in $M_{p}$ and the latter is not fulfilled in $B_{2}$. Similarly, we can prove that all semigroups $S R_{k}$ are contained in $\operatorname{Var}\left(C S^{0} V\right)$. By Lemma 32, $\left[x^{2}=0, x y x=0\right] \subset \operatorname{Var}\left(B_{0}\right) \subset \operatorname{Var}\left(B_{2}\right)$. It follows that $N_{k}, C N_{k} \in \operatorname{Var}\left(B_{0}\right) \subset \operatorname{Var}\left(B_{2}\right)$ for all $k$. Consequently, the set $\mathfrak{A}_{V} \backslash \operatorname{Var}\left(C S^{0} V\right)$ is finite.

By Theorem 1, every variety $V^{*}$ in $\left[V^{\prime}, V\right]$ can be represented as the sum

$$
V^{*}=\operatorname{Var}\left(\mathfrak{N}_{V^{*}}\right)+\operatorname{Var}\left(C S^{0}\left(V^{*}\right)\right)+\operatorname{Var}\left(\mathfrak{A}_{V^{*}} \backslash \operatorname{Var}\left(C S^{0} V\right)\right) .
$$

As was mentioned above,

$$
\operatorname{Var}\left(\mathfrak{N}_{V^{*}}\right) \subset\left[x^{2}=0, x y x=0\right] \subset \operatorname{Var}\left(B_{2}\right) \subset \operatorname{Var}\left(C S^{0} V\right) .
$$

Therefore, $V^{*}=\operatorname{Var}\left(C S^{0}\left(V^{*}\right)\right)+\operatorname{Var}\left(\mathfrak{A}_{V^{*}} \backslash \operatorname{Var}\left(C S^{0} V\right)\right)$. Since $V^{\prime} \subset V^{*} \subset V$, we have $V^{\prime}=\left(V^{\prime}\right)^{\prime} \subset\left(V^{*}\right)^{\prime} \subset V^{\prime}$. Therefore,

$$
\operatorname{Var}\left(C S^{0}\left(V^{*}\right)\right)=\left(V^{*}\right)^{\prime}=V^{\prime}=\operatorname{Var}\left(C S^{0}(V)\right)
$$


whence $V^{*}=\operatorname{Var}\left(C S^{0}(V)\right)+\operatorname{Var}\left(\mathfrak{A}_{V^{*}} \backslash \operatorname{Var}\left(C S^{0} V\right)\right)$. However, $\mathfrak{A}_{V^{*}} \subset \mathfrak{A}_{V}$. It was proved above that the set $\mathfrak{A}_{V} \backslash \operatorname{Var}\left(C S^{0} V\right)$ is finite. Consequently, there are only finitely many subvarieties in the interval $\left[V^{\prime}, V\right]$.

d) Let $V$ be a nondiverse variety, and let $B_{2} \in V, A_{2} \notin V$, and $M_{p} \notin V$ for all $p$. Condition (3) implies that $V$ is a $\lambda^{*}$ - and a $\rho^{*}$-variety. By Proposition 22 the semigroups $S L_{k}$ and $S R_{k}$ do not belong to $V$, so that the set $\operatorname{Var}\left(\mathfrak{A}_{V} \backslash \operatorname{Var}\left(C S^{0} V\right)\right)$ is finite. Now, repeating the argument of item c), we conclude that the interval $\left[V^{\prime}, V\right]$ is finite.

Proof of Corollary 12, Let $V$ be a Rees-Sushkevich variety. We must prove that the following conditions are equivalent:

(1) $V$ is generated by a finite semigroup and a completely 0 -simple semigroup;

(2) if $V$ is a $\lambda$-variety, then $V$ is a $\lambda^{*}$-variety; if $V$ is a $\rho$-variety, then $V$ is a $\rho^{*}$-variety; if $B_{0} \notin V$, then $V$ is a diverse variety.

$(1) \Rightarrow(2)$. Let $V=\operatorname{Var}\left(C S^{0} V\right)+\operatorname{Var}(S)$, where $S$ is a finite semigroup. If $V$ is a $\lambda$-variety, then, by Proposition 8 , the identity

$$
e q B_{l, n}: z^{2 n} t x^{2 n} y^{2 n} x^{2 n} y^{2 n}=z^{2 n} t y^{2 n} x^{2 n} y^{2 n}
$$

is fulfilled in $V$ (and so also in $S$ ).

Let $m=|S|$. Then we have $X Y=X g Y$, where $X=x_{1} \ldots x_{k}$ and $Y=x_{k+1} \ldots x_{m}$, for arbitrary $x_{1}, x_{2}, \ldots, x_{m}$ in $S$, some $k<m$, and some idempotent $g$. It follows that

$$
\begin{aligned}
X Y x^{2 n} y^{2 n} x^{2 n} y^{2 n} & =X g Y x^{2 n} y^{2 n} x^{2 n} y^{2 n} \\
\stackrel{e q B_{l, n}}{=} & X g Y y^{2 n} x^{2 n} y^{2 n}=X Y y^{2 n} x^{2 n} y^{2 n} .
\end{aligned}
$$

Thus, the identity

$$
q_{l, m}: x_{1} \ldots x_{m} x^{2 n} y^{2 n} x^{2 n} y^{2 n}=x_{1} \ldots x_{m} y^{2 n} x^{2 n} y^{2 n}
$$

is fulfilled in $S$.

The identity $e q B_{l, n}$ is fulfilled in $V$, and, in particular, in all completely 0-simple semigroups in $V$. In the class of completely 0 -simple semigroups, the identity $q_{l, m}$ follows from $e q B_{l, n}$; therefore, the identity $q_{l, m}$ is fulfilled in $\operatorname{Var}\left(C S^{0} V\right)$. Consequently, the identity $q_{l, m}$ is fulfilled in $V=\operatorname{Var}\left(C S^{0} V\right)+\operatorname{Var}(S)$. This means that $V$ is a $\lambda^{*}$-variety. Similarly, we can prove that if $V$ is a $\rho$-variety, then $V$ is a $\rho^{*}$-variety.

However, if $B_{0} \notin V$, then the variety $V$ is diverse by Proposition 40 .

$(2) \Rightarrow(1)$. Suppose condition (2) is satisfied in $V$ and $B_{0} \in V$. By Lemma 25] we have

$$
\mathfrak{N}_{V} \subset\left[x^{2}=x y x=0\right] \subset \operatorname{Var}\left(B_{0}\right) \subset \operatorname{Var}\left(C S^{0} V\right) .
$$

Therefore, by Theorem 1, we obtain

$$
\begin{aligned}
V & =\operatorname{Var}\left(C S^{0} V\right)+\operatorname{Var}\left(\mathfrak{N}_{V}\right)+\operatorname{Var}\left(\mathfrak{A}_{V}\right) \\
& =\operatorname{Var}\left(C S^{0} V\right)+\operatorname{Var}\left(B_{0}\right)+\operatorname{Var}\left(\mathfrak{A}_{V} \backslash\left(\operatorname{Var}\left(C S^{0} V\right)+\operatorname{Var}\left(B_{0}\right)\right)\right) .
\end{aligned}
$$

It remains to prove that the set $\mathfrak{A}_{V} \backslash\left(\operatorname{Var}\left(C S^{0} V\right)+\operatorname{Var}\left(B_{0}\right)\right)$ is finite. The identities

$$
q_{l, k}^{*}: x_{1} x_{2} \ldots x_{k} y^{2}=x_{1} x_{2} \ldots x_{k}^{n+1} y^{2}, \quad q_{r, k}^{*}: y^{2} x_{1} x_{2} \ldots x_{k}=y^{2} x_{1}^{n+1} x_{2} \ldots x_{k}
$$

are not fulfilled in $B_{0}$. Therefore, all semigroups $S L_{k}^{*}, S R_{k}^{*}$ belong to $\operatorname{Var}\left(B_{0}\right)$ by Proposition 24. Since $B_{0} \in V$, Lemma 32 shows that

$$
N_{k}, C N_{k} \in\left[x^{2}=x y x=0\right] \subset \operatorname{Var}\left(B_{0}\right) \subset \operatorname{Var}\left(C S^{0} V\right)
$$

for all $k$. It remains to treat the semigroups $S L_{k}$ and $S R_{k}$. If $B_{l} \notin \operatorname{Var}\left(C S^{0} V\right)$, then $V$ is a $\lambda$-variety by Proposition 20. Therefore, condition (2) implies that $V$ is also a $\lambda^{*}$-variety. Now, Proposition 22 implies that none of the semigroups $S L_{k}$ belong to $V$. 
Let $B_{l} \in \operatorname{Var}\left(C S^{0} V\right)$. Using Lemma 14 and Proposition [6, we conclude that, for all $k$, the identities

$$
\begin{aligned}
& q_{l, k}: x_{1} x_{2} \ldots x_{k} x^{2 n} y^{2 n} x^{2 n} y^{2 n}=x_{1} x_{2} \ldots x_{k} y^{2 n} x^{2 n} y^{2 n}, \\
& q_{l, k}^{*}: x_{1} x_{2} \ldots x_{k} y^{2}=x_{1} x_{2} \ldots x_{k}^{m+1} y^{2}
\end{aligned}
$$

are not fulfilled, respectively, in the semigroups $B_{l}$ and $B_{0}$, and, therefore, also in the variety $\operatorname{Var}\left(C S^{0} V\right)$. Thus, by Proposition 23, all semigroups $S L_{k}$ are contained in $\operatorname{Var}\left(C S^{0} V\right)$. In the same way we can prove that the semigroups $S R_{k}$ either do not belong to $V$ or are contained in $\operatorname{Var}\left(C S^{0} V\right)$. Thus, the set $\mathfrak{A}_{V} \backslash\left(\operatorname{Var}\left(C S^{0} V\right)+\operatorname{Var}\left(B_{0}\right)\right)$ is finite, which implies the inclusion $(2) \Rightarrow(1)$ in the case where $B_{0} \in V$.

If $B_{0} \notin V$, then the variety $V$ is diverse by condition (2). Then Lemmas 2 and 3 and the definitions show that the sets $\mathfrak{N}_{V}$ and $\mathfrak{A}_{V}$ are finite. Therefore, the variety

$$
V=\operatorname{Var}\left(C S^{0} V\right)+\operatorname{Var}\left(\mathfrak{N}_{V}\right)+\operatorname{Var}\left(\mathfrak{R}_{V}\right)
$$

is generated by a completely 0 -simple semigroup and a finite semigroup.

Proof of Corollary 13 ( A criterion for finite generation). We must prove that a ReesSushkevich variety $V$ is generated by a finite semigroup if and only if its derivative $V^{\prime}$ is generated by a finite semigroup and conditions $(C F 1),(C F 2)$, and $(C F 3)$ of Corollary 12 are fulfilled. The "if" part follows immediately from Corollary 12 , We prove the "only if" part. Let $V$ be generated by a finite semigroup $S$. By Proposition 41, the variety $V^{\prime}$ is generated by a finite semigroup (e.g., by the 0 -direct product of all completely 0 -simple principal factors of the semigroup $S$ ). Then Corollary 12 shows that conditions $(C F 1)$, $(C F 2)$, and $(C F 3)$ are fulfilled.

Proof of Corollary 14. From Corollary 11, we see that a Rees-Sushkevich variety $V$ satisfies the maximality condition if and only if its derivative has the same property and the variety $V$ itself is diverse. This is exactly the claim of Corollary 14

Proof of Corollary 15. This corollary says that, in the lattice of subvarieties of $R S_{n}$, the unions and covers of varieties satisfying the maximality condition also satisfy the maximality condition. This follows from Corollaries 11 and 14 and also from the corresponding assertion for the lattice of subvarieties of the variety of the completely regular semigroups $\left[x=x^{n+1}\right.$ ( see [1]). It suffices to observe that $B_{2}$ is not contained in the diverse varieties $V \subset R S_{n}$ (because the required identity is not fulfilled in the semigroup $B_{0} \subset B_{2}$ by Proposition 6 ). Therefore, all completely 0 -simple semigroups in $V$ are completely simple, possibly, with zero adjoined, and, thus, the identity $x=x^{n+1}$ is fulfilled in $V^{\prime}$.

This corollary gives an answer to the question 10.2 in [1] for Rees-Sushkevich varieties.

Proof of Corollary 16 (A criterion for being small (Cross)). We claim that a Rees-Sushkevich variety $V$ is small (Cross) if and only if the derivative variety $V^{\prime}$ has the same property and the variety $V$ itself is diverse. This follows from Corollaries 2, 4, 11, and 13. In particular, a subvariety of $R S_{2}$ is small (Cross) if and only if it is diverse. Indeed, it suffices to observe that the groups of exponent 2 are Abelian, and the results of Mashevitskil and Fortunatov [5] imply that the varieties of periodic completely simple semigroups over Abelian groups are Cross. 


\section{$\S 7$. Algorithmic questions (Proofs)}

Proof of Theorem 6. A linear algorithm (in the sum of the lengths of the basis identities) for calculating the rank of a variety defined by a finite set of identities can easily be constructed with the help of Lemmas 14,17, 2, and 3 and Propositions 7 and 6. We illustrate this with an example. Let

$$
V=\left[x^{2}=x^{5}, x y x=x y x y x, x y x z x=x z x y z, a x y z t z=\text { axytzt } z\right] .
$$

By Propositions 7 and [6, we have $A_{0}, B_{0} \in V$. From Lemmas 2 and 3 , it follows that $N_{k}$ and $C N_{k} \in V$ for all $k$. By Lemma 14, we have $B_{l} \notin V$ and $B_{r} \in V$, and Lemma 15] shows that $A_{l}^{*}, A_{r} \in V$. From Lemma 16, it follows that $S L_{2} \in V, S L_{3} \notin V$, and $S R_{k} \in V$ for all $k$, and, by Lemma 17, we obtain $S L_{k}^{*}, S R_{k}^{*} \in V$ for all $k$. Thus,

$$
\operatorname{Rank}(V)=(1,1,0,1,1,1, \infty, \infty, 2, \infty, \infty, \infty) .
$$

Proof of Theorem 17. We must prove that the equational theory of a Rees-Sushkevich variety $V$ defined by a finite semigroup or a finite set of identities is decidable (polynomially decidable) if and only if the derivative variety $V^{\prime}$ has the same property. The "only if" part follows from Proposition 42, We prove the converse. Suppose that the derivative variety $V^{\prime}$ have a decidable (polynomially decidable) equational theory. An identity $u=v$ is fulfilled in $V$ if and only if $V[u=v]=V$. By Theorem 1, we have $V=\operatorname{Var}\left(C S^{0} V, \mathfrak{A}_{V}, \mathfrak{N}_{V}\right)$ and

$$
V[u=v]=\operatorname{Var}\left(C S^{0} V[u=v], \mathfrak{A}_{V[u=v]}, \mathfrak{N}_{V[u=v]}\right) .
$$

This means that $V[u=v]=V$ if and only if $V^{\prime}[u=v]=V^{\prime}, \operatorname{Rank} V[u=v]=\operatorname{Rank} V$, and $\mathfrak{N}_{V[u=v]}=\mathfrak{N}_{V}$. The first relation is valid if and only if the identity $u=v$ is fulfilled in $V^{\prime}$ and can be verified (can be verified in polynomial time) because, by assumption, $V^{\prime}$ has a decidable (polynomially decidable) equational theory.

If $V$ has a finite basis of identities, then, by Theorem [6, equality of the ranks of the varieties $V$ and $V[u=v]$ can be verified in polynomial time in the length of the identity $u=v$. It is a little more complicated to prove this if $V$ is generated by a finite semigroup $S$ of period $n$. By Theorem 4 , the variety $V$ has a basis $T$ of identities contained in the union of the sets $E q R S_{n}, E q N$, and Eqreg. We denote by $T_{1}, T_{2}$, and $T_{3}$ the intersection of $T$ with $E q R S_{n}, E q N$, and Eqreg, respectively. By the definitions, the set of identities $T_{1}$, as every set of identities in $E q R S_{n}$, has a finite basis $T_{1}^{*}$ and this basis can be found effectively by the Cayley table of the finite semigroup $S$. Indeed, the verification of the fact that identities in $E q R S_{n}$ depending on a parameter are fulfilled in a finite semigroup $S$ of $m$ elements reduces to the verification of identities for which the value of the parameter is bounded by the number $m$. For example, if $k>m$, then the identity $q_{l, k}: x_{1} x_{2} \ldots x_{k} x^{2 n} y^{2 n} x^{2 n} y^{2 n}=x_{1} x_{2} \ldots x_{k} y^{2 n} x^{2 n} y^{2 n}$ is fulfilled in a semigroup $S$ if and only if the identity $q_{l, m}$ is fulfilled in $S$ (because if $S$ is a semigroup of $m$ elements, then $\left.S^{m}=S^{m+1}=\cdots\right)$. By Proposition 34, the set $T_{2}$ of permutation identities has a finite basis $T_{2}^{*}$, which can be found effectively by the Cayley table of $S$ (see [14]).

In the proof of Theorem 4 we verified, in particular, that if $V \subset R S_{n}$ is a ReesSushkevich variety, then the set $\mathfrak{A}_{V}$ is uniquely determined by the identities in $E q R S_{n}$ and $E q N$ fulfilled in $V$ (see Remark [6). Since the identities of the indicated form for $V$ are the same as for the finitely based variety

$$
V^{\#}=\left[\mathrm{I}_{n}, \mathrm{II}_{n}, \mathrm{III}_{n}, T_{1}, T_{2}\right]=\left[\mathrm{I}_{n}, \mathrm{II}_{n}, \mathrm{III}_{n}, T_{1}^{*}, T_{2}^{*}\right],
$$

we see that $\mathfrak{A}_{V}=\mathfrak{A}_{V \#}$. We also note that

$$
\mathfrak{A}_{V[u=v]}=\mathfrak{A}_{V} \cap \mathfrak{A}_{[u=v]}=\mathfrak{A}_{V \#} \cap \mathfrak{A}_{[u=v]} .
$$


Therefore, $\operatorname{Rank} V[u=v]=\inf \left(\operatorname{Rank} V^{\#}, \operatorname{Rank}[u=v]\right)$, and, by Theorem 6 , the relation Rank $V[u=v]=\operatorname{Rank} V$ can be verified in time polynomial in the length of the identity.

To verify the last relation $\mathfrak{N}_{V[u=v]}=\mathfrak{N}_{V}$, we consider all possible cases.

1. If $u=v$ is not a permutation or a diverse identity, then, by the definition, we have $\mathfrak{N}_{V[u=v]}=\mathfrak{N}_{V}$.

2. If the identity $u=v$ is diverse, i.e., has the form $x_{1} \ldots x_{s}=w,|w|>s$, then $\mathfrak{N}_{V[u=v]}=\mathfrak{N}_{V}$ is equivalent to $s \geq n_{D}(V)$. However, if a variety $V$ is defined by a finite set $X$ of identities, then its diversity degree $n_{D}(V)$ is equal to the length of the shortest singular word among the words $u^{\prime}$ and $v^{\prime}$ such that the identity $u^{\prime}=v^{\prime}$ belongs to $X$, is not permutation, and is not a tautology (i.e., $u^{\prime} \not \equiv v^{\prime}$ ). Similarly, if $V=[S]$, then the diversity degree $n_{D}(V)$ of $V$ is equal to the length of the shortest singular word among the words $u^{\prime}$ and $v^{\prime}$ such that the identity $u^{\prime}=v^{\prime}$ belongs to $T_{1}^{*}$.

3. If $u=v$ is a permutation identity, then $\mathfrak{N}_{V[u=v]}=\mathfrak{N}_{V}$ if and only if the identity $u=v$ is a consequence of the permutation identities $u^{\prime}=v^{\prime}$ of length less than the diversity degree $n_{D}(V)$ that belong to the finite basis $X$ of identities for the variety $V$ or to the set $T_{2}^{*}$ (if $V=[S]$ ). It is easy to check (see [14]) that the problem of deciding whether or not a permutation identity $u=v$ is a consequence of a fixed finite set of permutation identities has complexity linear in the length of the identity $u=v$.

Theorem 7 is proved completely.

Proof of Corollary 17. Theorem 7 and the fact that the equational theory of finite Rees matrix semigroups over a one-element group is polynomially decidable (see [19]) imply that every Rees-Sushkevich variety in $R S_{1}$ defined by a finite semigroup or by a finite set of identities has a polynomially decidable equational theory (it suffices to observe that, by Corollary [3, every exact variety $V \subset R S_{1}$ is generated by a finite completely 0-simple semigroup). This is exactly the claim of the corollary.

Remark 7. Examples constructed in the papers [18, 3] show that the statement proved above is valid neither for the variety $R S_{2}$ nor for the class of finite combinatorial semigroups.

Proof of Theorem 8. This theorem says that there is an algorithm of polynomial complexity allowing one to decide whether or not a periodic variety of semigroups defined by a finite set of identities or by a finite semigroup is generated by completely 0 -simple semigroups. First, let $V$ be a periodic variety of semigroups of period $n$ defined by a finite set of identities. In the paper [20], the author constructed a family of 13 finite indicator semigroups the absence of which in a given variety of semigroups is a necessary and sufficient condition for $V \subset R S_{n}$. In the recent paper [17, Bakulin proved that the verification of identities in indicator semigroups has polynomial complexity. By Proposition 17 and Lemma 16] the verification of identities in the semigroups $A_{0}, S L_{1}$, and $S R_{1}$ has linear complexity. The description of the identities in the semigroups $A_{2}, B_{2}$, and $M_{p}$ is well known. The verification of identities in these semigroups can be performed in polynomial time [19, 5]. By Rees's theorem, a periodic variety $V$ is generated by 0 -simple semigroups if and only if $V$ is generated by completely 0 -simple semigroups. Using Corollary 1 (the exactness criterion), we conclude that the verification whether or not a variety $V$ is exact has polynomial complexity.

Now, let $V=V[S]$ be a variety of semigroups generated by a finite semigroup $S$ of $m$ elements. The inclusion $V \subset R S_{n}$ (for some $n$ ) holds true if and only if the identities $\mathrm{I}_{n}, \mathrm{II}_{n}$, and $\mathrm{III}_{n}$ are fulfilled in the semigroup $S$. For an arbitrary element $a$ of a finite semigroup of $m$ elements, there is an integer $k_{a} \leq m$ such that $a^{k_{a}}$ is an idempotent. In $S$, we define the unary operation $\bar{a}=a^{k_{a}}$. Observe that, for each element, the result of this operation can be obtained in at most $2 m$ steps. We also note that an identity of the 
form $\mathrm{I}_{n}$ is fulfilled in $S$ if and only if the identity $x^{2}=x^{2} \bar{x}$ is fulfilled in $S$. The latter identity can be verified in $O\left(\mathrm{~m}^{2}\right)$ steps. An identity of the form $\mathrm{II}_{n}$ is fulfilled in $S$ if and only if the identity $x y x=\overline{x y}(x y x)$ is valid, which can be verified in $O\left(m^{3}\right)$ steps. An identity of the form $\mathrm{III}_{n}$ is fulfilled in $S$ if and only if the identity $\overline{x h z}(x z)=(x z) \overline{x h z}$ is valid, which can be verified in $O\left(\mathrm{~m}^{4}\right)$ steps. Thus, there is an algorithm of polynomial complexity in the number of elements that decides whether or not a given semigroup is a Rees-Sushkevich semigroup.

Assume we have already established that $S \in R S_{n}$ for some $n$. By Proposition 37. the following properties are valid for Rees-Sushkevich varieties $V \subset R S_{n}$ :

1) $A_{2} \notin V \Leftrightarrow$ the identity $x^{2 n} y x^{2 n}=x^{2 n} y^{n+1} x^{2 n}$ is fulfilled in $V$;

2) $B_{2} \notin V \Leftrightarrow$ the identity $x y x=x y^{n+1} x$ is fulfilled in $V$;

3) $A_{0} \notin V \Leftrightarrow$ the identity $x^{2 n} y^{2 n}=\left(x^{2 n} y^{2 n}\right)^{n+1}$ is fulfilled in $V$;

4) $B_{0} \notin V \Leftrightarrow$ the identity $x^{2 n} y z^{2 n}=x^{2 n} y^{n+1} z^{2 n}$ is fulfilled in $V$;

5) $M_{p} \notin V$ for all $p \Leftrightarrow$ the identity $\left(x^{2 n} y^{2 n}\right)^{n+1}=\left(x^{2 n} y^{2 n}\right)^{n+2}$ is fulfilled in $V$;

6) $S L_{1} \notin V \wedge Y_{2} \in V \Leftrightarrow$ one of the identities

$$
x y^{2}=x^{n+1} y^{2} \quad \text { or } \quad x_{1} x^{2 n} y^{2 n} x^{2 n} y^{2 n}=x_{1} y^{2 n} x^{2 n} y^{2 n}
$$

is fulfilled in $V$ (see Proposition [23);

7) $S R_{1} \notin V \wedge Y_{2} \in V \Leftrightarrow$ one of the identities

$$
y^{2} x=y^{2} x^{n+1} \quad \text { or } \quad x^{2 n} y^{2 n} x^{2 n} y^{2 n} x_{1}=x^{2 n} y^{2 n} x^{2 n} x_{1}
$$

is fulfilled in $V$ (see Proposition 23).

The above-listed identities are fulfilled in a semigroup $S$ if and only if the corresponding identities with the unary operation $\bar{x}$ without parameter $n$ are fulfilled. For example, the identity $x^{2 n} y x^{2 n}=x^{2 n} y^{n+1} x^{2 n}$ is fulfilled in $S$ if and only if the identity $\bar{x} y \bar{x}=$ $\bar{x}(y \cdot \bar{y}) \bar{x}$ is fulfilled, which can be verified in $S$ in $m(m-1)(2 m+5)$ steps. These remarks and Corollary 1 (the exactness criterion) show that there is an algorithm of polynomial complexity in the number of elements that decides whether or not a given finite semigroup generates an exact variety. This is precisely the content of Theorem 8 for varieties generated by finite semigroups.

\section{REFERENCES}

[1] L. N. Shevrin, B. M. Vernikov, and M. V. Volkov, Lattices of semigroup varieties, Izv. Vyssh. Uchebn. Zaved. 2009, no. 3, 3-36; English transl., Russian Math. (Iz. VUZ) 53 (2009), no. 3, 1-28. MR 2581451 (2010m:20091)

[2] M. V. Sapir and E. V. Sukhanov, Varieties of periodic semigroups, Izv. Vyssh. Uchebn. Zaved. 1981, no. 4, 48-55; English transl. in Soviet Math. (Iz. VUZ) 25 (1981). MR0624862 (82j:20108)

[3] S. V. Pleshcheva and I. V. Verteshi, Complexity of the problem of identity verification in a certain finite 0-simple semigroup, Ural. Gos. Univ. Mat. Zap. 43 (2006), 72-102. (Russian)

[4] V. L. Murskiı̌, Some examples of varieties of semigroups, Mat. Zametki 3 (1968), no. 6, 663-670. (Russian) MR0231930 (38:256)

[5] G. I. Mashevitskil, On identities in varieties of completely simple semigroups over abelian groups, Modern Algebra, Leningrad. Ped. Inst., Leningrad, 1978, pp. 81-89. (Russian)

[6] M. V. Volkov, Bases of identities of Brandt semigroups, Ural. Gos. Univ. Mat. Zap. 14 (1985), no. 1, 38-42. (Russian). MR0867373 (88a:20070)

[7] A. H. Clifford and G. B. Preston, The algebraic theory of semigroups. Vols. I, II, Math. Surveys, No. 7, Amer. Math. Soc., Providence, RI, 1961, 1967. MR0132791 (24:A2627) MR0218472 $(36: 1558)$

[8] Sheila Oates and M. B. Powell, Identical relations in finite groups, J. Algebra 1 (1964), 11-39. MR0161904(28:5108)

[9] T. E. Hall, S. Kublanovskiǔ, S. Margolis, M. Sapir, and P. Trotter, Algorithmic problems for finite groups and finite 0-simple semigroups, J. Pure Appl. Algebra 119 (1997), 75-96. MR1456096 (98h:20101)

[10] E. W. H. Lee, Subvarieties of the variety generated by the five-element Brandt semigroup, Internat. J. Algebra Comput. 16 (2006), no. 2, 417-441. MR2228521 (2007c:20130) 
[11] - Combinatorial Rees - Sushkevich varieties are finitely based, Internat. J. Algebra Comput. 18 (2008), no. 5, 957-978. MR2440721 (2009k:20125)

[12] Combinatorial Rees-Sushkevich varieties that are Cross, finitely generated, or small, Bull. Austral. Math. Soc. 81 (2010), no. 1, 64-84. MR2584924(2011a:20147)

[13] S. A. Malyshev, Permutational varieties of semigroups whose lattice of subvarieties is finite, Modern Algebra, Leningrad. Ped. Inst., Leningrad, 1981, pp. 71-76. (Russian) MR0662692 (83j:20062)

[14] Gy. Pollák, On the consequences of permutation identities, Acta Sci. Math. (Szeged) 34 (1973), 323-338. MR0322084 (48:448)

[15] D. Rees, On semi-groups, Proc. Cambridge Philos. Soc. 36 (1940), 387-400. MR0002893 (2:127g)

[16] N. Reilly, Varieties generated by completely 0-simple semigroups, J. Austral. Math. Soc. 84 (2008), no. 3, 375-403. MR2453687 (2009i:20108)

[17] S. Bakulin, On identities of indicator Burnside semigroups, arXiv:1009.2337v1 [math.GR] .

[18] S. Seif, The Perkins semigroup has co-NP-complete term-equivalence problem, Internat. J. Algebra Comput. 15 (2005), no. 2, 317-326. MR2142086 (2005m:68090)

[19] S. Seif and Cs. Szabó, Computational complexity of checking identities in 0-simple semigroups and matrix semigroups over finite fields, Semigroup Forum 72 (2006), no. 2, 207-222. MR2216090 (2007a:20055)

[20] S. Kublanovsky, On the Rees-Sushkevich variety, arXiv:1009.2982v1 [math.GR] .

[21] _ Cyclically regular semigroups, arXiv:1103.3246v1 [math.GR] .

[22] P. M. Cohn, Universal Algebra, 2nd ed., Math. Appl., vol. 6, D. Reidel Publ. Co., Dordrecht, 1981. MR.0620952(82j:08001)

[23] B. M. Vernikov, Upper modular elements of the lattice of semigroup varieties, Algebra Universalis 59 (2008), 405-428. MR2470588 (2009i:20109)

TPO Severny Ochag, B. Konyushennaya 15, Office 30, St. Petersburg 191186, Russia E-mail address: stas1107@mail.ru

Received 13/DEC/2009

Translated by B. M. BEKKER 